University of Louisville

ThinkIR: The University of Louisville's Institutional Repository

Electronic Theses and Dissertations

$5-2021$

\title{
Determination of the biological functions of undefined domains of VEEV nsP2.
}

Andrew Michael Skidmore

University of Louisville

Follow this and additional works at: https://ir.library.louisville.edu/etd

Part of the Virology Commons

\section{Recommended Citation}

Skidmore, Andrew Michael, "Determination of the biological functions of undefined domains of VEEV nsP2." (2021). Electronic Theses and Dissertations. Paper 3648.

https://doi.org/10.18297/etd/3648

This Doctoral Dissertation is brought to you for free and open access by ThinkIR: The University of Louisville's Institutional Repository. It has been accepted for inclusion in Electronic Theses and Dissertations by an authorized administrator of ThinkIR: The University of Louisville's Institutional Repository. This title appears here courtesy of the author, who has retained all other copyrights. For more information, please contact thinkir@louisville.edu. 


\title{
DETERMINATION OF THE BIOLOGICAL FUNCTIONS OF UNDEFINED DOMAINS OF VEEV NSP2
}

\author{
By \\ Andrew Michael Skidmore \\ B.S., Bellarmine University, 2015 \\ M.S., University of Louisville, 2017

\begin{abstract}
A Dissertation
Submitted to the Faculty of the

School of Medicine of the University of Louisville

In Partial Fulfillment of the Requirements

For the Degree of
\end{abstract} \\ Doctor of Philosophy in Microbiology and Immunology \\ Department of Microbiology and Immunology \\ University of Louisville
}

May 2021 
Copyright 2021 by Andrew Skidmore

All rights reserved 



\title{
DETERMINATION OF THE BIOLOGICAL FUNCTIONS OF UNDEFINED DOMAINS OF VEEV NSP2
}

\author{
By \\ Andrew Michael Skidmore \\ B.S., Bellarmine University, 2015 \\ M.S., University of Louisville, 2017
}

A Dissertation Approved on

April 9, 2021

By the Following Dissertation Committee Members:

Donghoon Chung, PhD

(Dissertation Director)

Kevin Sokoloski, $\mathrm{PhD}$

Carolyn Casella, PhD

James Graham, PhD

Barbara Clark, PhD

Donghan Lee, PhD 


\section{DEDICATION}

This dissertation is dedicated to my parents who supported me the whole way, and made me believe that I could achieve anything if I persevered. To my sister and brother

in law who were always there to keep my sane. To my friends who commiserated and made this journey half party. And to the many baked good that were made and consumed to lessen my stress. 


\section{ACKNOWLEDGEMENTS}

I would first like to thank Dr. Chung, who really took a chance on me when he took me on as a student. I had no real research experience to speak of and you taught me all of my skills both at the bench and off. I greatly appreciate everything that have taught me about being a scientist and all which that entails.

I would like thank all of my committee members, who have been truly supportive of me every step of the way. You have each been instrumental in my scientific development and were valued experts when it came to assistance with experiments, and also when grad school at times became overwhelming.

To my lab mates, Eunjung Kim, Scott Adcock, Bryan Alejandro, and Brooke Armante I would just like to thank you all for making the lab a truly positive environment to be in. And for letting me talk entirely too much all the time. Brooke in particular was also instrumental in this dissertation, helping to generate parts of the data presented in the $4^{\text {th }}$ chapter.

I would like to thank all of the friends that I have made during graduate school. There are too many to list, but you all were really my support structure throughout this time and I would not have made it here without you. In particular I would like to thank Irina, Chris, Katlin, and Amanda. You all were always there to talk, complain, or commiserate and helped to ensure that I wasn't driven insane by grad school. 
I would also like to thank my friends who are currently still in graduate school, the other members of Louisvirology, Autumn, Claire, and Trey, and honorary virologists Will and Sarah. You guys are the best and you keep work bearable when the going gets tough. And you put up with me in all of annoying habits.

Finally I would like thank my family, who have been there every step of the way and supported me so much to allow me to pursue this degree. You all were always understanding of how busy I was and the time commitment it took. And you never asked me too often about when I would get done. Thank you so much for everything that you have provided me and the support that you have given. 


\section{ABSTRACT \\ DETERMINATION OF THE BIOLOGICAL FUNCTIONS OF UNDEFINED DOMAINS OF VEEV NSP2}

Andrew Michael Skidmore

April 9, 2021

Alphaviruses are positive sense, single strand, RNA viruses. These viruses occur on every populated continent. Alphaviruses are divided into two clades, the New-World and Old-World viruses. The New-World viruses include Eastern (EEEV), Western (WEEV), and Venezuelan equine encephalitis viruses (VEEV), and cause neuroinvasive disease. The Old-World viruses include Chikungunya (CHIKV) and Ross River viruses (RRV), and typically cause multijoint arthralgia. There are currently no approved antiviral therapeutics or vaccines for any alphavirus, making them a high priority for antiviral drug design and discovery. A benzamidine inhibitor (ML336) of VEEV was characterized, and determined to inhibit replication of VEEV RNA during infection of BHK-21 cells, a fibroblast model. This activity was due a loss of synthesis of new viral RNA. This compound had no effect on RNA synthesis in uninfected cells, making it a promising target for therapeutic development. The inhibitory activity of ML336 was highly specific for VEEV, having no effect on RNA synthesis of CHIKV. A potential interaction between ML336 and the VEEV nsPs was examined, but these results were inconclusive. ML336 and related compounds were used to generate resistant mutant VEEV. These isolates were sequenced and it was revealed that mutations were concentrated in a region of nsP2 of unknown function. Analysis of these mutant viruses revealed delayed growth, RNA synthesis, and translation of viral proteins in BHK cells. There was also a growth delay seen in SH-SY5Y cells, a model of neuronal infection. These findings indicate that this region of nsP2 is likely involved in RNA synthesis of VEEV, and shows promise as a target of antiviral drug development. 


\section{TABLE OF CONTENTS}

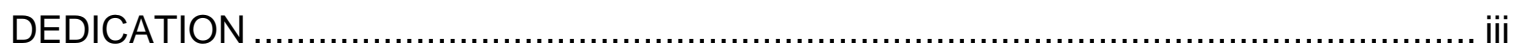

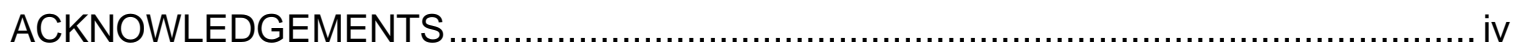

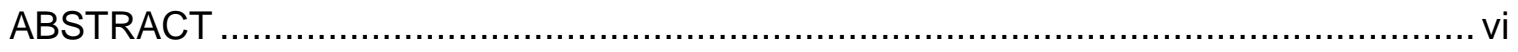

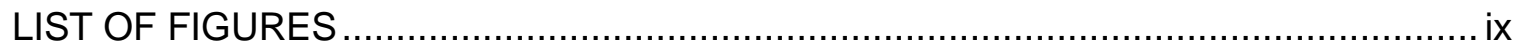

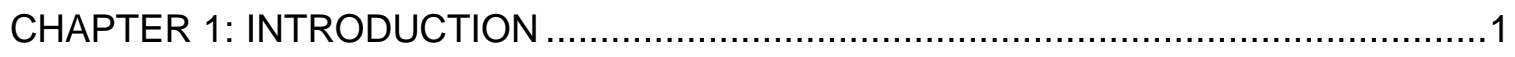

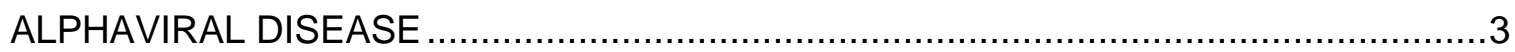

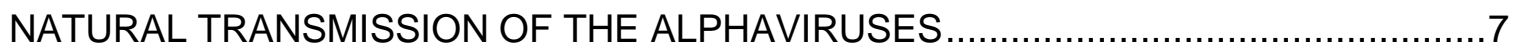

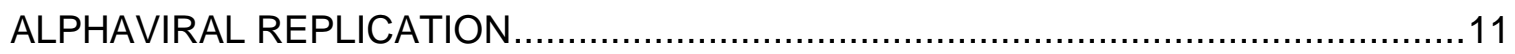

FUNCTIONS OF THE ALPHAVIRAL NONSTRUCTURAL PROTEINS ......................22

CURRENT STATE OF ALPHAVIRAL THERAPY DEVELOPMENT ...........................28

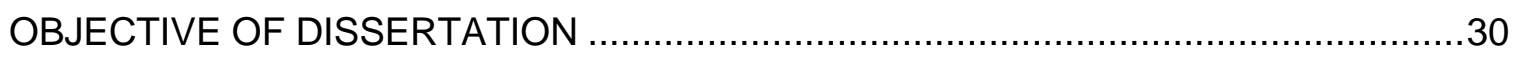

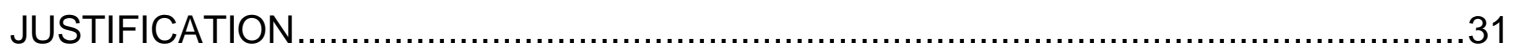

CHAPTER 2: BENZAMIDINE ML336 INHIBITS VEEV RNA SYNTHESIS ...................33

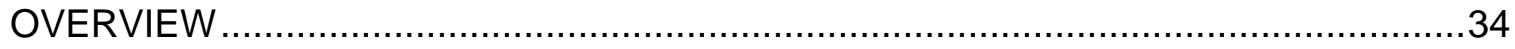

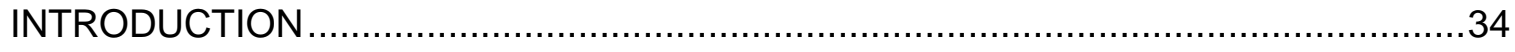

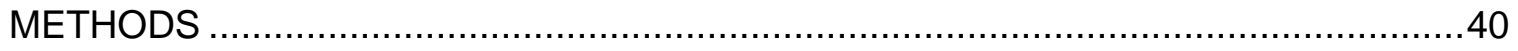

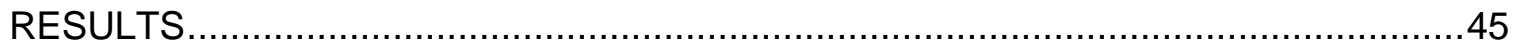

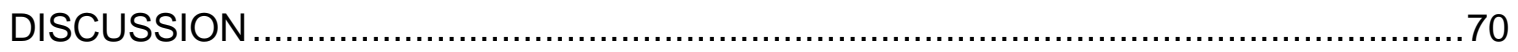

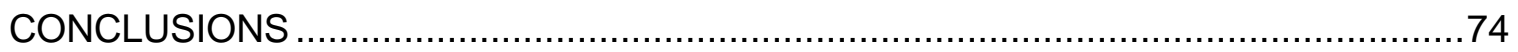

CHAPTER 3: ANALYSIS OF INTERACTIONS BETWEEN AMDIDINE COMPOUNDS

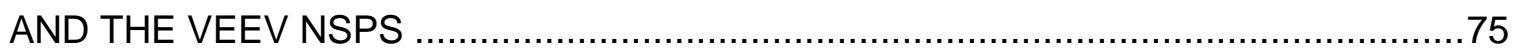

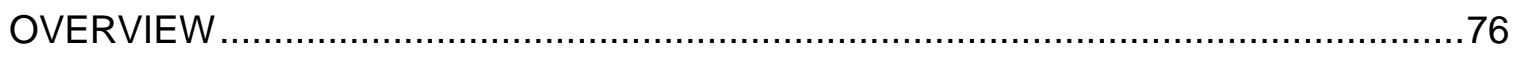

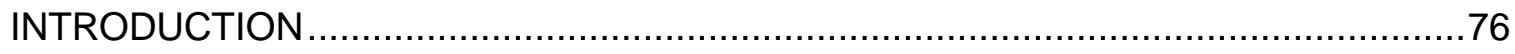




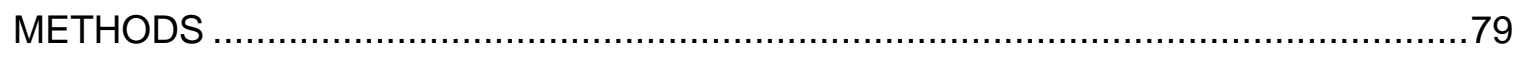

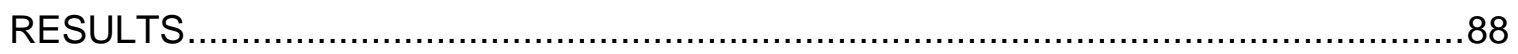

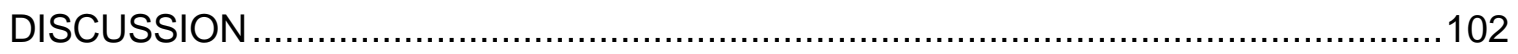

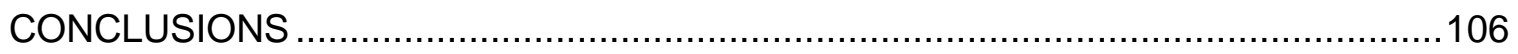

CHAPTER 4: THE USE OF AMIDINE COMPOUNDS TO CHARACTERIZE A DOMAIN

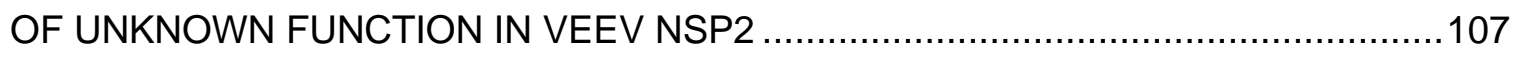

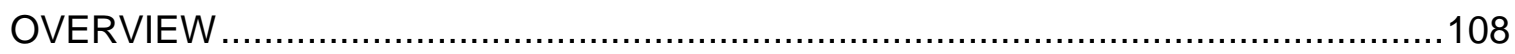

INTRODUCTION

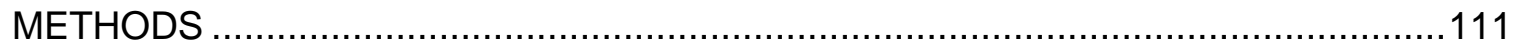

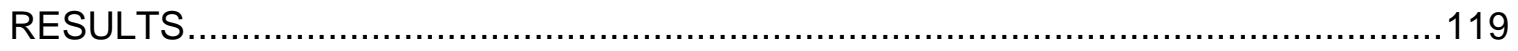

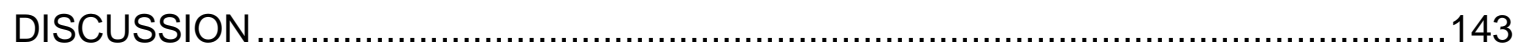

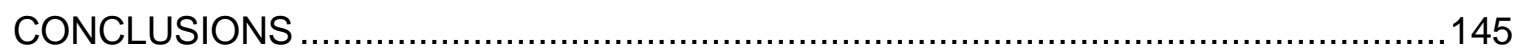

CHAPTER 5: DISCUSSION AND FUTURE DIRECTIONS .......................................146

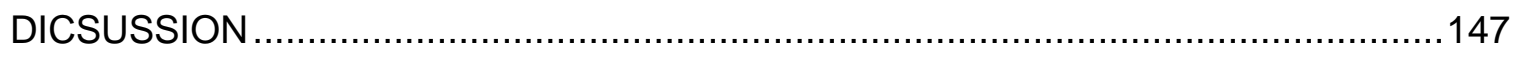

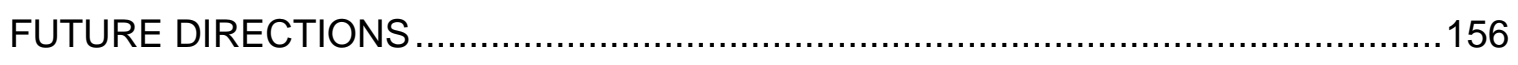

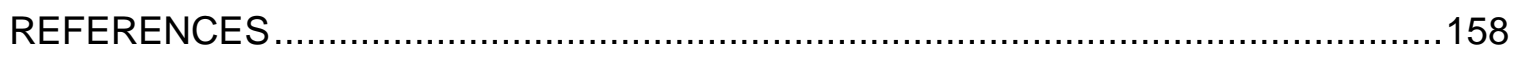

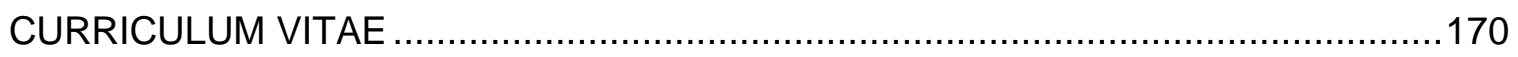


CHAPTER 1

LIST OF FIGURES

Figure 1. Pathology of alphaviruses..................................................................

Figure 2. Transmission cycle of alphaviruses ..........................................................

Figure 3. The genetic structure of VEEV .............................................................. 14

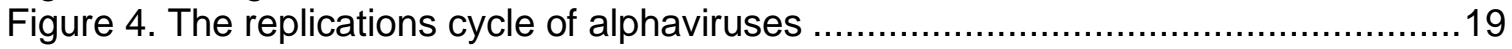

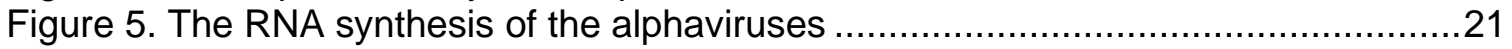

\section{CHAPTER 2}

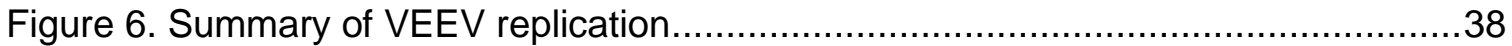

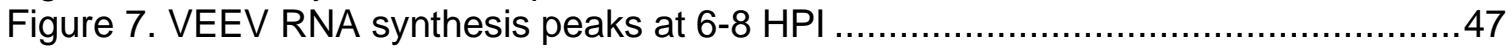

Figure 8. Compounds derived from ML336 also inhibit viral RNA synthesis .................50

Figure 9. The RNA synthesis inhibition of ML336 is highly specific and has no effect on

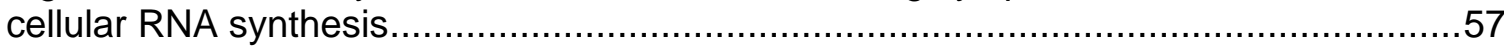

Figure 10. ML336 resistant mutations grant resistance to RNA synthesis inhibition .......60

Figure 11. ML336 inhibits all stages of VEEV RNA synthesis ...................................64

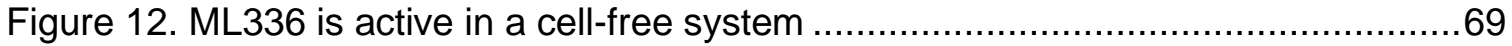

Figure 13. Homology model of VEEV nsP2 $\mathrm{N}$ terminal region ..................................72

\section{CHAPTER 3}

Figure 14. Ultracentrifugation did not successfully isolate the viral nsPs. 91

Figure 15. Use of Click-iT chemistry to identify viral proteins and compound/protein interactions.

Figure 16. Expression constructs used for ectopic synthesis of VEEV nsP2 ................99

Figure 17. The expression and purification of VEEV nsP2 in bacterial cells.....

\section{CHAPTER 4}

Figure 18. Single nucleotide polymorphisms of the selected viral isolates in N-nsP2 ...120 Figure 19. Maps of the full length genomes of the selected mutant viruses .................121

Figure 20. Mutant viral isolates show delayed growth in a fibroblast cell model ...........126

Figure 21. Mutant viral isolates have a delay in positive sense RNA synthesis ............130

Figure 22.Isolate 6_13_25 has a delay in negative sense RNA synthesis .................131

Figure 23. Relative expression of the subgenomic RNA compared to genomic RNA...132

Figure 24. Representative Western blot images examining viral protein expression ....135

Figure 25. Quantification of viral protein expression ............................................. 137

Figure 26. Compound resistant viruses have delayed growth in a neural cell model....141

\section{CHAPTER 5}

Figure 27. Working model of the effects of mutations in the N-terminal region of VEEV

nsP2. 
CHAPTER 1

INTRODUCTION 
Alphaviruses are positive sense, single stranded, RNA viruses in the family Togaviridae, which are classified as members of the domain Riboviria'. The alphaviruses currently encompass more than thirty members that infect a wide range of host and vector species, both terrestrial and aquatic. These viruses are widely dispersed geographically as well, with at least one alphavirus being present on every populated continent ${ }^{23-6}$. These viruses are currently emerging into naïve populations making them an important group of pathogens as there are currently no treatments or vaccines for alphaviral disease.

The geographic distribution of the alphaviruses has resulted in the historical classification of the viruses based on where they were initially isolated. The Old-World viruses were initially isolated in the Eastern hemisphere, primarily in Africa. The NewWorld viruses were isolated in the Americas, and have a more diverse native distribution, with members being found from southern Canada all the way to Argentina. These two clades are also regarded as having distinct symptoms in the host.

The Old-World viruses generally cause arthralgia and fever, with some also causing a rash ${ }^{7}$. There is some recent evidence that Old-World members may be able to cause encephalitis as well, with the adaptation of neurologically invasive Sindbis (SINV) virus in the lab, as well as its association with rare cases of viral encephalitis in Europe ${ }^{8-}$ 10. Neurological invasion is a common symptom of the New-World alphaviruses, with the three most prominent members of this clade: Eastern (EEEV), Western (WEEV), and Venezuelan Equine Encephalitis viruses (VEEV), demonstrating high levels of neurological pathogenicity ${ }^{7}$. Recent research has indicated that this division may be less stringent than previously thought, as many recently discovered New-World viruses do not cause detectable disease in humans or other host animals at all ${ }^{11}$. Additionally, 
several New-World viruses which may display pathogenicity that is more in line with that of the Old-World viruses, such as Mayaro virus ${ }^{2,12}$.

\section{Alphaviral Disease}

The following section will discuss the diseases that are caused by alphaviruses in the human population.

The Old-World virus of most concern is Chikungunya virus (CHIKV), which has recently expanded into naïve populations across Asia, southern Europe, and most dramatically in the Caribbean ${ }^{3-6}$. This has resulted in CHIKV becoming endemic in several regions where it previously had never achieved local transmission. The primary risk of these viruses is the sustained arthralgia that can last for months, with one study in Mexico indicating that over a third of confirmed CHIKV cases have arthralgia twelve months after acute disease ${ }^{13}$. Similar instances of long term pain have been seen in other family members as well, such as Ross River virus ${ }^{14}$.

The New-World alphaviruses generally cause more severe disease than the OldWorld viruses, however the three most common (EEEV, WEEV, VEEV) are noted for displaying a high rate of asymptomatic infection ${ }^{11}$. This asymptomatic infection rate does vary between the three viruses and in the two primary populations of interest, equids and humans ${ }^{11}$. Disease is also generally more severe in equid hosts than in humans ${ }^{7}$. Disease generally reduces in severity comparing EEEV to WEEV to VEEV, with EEEV having the highest reported rate of neurological involvement and lethality, VEEV having the least, and WEEV falling between the two ${ }^{11}$. However VEEV has historically caused the largest and largest number of outbreaks affecting both human and equid populations resulting in many thousands of human cases and equid deaths ${ }^{11,15}$. 
EEEV is the most severe of the New World alphaviruses with case fatality rates in some cases estimated to be above $60 \%{ }^{16}$ and infection results in death or permanent neurological sequelae in a large majority of symptomatic cases ${ }^{17}$. The virus remains uncommon in the human population, with only in a handful of reported cases every year in the United States ${ }^{18}$. There has recently been an uptick in cases of EEEV reported to the $C D C$, with almost 40 cases reported in 2019, a more than fivefold increase from $2018^{18}$.

WEEV is of moderate pathogenicity, with a case fatality and rate of neurological involvement between that of EEEV and VEEV. While the virus has caused instances of severe human disease, its occurrence in the human population is quite low $^{11}$ and has been declining for many years since its initial discovery in the 1930s. In fact, since 1964 there have been less than 700 cases of WEEV reported in the United States, averaging out to less than 12 cases a year ${ }^{19}$. And there has not been a reported case of human WEEV disease in the United States since $1998^{20}$.

VEEV is the least severe of the encephalitic alphaviruses. Conversely it is also the virus of most concern for two reasons. First, unlike the other encephalitic alphaviruses VEEV has a history of causing large outbreaks in large portions of South and Central America, causing thousands of human cases, with hundreds of those having neurological involvement ${ }^{15}$. Second, VEEV is also highly transmissible via the aerosol route, making it a concern for both accidental exposure as well as purposeful misuse ${ }^{21}$. This high level of transmissibility made VEEV a target for bioweapon by both the United States and former Soviet Union during the Cold War, leading to its classification as a select agent, a classification it shares with EEEV ${ }^{22}$. VEEV generally causes a mild febrile illness, that occasionally results in encephalitic infection, with encephalitic infection resulting in death in approximately $10 \%$ of cases $^{7}$. Cases of VEEV with neurologic 
involvement are often fatal, and those patients that do survive are likely to suffer from long term sequelae ${ }^{23}$.

There are currently no treatments or preventive measures available for any alphavirus, making them targets of antiviral drug and vaccine development. The pathology of alphaviruses is outlined in Figure 1. 
Old-World

long symptom duration

rarely fatal
Both

febrile illness
New-World long term sequellae sometimes fatal

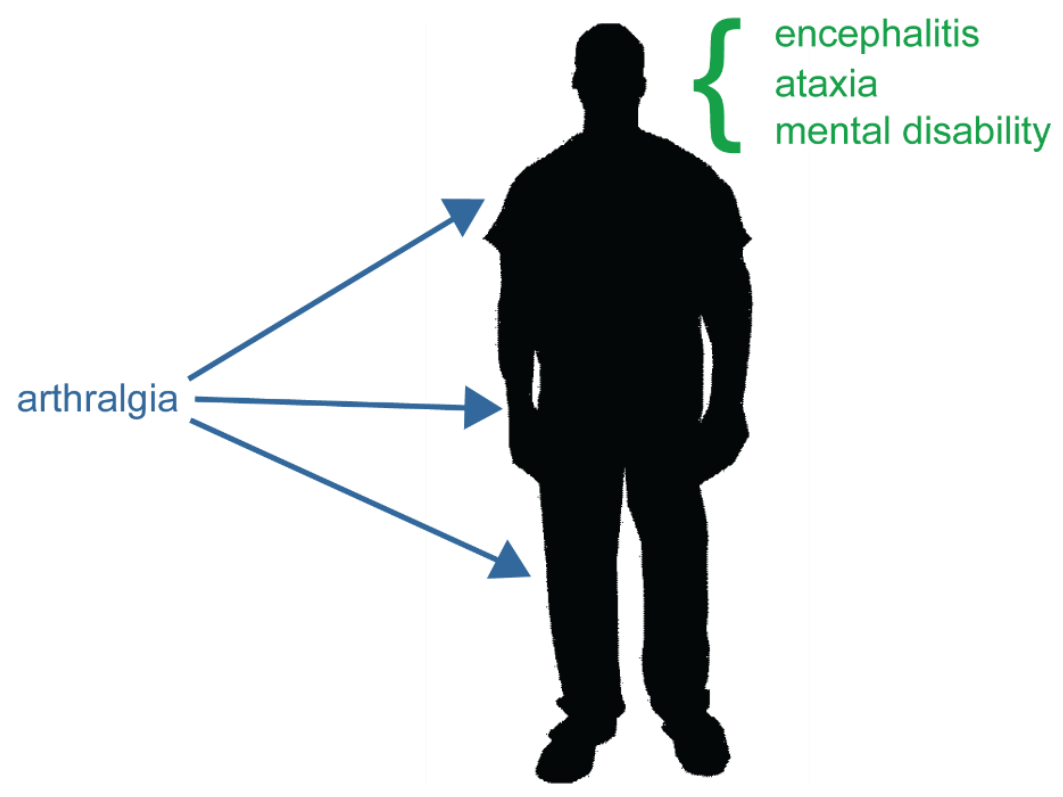

Figure 1. Alphaviral disease. Alphaviruses of both the Old and New-World clades are known to cause febrile illness in most cases, and many of these cases do not progress further. In severe cases, the Old-World viruses can cause long lasting symptoms, primarily a multijoint arthralgia that can last for several months. However, the Old-World viruses are rarely fatal. Severe cases of the New-World viruses can cause multiple neurological symptoms. In the case of neuroinvasion the New-World viruses are frequently fatal, and survivors generally have permanent sequalae. 


\section{Natural Transmission of the Alphaviruses}

Alphaviruses are vector borne viruses that generally require the use of an intermediate species to transmit to a naïve vertebrate host $^{7}$, and this transmission cycle is outlined in Figure 2. Due to this cycle, the viruses must efficiently infect and replicate in multiple species. Alphaviruses infect a wide variety of both vector and hosts, and the species infected are specific for each virus. The reservoir species for VEEV are primarily various rodents including cotton rats ${ }^{24,25}$ Individual alphaviruses are often able to infect multiple different vector species, with separate species being involved in endemic maintenance and others being necessary to cause epidemic and epizootic transmission ${ }^{26}$. Epidemic strains of VEEV have been isolated from mosquito species of several genera including Aedes, Ochlerotatus, and Psorophora ${ }^{24,25}$. These vectors where epidemic strains are found are commonly referred to bridge vectors, as these species have increase promiscuity in their feeding habits, making them more likely to infect a non-reservoir species, such as a human or an equid ${ }^{27-29}$. The feeding habits of vector species, as well as the natural range of vector and reservoir species are the primary determinants of the geographic range of any given alphavirus.

The virus will first enter the mosquito or other vector through a blood meal that is taken from an infected host. The virus will then encounter the cells of the mosquito midgut, before passing into the hoemocel, the circulatory system of the mosquito. Eventually virus will arrive in the salivary glands where it replicates to high levels and is transmitted to the next vertebrate host during a blood meal ${ }^{30,31}$. Not only does vector transmission complicate control of these viruses, but infection of the mosquito is an important selection process, and different strains of these viruses can behave differently in the vector. In particular, epidemic VEEV strains behave very differently in the mosquito than those are isolated from enzootic infection ${ }^{30-32}$. 
Upon blood meal from an infected mosquito, the alphavirus is injected into the skin of a naïve host. As these viruses have various cellular tropisms that will result in differing pathologies, here the general series of events that will occur for the infection of a susceptible and permissive host cell will be described. 


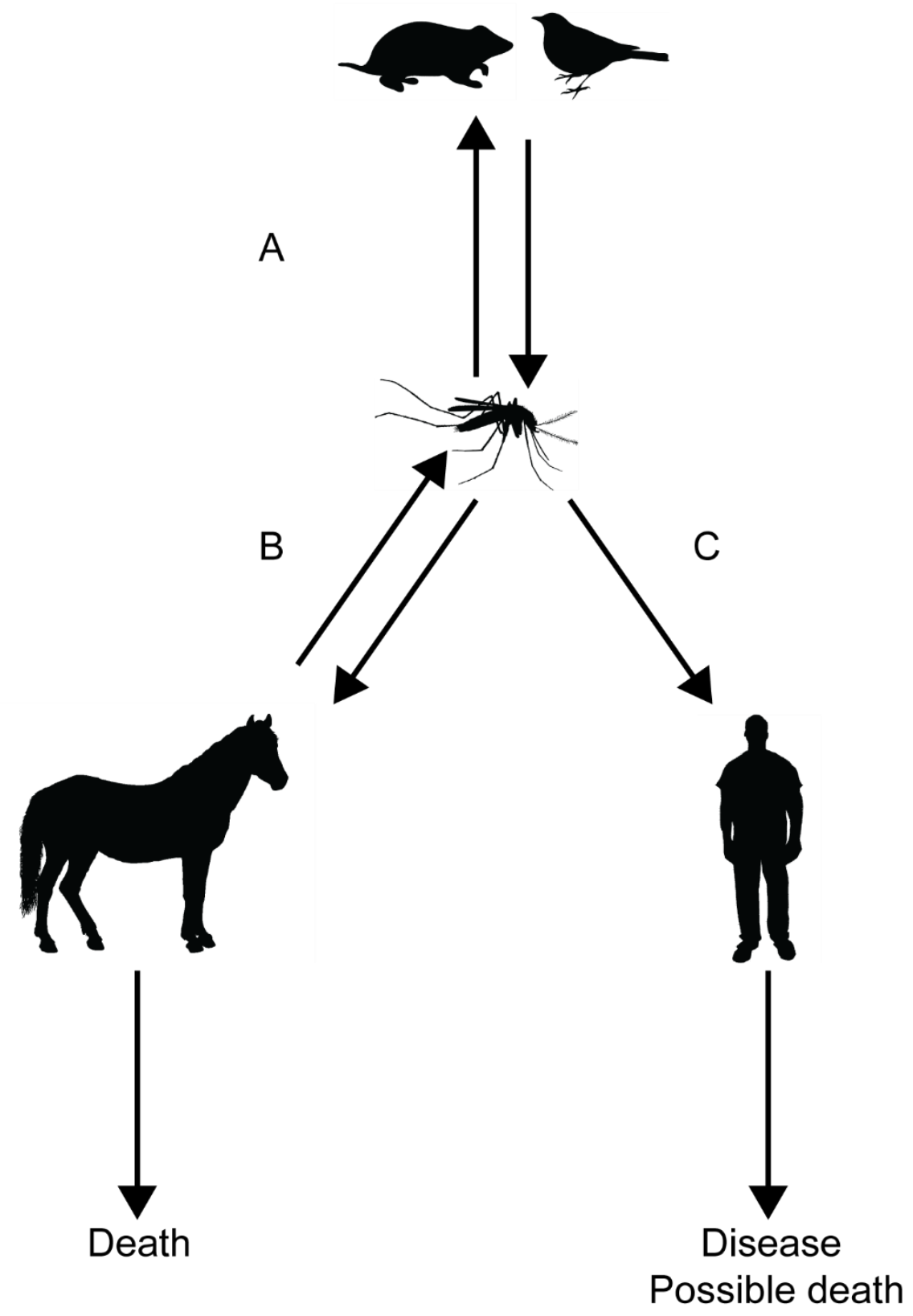

Figure 2. The transmission cycle of alphaviruses. A) Alphaviruses are maintained in nature by cycling between a host species, typically a bird or small mammal, and a mosquito vector species. B) Spillover events often occur into livestock, which reach high viral titers and readily transmit the virus to additional vectors. In the case of the New World viruses this infection almost always leads to death. C) Typically, after infection of 
livestock, humans that work in close association with these animals can also be infected by vector species. Humans are regarded as dead end hosts for VEEV. In humans these infections may lead to disease, and, in severe cases, death. 


\section{Alphaviral replication}

After inoculation into the vertebrate host alphaviruses enter permissive and susceptible host cells to manufacture new virions. This process is here described in detail, and a summary can be found in Figure 4.

\section{Receptor-mediated endocytosis}

The primary mechanism by which alphaviruses enter naïve host cells is via receptor mediated endocytosis ${ }^{33}$. There have been multiple different suggested receptors for cellular entry ${ }^{33}$. Two important receptors are DC-SIGN and L-SIGN, which are likely involved in the myeloid cell tropism displayed by VEEV, which is known to infect dendritic cells early in infection ${ }^{34}$. Multiple other receptors have been found, and continue to be found in recent work ${ }^{35}$.CRISPR has been used to great effect in the search for additional alphaviral receptors, with Mxra8 being found to be important for multiple alphaviurses ${ }^{36}$, and LDLRAD3 having been described as a receptor for VEEV specifically ${ }^{37}$. Heparin sulfate has also been found to be a binding partner for some of these viruses, and is ubiquitously expressed ${ }^{33}$. However, it has been found that viruses that have not been adapted to cell culture have less affinity for heparin sulfate $38,39,40$. This indicates that it is likely that the amount of heparin sulfate binding that has been reported is an artifact of cell culture adaptation of virus that has been produced and expanded in in vitro systems.

After receptor binding the alphaviruses are then transported into the cell via clathrin mediated endocytosis ${ }^{4133}$, resulting in a virus containing endosome passing through the stages of acidification and maturation. The New-World viruses, including VEEV, remain in the vacuoles until they reach the endosome stage, whereas the OldWorld viruses escape from the early endosomal compartment ${ }^{42}$. 
There is some evidence that alphaviruses may demonstrate alternative entry strategies, such as direct entry at the host cell plasma membrane ${ }^{4344}$. This has been well characterized in CHIKV as well, with research indicating that even though an acidification step is required, it can occur in a manner that is independent of the activity of clathrin ${ }^{45}$. However, the importance of this entry method remains unclear.

\section{Fusion/ Uncoating and RNA release}

Fusion of the viral and host cell membranes is achieved by the activity of the E1 protein, and expression of E1 without the other glycoproteins is enough to mediate viral membrane fusion ${ }^{46,47}$. This fusogenic activity is initially prevented by the interaction of E1 with $\mathrm{E} 2$, but this interaction is disrupted at low $\mathrm{pH}^{33,48}$.

After fusion the nucleocapsid core is released into the cytoplasm of the cell. The disassembly of the nucleocapsid is enhanced by the low $\mathrm{pH}$ environment, which may be

caused by membrane pores induced by $E 1^{4950,5152}$. After escape from the endosome, the nucleocapsid interacts with ribosomes, which disassemble the capsid in a non-catalytic manner ${ }^{53}$. This disassembly appears to be dependent on conserved sequences in the capsid protein ${ }^{54}$. These steps have only been outlined so far using SINV and SFV.

\section{Translation and processing of the nsPs}

As positive sense, single-stranded RNA viruses, the alphavirus genome requires no additional processing to be available as an mRNA for translation by the host cell ribosomes. The genome has both a 5' methyl cap and a 3' poly A tail, allowing for normal recruitment of initiation and elongation factors to begin translation ${ }^{7}$. The initial step of viral replication is the translation of the nonstructural polyprotein, which contains the proteins which are responsible for the replication of the viral RNA. The viral nsPs are numbered in the order that they occur in the genome from $5^{\prime}$ to $3^{\prime}, 1-4$. The genomic 
organization of VEEV can be found in Figure 4. The initial polyprotein is translated as either nsP123 or nsP1234, depending on read through of a stop codon that may or may not be present in the genome depending on the alphavirus in question ${ }^{55,56,57}$.

After the initial translation of these proteins, they undergo tightly controlled cleavage events that result in the formation of multiple intermediates as well as the formation of the final mature replicase complex nsP1/2/3/458,59. This fully cleaved, mature complex is highly stable. Control of this cleavage process is important as it controls the levels of viral RNA species that are present at different times during infection ${ }^{60,61}$. This cleavage process appears to have unique regulatory features such as having morphological cleavage recognition instead of sequence specificity ${ }^{62}$. This regulatory process is highly important to viral biology as altering it leads to attenuation ${ }^{63}$. Proper cleavage is also important to immune evasion, as viruses with incomplete cleavage result in alterations of the viral RNA species prevalences, increasing Type I interferon induction as well as sensitivity of the viruses to interferon ${ }^{64}$. 


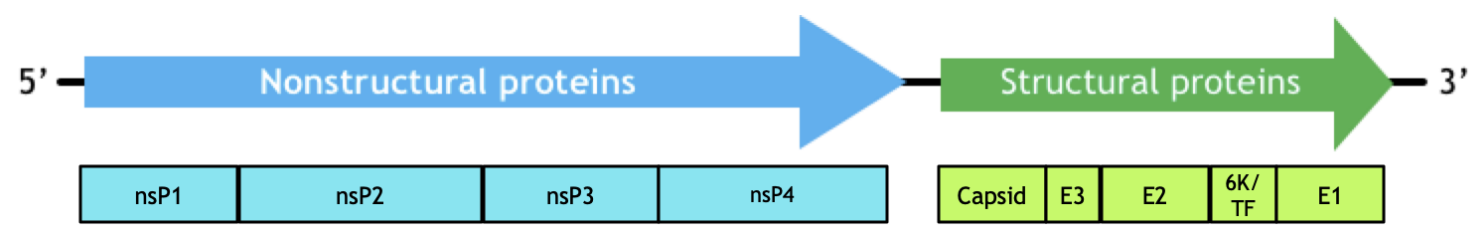

Figure 3. The genetic structure of VEEV. VEEV has a 12kb, linear, positive-sense genome. The genome has two open reading frames, the nonstructural and the structural. The nonstructural open reading frame encodes the four nonstructural proteins, which are responsible for replication of the viral RNA. The structural open reading frame encodes for the $\mathrm{E}$ proteins and capsid as well as the $6 \mathrm{~K}$ and TF proteins. The capsid and $\mathrm{E}$ proteins form the structure of the viral particle. 


\section{Viral RNA Replication}

The process of viral RNA synthesis is outlined in Figure 5. To make additional molecules of RNA genome, the virus is required to first transcribe the positive-sense genome into negative-sense template strand. This activity is performed by the partially cleaved polyprotein nsp123/4 ${ }^{61}$. However, nsP2 has protein cleavage activity which rapidly degrades the polyprotein into its constitutive parts. This initially produces intermediate forms of the replicase complex that are short lived and produce both negative and positive-sense RNA ${ }^{60,61}$. The final cleavage between nsP2 and nsP3 leads to the formation of the mature replicase complex nsP1/2/3/4 which produces only positive-sense RNA ${ }^{58,59,65}$. This self-proteolytic behavior creates a distinct expression profile of the viral RNA. Initially the immature forms of the complex produce higher levels of negative-sense RNA. As the complex is processed the synthesis of negative-sense RNA is reduced and eventually eliminated. This causes most negative-sense RNA to be produced early in infection, as well as less negative-sense RNA being produced overall $^{66}$.Following cleavage and assembly of the mature replicase complex, RNA synthesis converts to the synthesis of positive-sense genomic and subgenomic RNA ${ }^{67}$.

The positive-sense genomic RNA functions primarily as the genetic material of the next generation of virus, as well as being translationally activity in the cell to produce additional nonstructural proteins. However, recent work has indicated that the genomic RNA may have biological functions in addition to this, as increasing the amount of noncapped genomic RNA that is produced during infection leads to a decrease in viral fitness ${ }^{68}$. The other positive-sense viral RNA that is produced is the subgenomic RNA. This small RNA is produced from a separate promoter and encodes for the structural genes of the virus ${ }^{55}$. 


\section{Localization of genome replication}

Alphaviruses demonstrate a sequestration of their replication to intracellular membranes, which is similar to other RNA viruses which also largely replicate in and on membranous structures ${ }^{69-71}$. The alphaviruses utilize microinvaginations called spherules ${ }^{69}$. These are sites where the viral RNA has been found to localize in infected cells ${ }^{72,73}$. It has been confirmed in vitro that these structures contain viral RNA synthetic activity through the use of purified spherules to synthesize viral RNA ${ }^{74}$. It has been recently determined that the initial formation of the spherules is dependent solely on the activity of the nsPs with no requirement for viral RNA being present ${ }^{75}$. However, the size of the individual spherules is dependent on the length of the RNA that is transcribed within, which appears to be a feature unique to alphaviruses ${ }^{76}$.

Spherules were initially identified on large, endosomal-like compartments in infected cells. In several of these viruses these spherules form at the plasma membrane and later traffic to intracellular compartments ${ }^{77}$. This localization has not yet been fully characterized in VEEV. In vertebrate cells, the movement of the spherules away from the plasma membrane is dependent on the activation of PI3K-Akt-mTOR, and reduction of this activation is associated with an increased proportion of the spherules remaining at the cellular membrane ${ }^{78}$

\section{Translation of the structural genes}

The structural genes of the alphaviruses are produced via translation of the subgenomic RNA. The initial gene product is a polyprotein that contains the capsid, E proteins, $6 \mathrm{~K}$, and TF proteins protein ${ }^{557}$. The capsid protein contains a serine protease domain and uses this to rapidly cleave itself from the other structural genes ${ }^{55}$. The capsid protein forms the nucleocapsid core and is responsible for binding to and 
packaging viral $R N A^{61}$, this interaction is mediated by the size and charge of the RNA molecule ${ }^{79}$. In addition to the role that it plays in virion structure, the capsid of alphaviruses also has biological functions involved in viral pathogenesis. In the NewWorld alphaviruses the capsid protein is able to block the nuclear pores and thus prevent the translation of new cellular protein, enhancing viral pathogenesis, cytopathic effect, and assisting in immune evasio ${ }^{80,81}$. This protein synthesis inhibition functions in tandem with the nsPs which actively inhibit the synthesis of cellular proteins ${ }^{82}$.

After cleavage of the capsid protein, the glycoproteins are translated into the endoplasmic reticulum, and pass through the Golgi apparatus before being embedded into the plasma membrane of the cell ${ }^{55}$. These proteins are also highly posttranslationally modified via glycosylation and palmitoyaltion ${ }^{55}$.

\section{Packaging of the viral RNA and release of the virion}

After translation of the structural proteins, the viral RNA must be packaged into the virion and then released to infect new host cells. The RNA and capsid undergo interactions due primarily to molecule size and charges, resulting in nucleocapsid like structures occurring in the cytoplasm ${ }^{55,79,83}$. Alphaviruses bud directly from the plasma membrane of the infected cell 7 . However, it is unclear how this budding process is initiated $^{83}$. It has been found that both the preformed nucleocapsid like structures and the glycoproteins are able to drive budding ${ }^{83,84}$. However, when either of the functions occurs independently of the other, there is a marked reduction in efficiency, indicating that it is likely that these two mechanisms interact to allow for the maximal budding of virions ${ }^{84}$

Transport of the structural proteins to the plasma membrane requires the host secretory system ${ }^{84}$. However the exact proteins that are used remain unknown ${ }^{84}$. 
Release of virions can also be inhibited by host proteins. In particular tetherin has been shown to prevent the release of virions from infected cells ${ }^{84}$. The general replication of alphaviruses is outlined in Figure 4 below. 


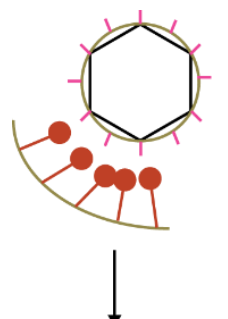

Receptor

Mediated

Endocytosis

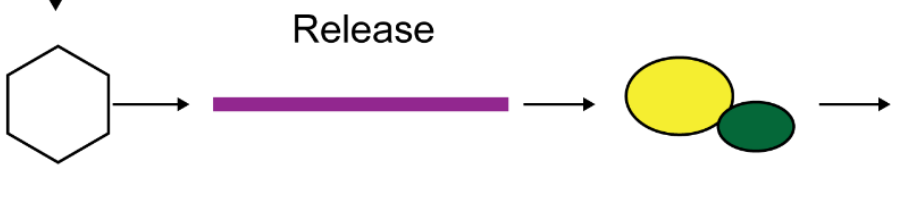

RNA

nsP Translation RNA Replication in Spherules

$\bigcirc \rightarrow$

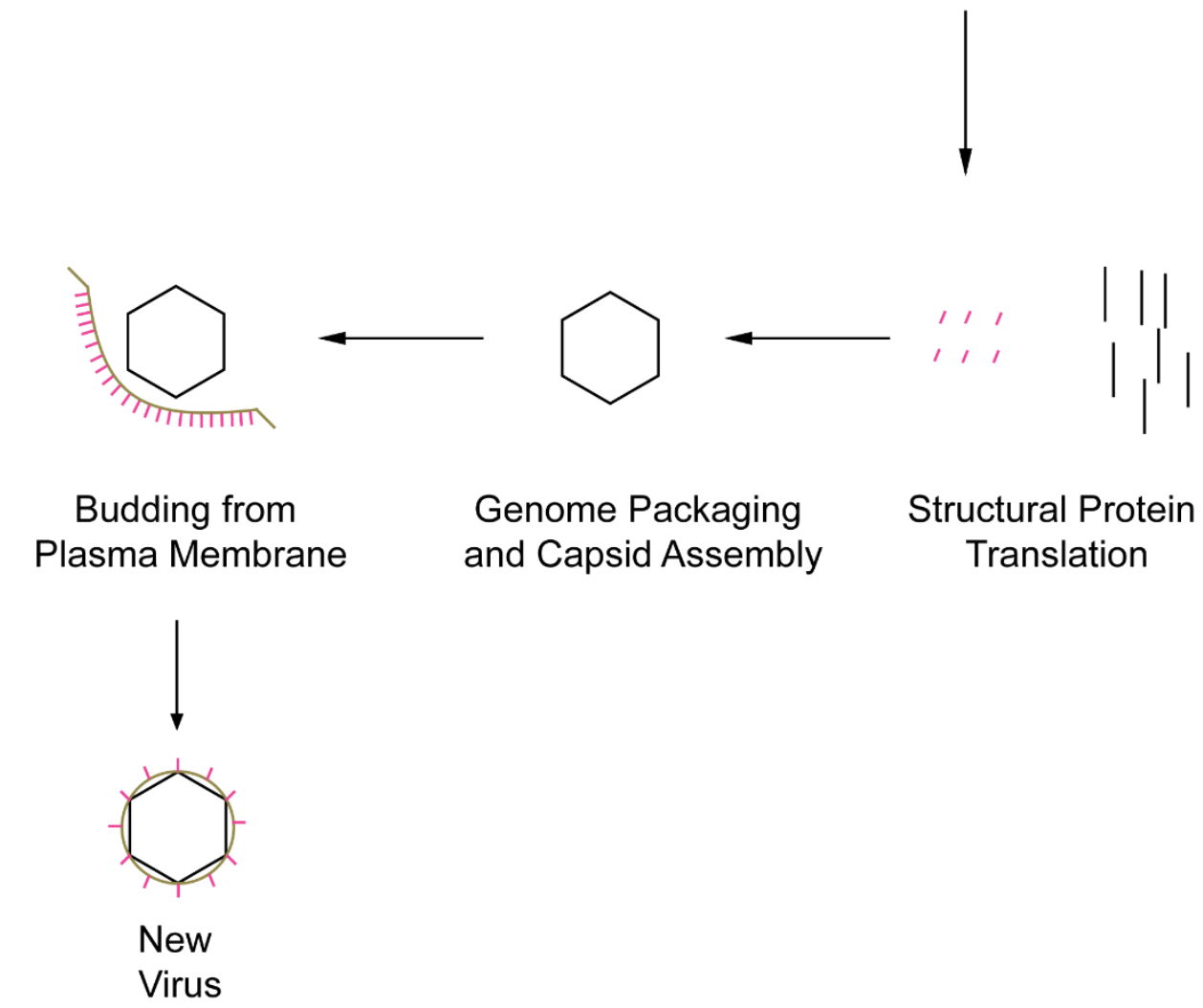

Figure 4. The replication cycle of alphaviruses. The virion enters a susceptible cell via receptor mediated endocytosis, and due to $\mathrm{pH}$ changes of the endosome releases its RNA into the cytoplasm of the host cell. The positive sense genomic RNA is first used by ribosomes to translate the viral nsPs as a polyprotein. The polyprotein will undergo cleavage events that control the synthesis of the viral RNA species. This RNA synthesis 
occurs in membrane invaginations that are termed spherules. These spherules protect the viral RNA and nsPs from detection by the host cell. Late in infection the structural genes are synthesized. The capsid will form into nucleocapsid cores as it packages the viral RNA, and the glycoproteins are transported to the cell membrane. The nucleocapsid cores translocate to the cellular membrane where they bud off, collecting their envelope and glycoproteins and forming new infectious virus. 


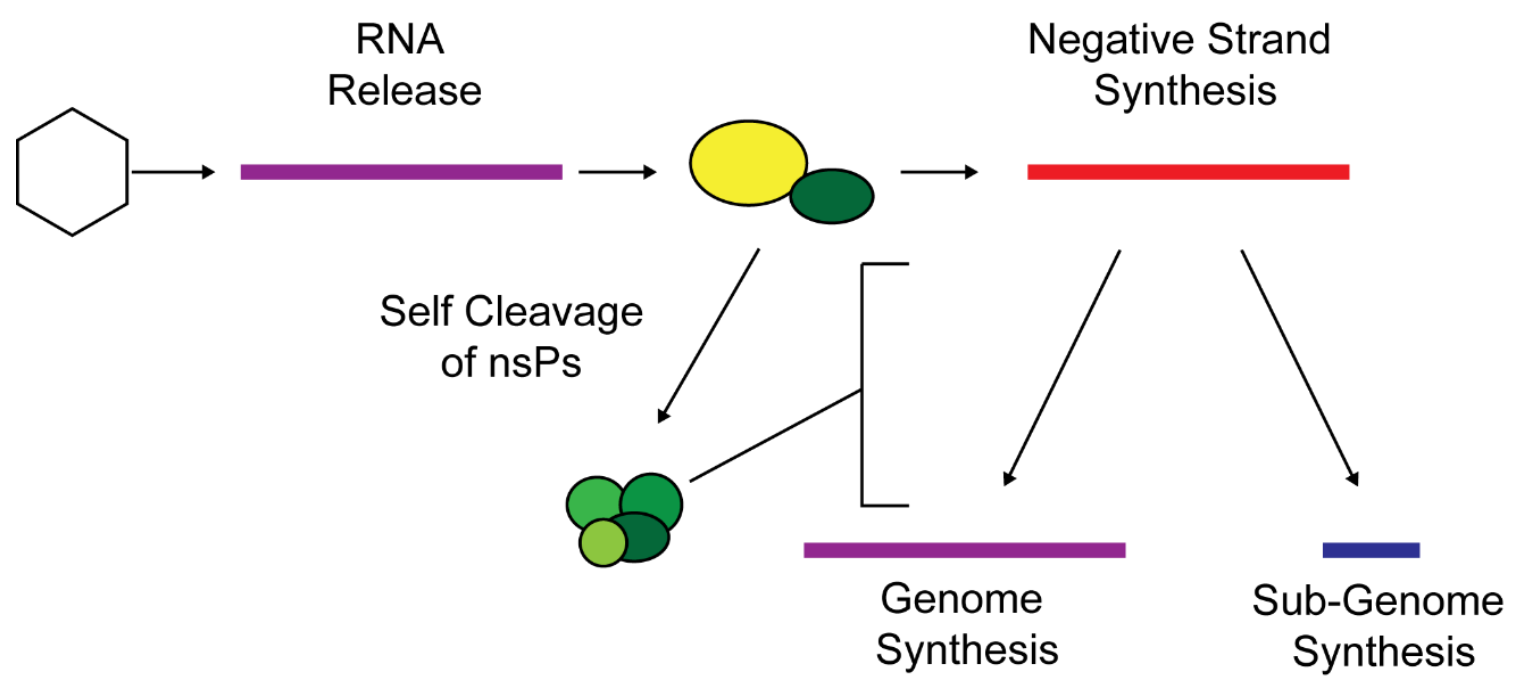

Figure 5. RNA synthesis of alphaviruses. This RNA synthesis activity is carried out in spherules on the membranes of cellular organelles. After release into the cytoplasm the genomic RNA is used to synthesize the initial nonstructural polyprotein. nsP2 initially cleaves between nsP3 and 4 leading to nsP123/4, which synthesizes primarily negativesense template RNA. The protein undergoes rapid cleavage through intermediate states to reach the final replicase complex nsP1/2/3/4. This complex synthesizes new positivesense genomic and subgenomic RNA and can no longer synthesize negative-sense RNA. The genomic RNA is used to synthesize additional genomes and is packaged into progeny virions. The subgenomic RNA is used to synthesize the structural genes that from the new virions. 


\section{Functions of the alphaviral nonstructural proteins}

The alphaviruses make four nonstructural proteins. These proteins are responsible for viral RNA replication as well as many other enzymatic functions. The nonstructural proteins are also intimately involved in the pathogenesis of the alphaviruses. The functions of these proteins will now be described in greater detail. While the functions of the nsPs are highly conserved, differences between the Old and New-World viruses will be indicated when necessary.

\section{Nonstructural protein 1}

NsP1 is the capping enzyme for the viral genomic RNA, and this activity occurs independent of the activities of the other $\mathrm{nsPs}^{85}$. The activity of this protein has only recently been examined in VEEV, having previously been studied only in Old-World viruses. This was also the first time that each individual step, including the final guanyl transfer, has ben detected ${ }^{86}$. The steps occur as follows. 1) The transfer of a methyl group from S-adenosylmethionine to position N7 of a molecule of GTP is catalyzed 2) nsP1 receives the methyl-GTP becoming guanylated, releasing pyrophosphate in the

process 3) the 7 methyl-GMP is transferred to the $5^{\prime}$ end of the target RNA ${ }^{86,87}$. For this reaction to occur properly, the RNA being capped must have had its 5' terminal phosphate removed by nsP2 ${ }^{88} \mathrm{NsP} 1$ is also responsible for the anchoring of the viral replicase complex to cellular membranes which are the site of RNA replication, and this activity is required for capping to be carried out as well ${ }^{89-91}$. Very recently a cryo-em structure was published that showed how nsP1 influences the structure of the membrane spherules and potentially controls entry and exit of materials ${ }^{92}$. NsP1 was found to form a ring-like structure that appears to act as a gate and controls entry and exit from the spherule ${ }^{92}$. The nsPs are also responsible for the formation of the 
spherules and this can occur in the absence of viral $\mathrm{RNA}^{75}$ however the viral RNA determines the spherule $\operatorname{size}^{76}$.

\section{Nonstructural protein 2}

NsP2 is a multifunctional protein with many described functions and multiple domains with discreet enzymatic activities. First, nsP2 is responsible for host cell transcriptional shutoff in the Old-World viruses, and loss of this phenotype reduces viral cytotoxicity ${ }^{80,93}$. In the New-World viruses this activity is instead carried out by the capsid protein, and nsP2 is responsible for shutoff of host cell protein synthesis, and may have a role in packaging of viral $\mathrm{RNA}^{80,81,94,95}$. In VEEV this translational shut down provides resistance to a pre-existing antiviral state ${ }^{94}$.

There are three recognized domains in nsP2. The $\mathrm{N}$-terminal regions contains a helicase domain, and NTPase activity that serves to provide energy for the helicase ${ }^{96,97}$. This same region also has RNA 5'-triphosphatase activity which prepares RNA for capping, allowing for translation and packaging in virions ${ }^{88}$. The $\mathrm{N}$ terminal region of the protein including the helicase domain has recently been crystalized ${ }^{98}$. The most interesting feature was the large number of accessory domains that were present, as these domains had not previously been predicted by structural modeling. In particular the so called stalk domain, which based upon our own research appears to have an important function in viral RNA synthesis ${ }^{66}$.

Large portions of the $\mathrm{N}$-terminal region of nsP2 remain poorly characterized. Studies have implicated that in VEEV this region may be important to packaging of the viral genome ${ }^{95}$. However, in SINV this a transposon insertion approach using the sequence for GFP found that region was involved in the cleavage between nsP2 and nsP3, controlling the ratio of genomic and subgenomic RNA, and regulation of RNA 
synthesis $^{99}$. This range of phenotypes indicates that these regions are highly important to these viruses, but further characterization and research is needed.

NsP2 also contains a cysteine protease domain that is responsible for the cleavage of the nsPs from the polyprotein into its constitutive members ${ }^{100-102}$. As described earlier, this cleavage is responsible for the transition from the synthesis of negative-sense viral RNA to positive-sense viral RNA ${ }^{61,103}$. The protease has also been shown to target cellular proteins, a common feature of viral proteases, and this is related to resistance to innate immune responses ${ }^{104}$.

Lastly, nsP2 contains a putative methyltransferase domain that was predicted due to the structure of the protein ${ }^{102}$. However, it is predicted to be inactive as it lacks the active site residue necessary for activity ${ }^{85}$. Recently though, there has been work that indicates this domain may play a role in interferon shutoff due to its interaction with signal transducer and activator of transcription proteins 1 (STAT1) ${ }^{105}$. This activity appears to be mediated by enhancing the nuclear export of STAT1, which prevents the magnification of downstream immune signaling, including the interferon response ${ }^{105}$

\section{Nonstructural protein 3}

NsP3 is poorly understood, but mutations within this protein have resulted in defects in both negative-sense and subgenomic RNA synthesis ${ }^{106}$.

NsP3 contains a macrodomain with both adenosine diphosphate ribose (ADPr) binding and hydrolase activity ${ }^{85,107108}$. This ADP ribosylase activity is necessary for infection of neural cells and the hydrolase activity results in an increase in replicase complexes $^{109}$. In a SINV model, reductions in hydrolase activity led to reduced neurovirulence while increases in ADP ribosylase activity increased neurovirulence ${ }^{110}$. 
NsP3 also contains the highly conserved alphavirus unique domain, or AUD ${ }^{85}$. This domain is maintained across all alphaviruses ${ }^{85}$. Recent work has indicated that it potentially has many functions in CHIKV, particularly in subgenomic RNA replication ${ }^{111}$. Disruption of the AUD resulted in decreased infectivity, potentially due to decreased interaction with the viral RNA and the subgenomic promoter ${ }^{111}$.

The last feature of note in nsP3 is the hypervariable domain or HVD. This domain is so varied that it can be distinct between strains of a single viral species, such as in VEEV ${ }^{112}$. This region is tolerant of significant mutation and even deletion, which is unique compared to the rest of the alphaviral genome ${ }^{113}$. Natural duplications and insertions in this region can even have positive effects on viral fitness ${ }^{114}$. The HVD is also involved in interaction with host cell proteins, resulting in the formation of distinct protein complexes in Old and New-World viruses ${ }^{115}$. These interactions include the cellular machinery responsible for the formation of stress granules, which alphaviruses utilize to their own replicative benefit ${ }^{116-119}$. These interactions are highly specific to viral species as well, and may partially drive the differences in pathogenesis seen between species $^{119-121}$.

\section{Nonstructural protein 4}

NsP4 is produced in small amounts by most alphaviruses due to the inclusion of a stop codon between nsP3 and nsP4 ${ }^{55,57}$. Altering expression levels of nsP4, either to increase or decrease expression, decreases viral fitness, indicating that tight control of expression is highly important ${ }^{122}$. The tight limit on expression of nsP4 is also promoted by it being targeted by $\mathrm{N}$-end rule degradation, further limiting the amount present in the cell $^{123}$. 
NsP4 is the RNA dependent RNA polymerase (RDRP) of the alphaviruses and is active in both positive and negative-sense RNA synthesis, with the specificity being determined by the cleavage state of the other $\mathrm{nsPs}^{61,85,124}$. NsP4 can display RNA synthesis activity alone, but the activity is enhanced by the presence of the other $n \mathrm{Ps}^{124-126}$.

NsP4 also has a large $\mathrm{N}$ terminal region that lacks predicted structure or function. Our own work has predicted that this region is somehow involved in viral RNA synthesis, as mutations in this region result in resistance to the effects of a drug that inhibits the production of new viral RNA ${ }^{66,127}$. However, the function of this region remains unclear. Work by others has also shown that mutations in this regions have a broad range of effects on viral RNA synthesis ${ }^{128}$. This work, as well as the antiviral resistant VEEV isolates that have been recovered in our lab ${ }^{127}$ indicate that this region plays an important role in RNA synthesis of these viruses potentially in tandem with nsP2. This indicates that these proteins have additional, complex interactions and roles in viral biology that remain to be understood. The functions of the nsPs are summarized in Table 1. 


\begin{tabular}{|l|l|l|}
\hline Protein & Structures & Functions \\
\hline nsP1 & $\begin{array}{l}\text { methyl transferase } \\
\text { domain, guanyl } \\
\text { transferase domain, } \\
\text { membrane association } \\
\text { domains }\end{array}$ & $\begin{array}{l}\text { caps viral RNA making it } \\
\text { usable by ribosomes, } \\
\text { anchors the replication } \\
\text { machinery to cellular } \\
\text { membranes }\end{array}$ \\
\hline nsP2,85-87,89-92
\end{tabular}

Table 1. Summary of alphavirus nonstructural protein functions 


\section{Current state of alphaviral therapy development}

Currently there are no approved therapeutics or vaccines for alphaviral disease. The development of treatments is urgently needed due the global range of alphaviruses and their continuous emergence into naive populations. This is compounded with the high risk of alphaviruses expanding their geographic ranges due to climate change. There have been many investigations into potential alphaviral therapeutics; however thus far, none have resulted in clinically approved treatments.

Several studies have examined the efficacy of currently approved broad spectrum antiviral treatments. Ribavirin, a common standard of care in the treatment of viral infections, has been found to be ineffective ${ }^{131}$. Interferon $\alpha$, another common antiviral treatment, has been found to be effective in mouse models at high doses, when it is pegylated to increase its halflife ${ }^{132}$. However, interferon treatment has severe side effects, and is particularly noted for the neurological symptoms it induces, such as depression ${ }^{133}$.

A recently licensed drug of interest is the broadly anti-influenza treatment favipiravir. Created and initially tested in Japan, this is a nucleotide analogue which has been found to inhibit the polymerase of influenza viruses ${ }^{134}$. Treatment with favipiravir is mildly efficacious against WEEV, increasing survival in infected mice ${ }^{134,135}$. Also, treatment with this drug during the acute phase of infection resulted in clearance of CHIKV from infected mice, but there was no effect on the infection if treatment was initiated in the chronic phase ${ }^{136}$. It is important to note, that this treatment was not very efficient, requiring high doses of drug and delivery mechanisms that would be impractical during a natural outbreak of these viurses ${ }^{135,137}$ 
There have also been many other investigations into other potential treatments, such as therapeutic antibodies, mifiprestone analogues, and antimicrobial peptides ${ }^{138-}$ 140. Promising data has come from treatment with antagonists of argonaut 2, an important protein in RNA silencing ${ }^{141}$ and inosine-5'-monophosphate (IMP) dehydrogenase, an enzyme involved in guanine synthesis ${ }^{142131143}$. Finally, there has even been work that has examined the effects of compounds like $\beta$-d-N4Hydroxycytidine, which increases the mutation rate during viral RNA synthesis in an attempt to induce damage in the genome ${ }^{144}$. However, as mentioned above, none of these leads have led to therapies licensed for the treatment of alphaviral infection in the clinic. Of particular interest in ongoing antiviral research is the development of viral targets that can be perturbed by treatment, leading to the development of more specific antiviral therapies with minimal side effects.

\section{The development of alphaviral therapies in the Chung lab}

The Chung lab has a history of antiviral drug development, and is known for the development of high throughput screening processes for the identification and characterization of novel therapeutic compounds ${ }^{127,145-147}$. Briefly, libraries of compounds are screened for increases in the viability of infected cells compared to a vehicle control. This is performed in a 384 well format allowing for rapid screening of thousands of compounds $^{127}$. Compounds that meet the threshold for effectiveness are then assayed for antiviral effects as well as cytotoxicity, then promising compounds are further examined for pharmacological characteristics. This results in the identification of hit compounds which may become lead compounds themselves, or may be used to generate additional compounds using medicinal chemistry ${ }^{127}$.

The antiviral drug discovery work using VEEV resulted in the discovery of an initial quinazolinone hit compound that was found to be highly effective against VEEV 
with some efficacy against EEEV and WEEV as well ${ }^{127}$. Additional compounds that have unrelated mechanisms of action were also discovered using this same process, such as compound ML416, a pyrimidine analoge ${ }^{146}$. The initial quinazolinone compound was then further developed in collaboration with a medicinal chemist to the current lead compound ML336 $6^{66,148}$. The hit compound was also used in several basic assays of compounds functionality and it was found to act primarily early in infection. This, combined with sequencing data from resistant viral isolates, indicated that the hit compound, and its derivative ML336, were potentially acting to inhibit viral RNA synthesis in the infected cell ${ }^{148}$. The characterization of ML336 and its activity in the host cell will be discussed at length in the following chapter. A selection of compound structures that were used in this characterization work is included in chapter 2.

The Chung lab not only tests these compounds as potential therapeutics, but also utilizes this expertise in antiviral drug discovery to probe viral biology. The antiviral compounds identified and developed in this process can be used as probes to produce mutant viruses that resist drug treatment ${ }^{148}$. These mutant isolates can then be sequenced and used to examine previously unknown biological activities of the viruses used. This process is described in detail in chapter 4 of this work. Where an anti-VEEV compound was used to probe the nonstructural proteins for novel biological functions.

\section{Objective of Dissertation}

Alphaviruses are important pathogens of both human and animal hosts and are of particular concern due their potential use in biological warfare. Several of these viruses are also rapidly expanding into naïve populations. There are currently no approved vaccines or antiviral strategies available for the treatment or prevention of 
these viruses. New potential therapeutics need to be characterized and further developed. Our group has developed and begin to examine several anti-VEEV compounds and these require more complete study to determine their mechanism(s) of action ${ }^{127}$. Novel antiviral compounds also have the potential to be used as probes to characterize alphaviral biology. This is primarily carried out by the isolation and characterization of compound resistant viruses. Mutations in these viruses can be identified ${ }^{127}$ and any alterations in the behavior of these viruses can be mapped to the mutated region(s). These avenues of research were explored in the context of the following aims:

1) To determine the mechanism of action of the anti-VEEV benzamidine compound ML336.

2) To characterize the hypothetical interaction between amidine scaffold compounds and the nonstructural proteins of VEEV.

3) To describe the biological function of the regions of nsP2 which were found to be important to ML336 activity.

\section{Justification}

Alphaviruses are important pathogens of both humans and domesticated animals, and the lack of therapeutic and preventative options means that there is a significant need for research to find and characterize new potential therapeutics and drug targets.

The design of antiviral drugs has been historically rather difficult, as the pathogens utilize predominantly host systems for their biological processes. While there 
has been some success with antiviral development recently, these drugs often have significant side effects making them poorly tolerated and underutilized ${ }^{149,150151152153 .}$

The current lack of anti-VEEV treatments, as well as the generally poor track record of many antiviral compounds, have led to our own interest in developing better antiviral compounds, with a focus on alphaviruses generally and VEEV specifically. This virus is not only a risk of natural infection, but is also a high-risk agent for misuse, making the development of treatments high importance.

Further, there are many areas of alphaviral biology that remain poorly defined, despite extensive study. Antiviral compounds can be used to derive treatment resistant viruses. Those viruses that resist treatment can be sequenced for mutations in their genomes, and then these mutants characterized by classical virological methods. This method has the advantage of only discovering mutations that are compatible with replication, avoiding the creation of nonviable mutants which is common in random mutagenesis studies. With the characterization of these isolates, new information about the function of the viral proteins and their interaction with each other and cellular components can be gained. 


\section{CHAPTER 2}

BENZAMIDINE ML336 INHIBITS VEEV RNA SYNTHESIS

Skidmore AM, Adcock RS, Jonsson CB, Golden JE, Chung DH. Benzamidine ML336 inhibits plus and minus strand RNA synthesis of Venezuelan equine encephalitis virus without affecting host RNA production. Antiviral Res. 2020 Feb;174:104674. doi: 10.1016/j.antiviral.2019.104674. Epub 2019 Dec 6. PMID: 31816348; PMCID: PMC6935354. 


\section{Overview}

Venezuelan equine encephalitis virus (VEEV) is an alphavirus that is endemic to the Americas. VEEV outbreaks occur periodically and cause encephalitis in both humans and equids. There are currently no therapeutics or vaccines for treatment of VEEV in humans. Our group has previously reported on the development of a benzamidine VEEV inhibitor, ML336, which shows potent antiviral activity in both in vitro and in vivo models of infection. In cell culture experiments, ML336 inhibits viral RNA synthesis when added 2-4 hours post-infection, and mutations conferring resistance to this activity occur within the viral nonstructural proteins (nsP2 and nsP4) ${ }^{127}$. This led us to hypothesize that ML336 targets the viral replicase complex and inhibits viral RNA synthesis. Using ML336 and structurally related compounds, we demonstrate that the cellular antiviral activity of this antiviral scaffold correlates with inhibition of viral RNA synthesis. However, ML336 has no effect on the RNA synthesis of the closely related CHIKV or on cellular RNA synthesis. With a combination of fluorography, strand-specific qRT-PCR, and tritium incorporation, we demonstrated that ML336 inhibits the synthesis of the positive-sense genomic, negative-sense template, and subgenomic RNAs of VEEV. In summary, ML336 and related compounds inhibit all stages of VEEV RNA synthesis during infection, and this activity mediates the antiviral effect of these compounds.

\section{Introduction}

Venezuelan equine encephalitis virus (VEEV) is a single-stranded, positivesense, RNA virus belonging to the family Togaviridae, which includes other medically important mosquito-borne viruses such as Chikungunya virus (CHIKV), and Eastern and 
Western equine encephalitis viruses (EEEV and WEEV respectively) ${ }^{7}$. The encephalitic alphaviruses, VEEV, WEEV, and EEEV, are closely related, sharing a recent common ancestor as determined by phylogenetic analysis ${ }^{154}$. VEEV generally causes a mild febrile disease, while approximately $1 \%$ of patients suffer from encephalitis, leading to death in about $10 \%$ of these encephalitic patients ${ }^{7}$. Only supportive care is available for those with VEEV infection.

Encephalitic alphaviruses have caused periodic outbreaks in the Americas throughout the $20^{\text {th }}$ century, and of these viruses VEEV has been the most significant public health burden. Historically, large VEEV outbreaks occur about every 15 - 20 years, typically affecting thousands of equids and humans. For example, between 1962 and 1972 in Central America ${ }^{15}$, over 109,000 human cases of VEEV were reported, with nearly 1,000 neurological cases and over 500 associated human fatalities. These outbreaks also caused a significant burden to the agricultural industry with over 800,000 reported deaths of equids due to VEEV infection.

In addition to large natural outbreaks, VEEV poses additional threats to the public. VEEV is classified as a Select Agent by both the Centers for Disease Control and Prevention and United State Department of Agriculture for its historic and potential use as a bioweapon ${ }^{22}$. There are currently no licensed treatments or vaccines for VEEV, or any other alphaviral infection in humans. Due to the stochastic nature of VEEV outbreaks, and difficulty in defining target groups for vaccination, therapeutics could be crucial for control of VEEV.

A wide variety of compounds have been reported to show anti-VEEV activities. These include mifepristone analogues, argonaute-2 antagonists, therapeutic antibodies, compounds that induce mutations in the viral genome, and naturally expressed host antimicrobial peptides to name a few ${ }^{138-140,143,155}$. Efforts using the broad spectrum, FDA- 
approved antivirals interferon and ribavirin resulted in antiviral effects only at doses that were higher than are clinically relevant ${ }^{131,132}$. Other discovery efforts using nucleoside analogues or non-nucleoside analogues have shown moderate effects against VEEV ${ }^{146,156}$. Lastly, the broadly anti-influenza drug faviprivir ${ }^{134,136}$, shows efficacy against some alphaviruses ${ }^{135}$. However, this drug has not been tested against VEEV in a mouse model, and its efficacy against these related viruses is poor, requiring doses of up to 400 $\mathrm{mg} / \mathrm{kg} / \mathrm{day}{ }^{135,157}$

To address the unmet need for VEEV therapeutics, we employed a highthroughput, cell-based, anti-VEEV screen and discovered a novel quinazolinone hit compound (CID:15997213) ${ }^{158}$. This compound showed significant promise as a small molecule inhibitor of VEEV infection with an $\mathrm{EC}_{50}$ of $1.9 \mu \mathrm{M}$ in a cell culture assay, and $100 \%$ protection of VEEV-challenged mice at a dose $50 \mathrm{mg} / \mathrm{kg}$ in a lethal VEEV infection model ${ }^{127}$. This hit compound was then further refined using medicinal chemistry approaches in pursuit of compounds with superior pharmacological characteristics. The most promising of these further derived compounds was ML336, Figure 6 A. ${ }^{159}$. ML336 shows potent and specific anti-VEEV $\left(\mathrm{EC}_{50}=32 \mathrm{nM}\right)$ activity in vitro, and VEEV titer reduction greater than 7.2 logs at $5 \mu \mathrm{M}^{145,159}$. ML336 also effectively protected mice in a lethal VEEV infection model, with no apparent toxicity at any of the examined doses ${ }^{159}$.

Our previous resistant mutation studies suggest the hit quinazolinone compound and benzamidine ML336 both inhibit VEEV replication by interfering with non-structural proteins 2 and 4 (nsP2 and nsP4) in the middle phase of replication ${ }^{127}$. NsP2 and nsP4 are essential proteins of the viral replicase complex of alphaviruses ${ }^{7,102,124}$. The incoming viral genomic RNA (49S) is translated to form the polyprotein nsP123 and nsP4. The polyprotein then synthesizes negative-sense RNA using the genomic RNA as the template strand ${ }^{61,160}$, Figure $6 \mathrm{~B}$. NsP123 rapidly undergoes cleavages mediated by the 
protease activity of nsP2 ${ }^{58,59}$, resulting in nsP1, nsP23, and nsP4, and then further into nsP1, nsP2, nsP3, and nsP4 (nsP1/2/3/4). The mature replicase complex, nsP1/2/3/4, synthesizes the positive-sense, $49 \mathrm{~S}$ viral genomic and $26 \mathrm{~S}$ subgenomic $\mathrm{RNAs}^{61,103}$. This viral RNA synthesis by the mature replicase complex occurs in spherules, microinvaginations on intracellular organelles, in the infected cells ${ }^{91,161}$. RNA-dependent RNA polymerase (RdRP) activity is carried out by nsP4, and nsP2 also exhibits nucleoside triphosphatase (NTPase) activity and helicase activity during RNA synthesis, in addition to the aforementioned protease activity for processing of the polyprotein ${ }^{88,96,162}$. 
A<smiles>CN1CCN(C)C(=Nc2ccc([N+](=O)[O-])cc2C(=O)Nc2ccccc2)C1</smiles>

B

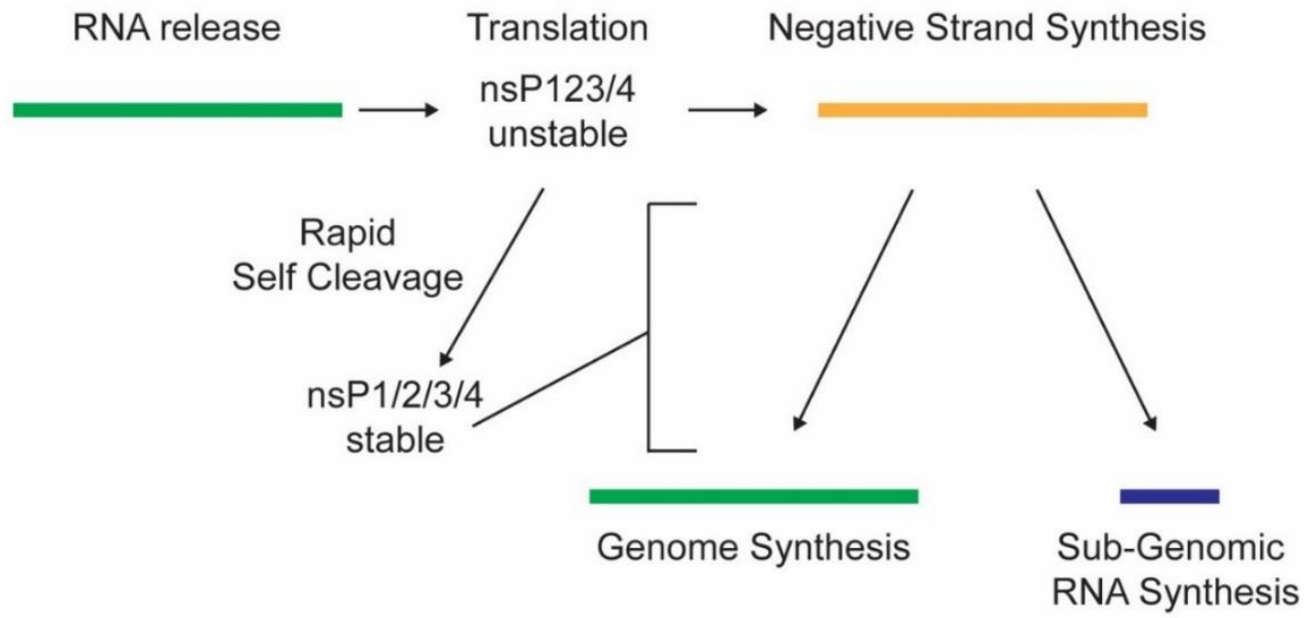

Figure 6. Summary of VEEV replication A) The structure of the VEEV inhibitory amidine compound, ML336, as has been previously reported. B) A summary of the RNA replication process of alphaviruses. First, the genomic RNA (green line) is released from the virion into the cytoplasm, where it can recruit ribosomes and be translated into the initial polyprotein, nsP123/4. This short-lived, initial polyprotein then synthesizes negative-sense RNA template strands (yellow line) from the positive-sense genome. The polyprotein then undergoes self-cleavage to form the mature, stable, replicase complex, nsP1/2/3/4, which then synthesizes additional genomic RNA (49S) as well as subgenomic RNA (26S, blue line). 
In order to characterize the potential of viral populations to gain resistance to these antiviral treatments, experiments were performed that isolated resistant virus from the treated population. The locations of mutations granting resistance were then determined using sequencing, which can provide information on both potential mechanisms of compound action as well as potential sites of interaction between viral proteins and the compounds. Resistance mutations to the hit quinazolinone compound and benzamidine ML336 both map to two regions; 1) the N-terminal region of nsP2, nSP2 mutations $\mathrm{Y} 102 \mathrm{C}, \mathrm{D} 116 \mathrm{~N}, \mathrm{E} 11 \mathrm{VV}, \mathrm{E} 118 \mathrm{~V}$, and 2) the $\mathrm{N}$-terminal region of $\mathrm{nsP} 4$, mutation Q210 in $\mathrm{nsP} 4_{\mathrm{Q} 210 \mathrm{R}}{ }^{58,85,88,96,127,130,162}$. The ML336 resistance mutations in nsP2 were clustered in a region that has recently been shown to be part of the so-called "stalk" domain of the helicase domain ${ }^{98}$. An X-ray crystal structure of CHIKV nsP2 helicase domain shows that the nsP2 ${ }_{1102, D 116, E 117}$, and ${ }_{\mathrm{E} 118}$ residues are not associated with the active site or RNA recognition domains of the enzyme. Rather, these residues are within the distal part of a large alpha helix and flanked by smaller disordered regions. The resistance mutations in nsP4 are also upstream of the active site of the RdRP, in a region with no known functional activity ${ }^{125}$. Previous work in Sindbis virus indicates that the $\mathrm{N}$-terminal region of nsP4 has importance for viral RNA synthesis possibly in a protein-to-protein interaction dependent manner; however, no clear functions have been understood ${ }^{163}$.

In this study we sought to understand how ML336 and its benzamidine analogues inhibit VEEV replication with a hypothesis that ML336 is directly-acting on the replicase complex and interferes with viral RNA synthesis. Our results confirm that viral RNA synthesis is efficiently abrogated by ML336, an activity mediated through the viral replicase complex. We found that the synthesis of all species of viral RNAs (i.e., the genomic (49S), subgenomic (24S) positive-sense RNA, and negative sense RNA) are inhibited by ML336, and that the RNA inhibition displayed by ML336 is maintained in a 
cell free system of viral RNA synthesis. These findings suggest that the primary antiviral activity of ML336 is the inhibition of viral RNA synthesis during infection.

\section{Materials and Methods}

\section{Cell culture and viral strains}

Baby hamster kidney (BHK) clone 21 cells (ATCC CCL-10) and Vero 76 (African Green monkey kidney fibroblasts) (ATCC® $\mathrm{CRL}-1587^{\mathrm{TM}}$ ) were maintained in Modified Eagle's Medium with Earle's Balanced Salt Solution and L-glutamine (MEM-E, Corning 10-010-CV) supplemented with 10\% fetal bovine serum (FBS) (Corning 35-011-CV). Cells were maintained at $37^{\circ} \mathrm{C}$ in humidified incubators with $5 \% \mathrm{CO}_{2}$. VEEV strain TC-83 (gift of Dr. Connie Schmaljohn, USAMRIID) was used for this study. The strain V3526 was generated from a plasmid as described previously (Chung et al., 2014). Infections were carried out using a virus infection medium (Modified Eagle's Medium with Earle's Balanced Salt Solution supplemented (Corning 15-010-CV) with 1x GlutaMAX (Gibco 35050061), 25 mM HEPES (Corning 25-060-CI), and 10\% FBS. For Chikungunya virus (CHIKV) experiments, CHIKV strain 181/25 (BEI Resources NR-13222) was used. V3526 experiments were carried out in an infection media that contained L-glutamine instead of GlutaMAX (Modified Eagle's Medium with Earle's Balanced Salt Solution with L-glutamine (Corning 10-010-CV), 25 mM HEPES, 10\% FBS)

\section{Immunoblot analysis}

Cells were lysed using Laemmli buffer 4\% SDS (RPI L22010) w/v, 20\% glycerol w/v, $120 \mathrm{mM}$ Tris- $\mathrm{HCl} \mathrm{pH}=6.8$, bromophenol blue) and $42 \mu \mathrm{M}$ of dithiothreitol (DTT Enzo: ALX-280-001-G025)) and the homogenate was passed through a needle to shear the DNA, with additional sonication as necessary. After denaturation at $100^{\circ} \mathrm{C}$ for ten 
minutes, samples were loaded onto a gradient gel (4-20\%, GenScript, M42012) and run at $140 \mathrm{~V}$ for 90 minutes in $1 \mathrm{X}$ MOPS-SDS buffer (GenScript, M00138). Proteins were transferred to a PVDF membrane (Bio-Rad 1620177). The membrane was blocked with $5 \%$ dry milk in TBS-T, and probed using antibodies for the indicated proteins. Anti-nsP2 (Clone no. 8A4B3, available from Kerafast, EUL015) was generated as a custom mouse monoclonal antibody from GenScript using bacterially expressed recombinant nsP2 protein, used at a final concentration of $0.4 \mu \mathrm{g} / \mathrm{mL}$. Beta-actin was detected using an antibody directly linked to horse radish peroxidase (HRP) (Cell Signal 8H10D10) at a dilution of 1:1,000. Anti-nsP2 was detected using (HRP)-conjugated rabbit anti-mouse IgG antibody (Sigma A0168) final concentration 1:10,000. Images were developed using ECL reagent and captured using an Azure Biosystems c300 imaging system.

\section{Analysis of viral RNA synthesis in vivo by metabolic labelling with ${ }^{3} \mathrm{H}$-uridine}

Cells were infected with VEEV at a multiplicity of infection (MOI) of ten on ice for one hour; and afterwards, cells were washed with ice-cold 1X phosphate buffered saline (PBS Caisson Labs PBL07) and transferred to a $37^{\circ} \mathrm{C} \mathrm{CO}_{2}$ incubator to initiate the replication $(\mathrm{T}=0)$. At 6 hours post-infection $(\mathrm{HPI})$, cells were washed and pulsed with virus infection media containing actinomycin D (act D) (1 $\mu \mathrm{g} / \mathrm{mL}$, Sigma Aldrich A9415), tritium-labelled uridine $\left({ }^{3} \mathrm{HU}\right)(5 \mu \mathrm{Ci} / \mathrm{mL}$, Perkin Elmer NET367250), and ML336 at various concentration for two hours. Total RNA was isolated from the cells using RNAzol RT according to manufacturer's instructions (Molecular Research Center RN190). Total RNA was mixed with $10 \mathrm{~mL}$ liquid scintillation cocktail (BETA BLEND, MP Biochemicals 0188245004) and the radioactivity was measured using a Perking Elmer Tri-Carb 2910 TR liquid scintillation counter. For CHIKV, infection proceeded to $8 \mathrm{HPI}$ for ${ }^{3} \mathrm{HU}$ pulse labelling. For compound treatment, ML336 dissolved in DMSO (Sigma Aldrich D8418) was added in the ${ }^{3} \mathrm{HU}$ labelling mixture with a final DMSO concentration of $0.25 \%$. 
ML336 concentrations are indicated in the results section for specific experiments. For cycloheximide (CHX) (Sigma Aldrich C7698) treatment, cells were treated with ${ }^{3} \mathrm{HU}$ labelling mixture containing $\mathrm{CHX}$ with a final concentration of $8.8 \mu \mathrm{g} / \mathrm{mL}$.

\section{Fluorography}

250,000 cells were infected at an MOI of 20 with VEEV TC-83. Cells were pulsed for the indicated times post infection with $1 \mu \mathrm{g} / \mathrm{mL}$ act $\mathrm{D}, 40 \mu \mathrm{Ci} / \mathrm{mL}{ }^{3} \mathrm{HU}$ and $2.5 \mu \mathrm{M}$ ML336. RNA was isolated using RNAzol RT. One $\mu \mathrm{g}$ RNA was used per treatment for the fluorgram. Fluorography was performed following a protocol published by John Aris $^{164}$.

\section{RNA labeling of V3526 and mutant viruses}

Cells were infected with VEEV V3526 at an MOI of 5 and pulsed in 2 hour increments up to 14 hours. Later time points were also collected at 18, 24, 30, and 40 HPI. Peak RNA synthesis was found to occur between 14 and $18 \mathrm{HPI}$ (data not shown). To compare the effectiveness of the compound, cells were infected at an $\mathrm{MOI}$ of 3 and allowed to proceed to $13 \mathrm{HPI}$ before pulsing with $1 \mu \mathrm{M}$ ML336 or an equivalent volume of DMSO. RNA was collected and radioactivity was detected as described above.

\section{Strand-specific quantitative real-time PCR (qRT-PCR) of VEEV RNA}

Detection of positive and negative sense, genomic viral RNA was carried out using a strand-specific qRT-PCR method adapted from Plaskon et al ${ }^{165}$. Briefly, cDNA was generated using tagged primers for detecting positive-sense and negative-sense RNA. The generated cDNA was then used in qRT-PCR using TaqMan chemistry (Applied Biosystems TaqMan gene expression master mix ThermoFisher 4369016) with a strand-specific primer set. A fluorescent probe was used for both analyses. The primer sequences are given in the following table. Lowercase sequences are additional 
sequence added for primer identification, sequences in italics are specific for viral RNA, and sequences in bold were used to identify only those cDNA sequences that were produced due to primer binding. All primers and probes were custom generated by IDT.

\begin{tabular}{|l|l|}
\hline Primer & Sequence \\
\hline nsP1 positive & GGCAGTATCGTGAATTCGATGCCTGACCTGGAAACTGAGACTATG \\
Tag & \\
\hline nsP1 negative & GGCAGTATCGTGAATTCGATGC GGCGACTCTAACTCCCTTATTG \\
Tag & \\
\hline nsP1 positive & aataaatcataa CTG ACC TGG AAA CTG AGA CTA TG \\
FWD & \\
\hline nsP1 positive & aataaatcataa GGC AGT ATC GTG AAT TCG ATG C \\
REV & \\
\hline nsP1 negative & aataaatcataa GGC GAC TCT AAC TCC CTT ATT G \\
FWD & \\
\hline nsP1 negative & aataaatcataa GGC AGT ATC GTG AAT TCG ATG C \\
REV & \\
\hline nsP1 probe & /56-FAM/TCC GTC AAC /ZEN/CGC GTA TAC ATC CTG /3IABkFQ \\
\hline
\end{tabular}

\section{Enrichment of viral replicase complexes from infected cells}

VEEV replicase complexes were isolated according to the protocol published by Barton et $\mathrm{al}^{166}$. Cells were infected with VEEV TC-83 at $10 \mathrm{MOI}$ and incubated for 6 hours. Then, cells were washed with ice-cold, sterile PBS and the cells were incubated in hypotonic RS buffer (10 mM NaCl, $10 \mathrm{mM}$ Tris- $\mathrm{HCl}$, pH7.8) supplemented with Protease inhibitor cocktail III, $30 \mu \mathrm{L}$ per $20 \times 10^{6}$ cells, (Research Products International P50700-1) on ice for 15 minutes. Cells were scraped into buffer and thoroughly homogenized using a Dounce homogenizer. Nuclei were removed by centrifugation at $900 \mathrm{xg}$ for 10 minutes at $4^{\circ} \mathrm{C}$. Supernatant containing the cytoplasmic fraction was transferred to microcentrifuge tubes and centrifuged at $15,000 \times \mathrm{g}$ for 20 minutes at $4{ }^{\circ} \mathrm{C}$. The supernatant (S15 fraction) was removed and pellets (P15 fraction) were suspended in RS buffer supplemented to $15 \%$ glycerol for storage at $-80^{\circ} \mathrm{C}$.

\section{In vitro viral RNA synthesis assay}


VEEV viral RNA synthesis assay was adapted from Barton et al ${ }^{166}$. Ten microliters of P15 fraction enriched for VEEV viral replicase complexes, which is equivalent of approximately $1.25 \times 10^{6}$ infected cells, was combined with a same volume of a RNA synthesis mix (100 mM Tris- $\mathrm{HCl} \mathrm{pH} 7.8,100 \mathrm{mM} \mathrm{KCl}, 20 \mu \mathrm{g} / \mathrm{mL}$ act D, $20 \mathrm{mM}$ DTT, $10 \mathrm{mM}$ creatine phosphate, $50 \mu \mathrm{g} /$ reaction creatine phosphokinase, $4 \mathrm{mM}$ of ATP, GTP, and UTP, $20 \mu \mathrm{M}$ CTP, $12 \mathrm{mM} \mathrm{MgCl}_{2}$ ) (nucleotides from NEB N0450S) on ice and 1 $\mu \mathrm{L}$ of SUPERaseln RNase inhibitor (Ambion AM2694), $5 \mu \mathrm{g}$ of yeast tRNA (Ambion 15401011), and $5 \mu \mathrm{Ci}$ of [ $\left.\alpha^{-33} \mathrm{P}\right]-\mathrm{CTP}$ (Perkin-Elmer NEG608H) were added per reaction. After an incubation at $37^{\circ} \mathrm{C}$ for 90 minutes, RNA was isolated from each reaction using RNAzol RT and RNA mini prep kit columns (Zymo Research R2052) according to manufacturer's instructions, with an additional wash step before elution. For compound addition, ML336 was added to reaction mixtures before incubation at $37^{\circ} \mathrm{C}$ at the indicated concentrations. The final DMSO concentration was $0.25 \%$.

\section{Autoradiography of viral RNA}

After extraction of viral RNA from the in vitro reactions, the RNA was mixed 1:1 with a glyoxal loading buffer/dye with ethidium bromide (Ambion AM8551) and denatured at $50{ }^{\circ} \mathrm{C}$ for 30 minutes. Samples were chilled briefly and loaded for RNA electrophoresis. RNA electrophoresis was performed through a denaturing agarose gel containing 0.8\% agarose, 1X MOPS (Quality Biological 351-059-10), and 2.2 M formaldehyde. Electrophoresis was performed at $60 \mathrm{~V}$ for 70 minutes, then the gels were rinsed in nuclease-free water once and treated with $0.1 \mathrm{~N} \mathrm{NaOH}$ for 40 minutes at room temperature with continuous rocking. Gels were neutralized in 20X saline-sodium citrate (SSC) buffer ( $3 \mathrm{M} \mathrm{NaCl}, 300 \mathrm{mM}$ trisodium citrate, $\mathrm{pH} 7.0$ ) for 40 minutes. RNA was transferred to a neutral nylon membrane (GE Nytran 10416296). The RNA on the membrane was then UV cross-linked for 5 minutes at $4 \mathrm{~mW} / \mathrm{cm}^{2}$. Autoradiograms were 
developed using a phosphor screen (Kodak K screen 1707843) and documented using a Pharos FX plus (BioRad). Densitometry analysis was performed using Fiji image analysis software ${ }^{167}$.

\section{Cell-based anti-VEEV assay}

Anti-VEEV activity of compounds was measured using a cell-based CPE assay as previously described ${ }^{158}$. Briefly, Vero 76 cells seeded in a 96 well plate were infected with VEEV TC-83 at an MOI of 0.05 in the presence of test compounds, highest concentration of $50 \mu \mathrm{M}$, serially diluted $1: 2$ with a total of eight concentrations, lowest concentration $400 \mathrm{nM}$. Infected cells were incubated for $48 \mathrm{~h}$ and cell viability, protection from VEEV-induced CPE, was measured using CellTiter-Glo (Promega G7570). EC 50 was calculated with a 4-parameter logistic model (XLfit, IBDS).

\section{Statistics}

Statistics were performed in GraphPad Prism version 9. Unless otherwise indicated significance was calculated using ANOVA with Dunnett corrections for multiple comparisons. All graphs use the mean value, with error reported as standard deviation.

\section{Results}

\section{VEEV viral RNA production peaks at 6-8 hours post infection.}

To determine the optimal timepoint to examine the inhibitory effects of ML336 on viral RNA synthesis, we first measured the rate of VEEV viral RNA synthesis over the course of infection using metabolic labeling of RNA. After BHK21 cells were infected with VEEV TC-83 at an MOI of 10, the synthesis of viral RNA was tracked in two-hour increments beginning at $2 \mathrm{HPI}$ by pulsing with $3 \mathrm{HU}$ in the presence of act $\mathrm{D}$, which 
allowed us to detect only the synthesis of viral RNA by inhibiting cellular RNA transcription from DNA templates. Using this assay, we found that VEEV RNA synthesis was detectable at $2 \mathrm{HPI}$, and continued until $18 \mathrm{HPI}$ Figure $7 \mathrm{~A}$. VEEV RNA synthesis reached its peak between 6 and $8 \mathrm{HPI}$ and began to decrease at $10 \mathrm{HPI}$. There is no significant difference between the three pulses from 4-10 HPI. So any of the three pulses should allow for strong detection of viral RNA synthesis. The 6-8 HPI pulse was selected due to a balance of length of the experiment, as well as the higher magnitude displayed in this assay.

We measured expression of nsP2 as a marker for the viral replicase complex over the course of infection by immunoblotting. Detectable amounts of nsP2 were detected at $4 \mathrm{HPI}$, and the expression peaked at $8 \mathrm{HPI}$, which is consistent with the timeline of viral RNA synthesis Figure 7 B. Later time points were selected due to previous knowledge about the kinetics of nsP2 in our lab. These data together indicate that the greatest level of RNA synthesis of VEEV is from 6-8 HPI. Based on this result, we chose the 6-8 HPI time point for subsequent experiments for viral RNA synthesis assays. 


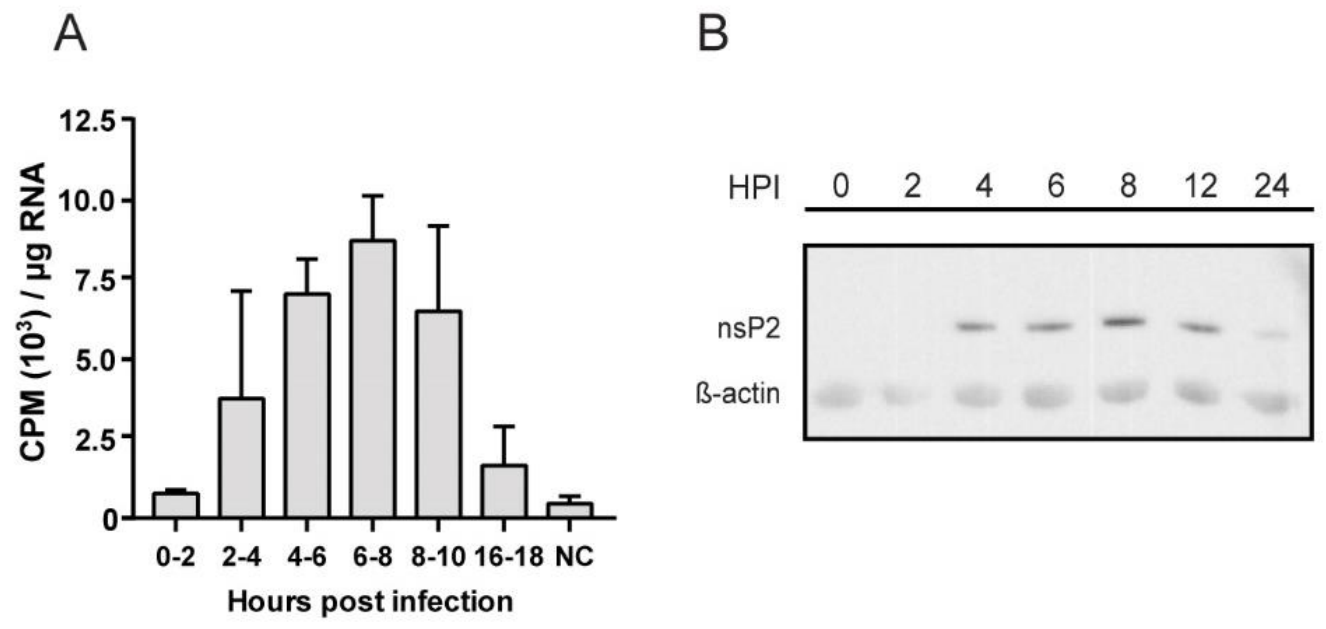

Figure 7. VEEV RNA synthesis peaks at 6-8 $\mathrm{HPI}$.

A) BHK 21 cells were infected with VEEV as described in the Materials and Methods of this chapter and treated with act D and 3HU to selectively label newly synthesized viral RNA. Total RNA was isolated and subjected to liquid scintillation counting. The amount of label detected is reported as counts per minute (CPM) per microgram of isolated RNA. NC is an uninfected negative control collected at 18 hours. Bars represent two biological replicates from one representative experiment of two. Error bars indicate standard deviation. B) BHK 21 cells were infected with VEEV and total cell lysate was used for immunoblotting to detect nsP2. Beta-actin is included as a protein loading control. Image is from one representative experiment of three. 


\section{Antiviral activities of ML336 and derivatives correlated with their inhibition of viral RNA synthesis.}

Based on our previous findings ${ }^{127}$, we hypothesized that the primary antiviral mechanism of the benzamidine scaffold, including ML336, is inhibition of viral RNA synthesis. To test this hypothesis, we chose to examine the RNA synthesis inhibition activities of an array of structurally analogous compounds (Table 1) based around the ML336 amidine scaffold Figure $8^{159}$. Multiple compounds have been developed using the base structure of ML336, with the pursuit of further improvements to both efficacy and pharmacokinetics, this optimization is pursued for every potential lead therapeutic compound that is discovered through the high throughput screening process. Each of these compounds has been found to be variously effective at inhibiting VEEV induced CPE. If the efficacy of this range of compounds at inhibiting VEEV induced CPE and RNA synthesis corelate, it indicates that RNA synthesis inhibition is a feature of the more general benzamidine scaffold and not unique to ML336. This also strengthens the hypothesis that RNA synthesis inhibition is a major contributor to the antiviral activity of the benzamidine compound family. ML416, which has an antiviral mechanism that is independent of viral RNA synthesis and is structurally distinct, was used as a control for

comparison $^{146}$. We also included our initial hit compound from our high throughput study, CID15997213 ${ }^{127}$.

Nine compounds with $50 \%$ cytopathic effect (CPE) inhibitory concentrations $\left(\mathrm{EC}_{50-\mathrm{CPE}}\right)$ ranging from $0.1 \mu \mathrm{M}$ to greater than $50 \mu \mathrm{M}$ were tested in the $3 \mathrm{HU}$ labeling assay ${ }^{159}$. Cells infected with VEEV TC-83 at an MOI of 10 were treated with each compound at $1 \mu \mathrm{M}$ at six $\mathrm{HPI}$ and their RNA synthesis inhibitory activities were compared with their $\mathrm{EC}_{50-\mathrm{CPE}}$. The concentration was held constant instead for example 
using the $\mathrm{IC}_{50}$ of each compound so that each compounds efficacy could be directly compared. Using the middle efficacious concentration of each compounds would not allow for variation between the measurements, and the correlation could not be determined. Each activity was quantified as percent inhibition compared to the DMSOtreated control. While ML416 did not show any RNA synthesis inhibitory activity as expected, each of the compounds showed various amounts of reduction of viral RNA synthesis Figure 8. Importantly, we found a trend that compounds that were more potent in the CPE-bases assay were generally more efficacious as inhibiting RNA synthesis, $R^{2}=0.42 p=0.059$, as measured by the incorporation of ${ }^{3} \mathrm{H}$-uridine into viral RNA. This experiment was performed from $6-8 \mathrm{HPI}$ as this was found to be the time of maximal RNA synthesis by the virus. At this time post infection, and at the MOI that was used, the virus has already entered the cell and performed the activities of early infection (i.e. receptor binding, endocytosis, release of the viral RNA into the cytoplasm) and the initial translation and processing of the nsP polyprotein. Thus, the reduction in viral RNA synthesis that is seen to occur after treatment with these compounds is likely to occur independently from these other early infection processes. This indicates that the antiRNA synthesis activity of the benzamidine family is likely mediating the antiviral effects that that occur during infection with VEEV.

Several compounds that were tested in the assay were strongly inhibitory of RNA synthesis but had little effect on cell viability, such as CMP3. While none of these compounds were cytotoxic, $\mathrm{CC}_{50}>50 \mu \mathrm{M}^{159}$, it is possible there are some off target effects that make the compounds less protective. 


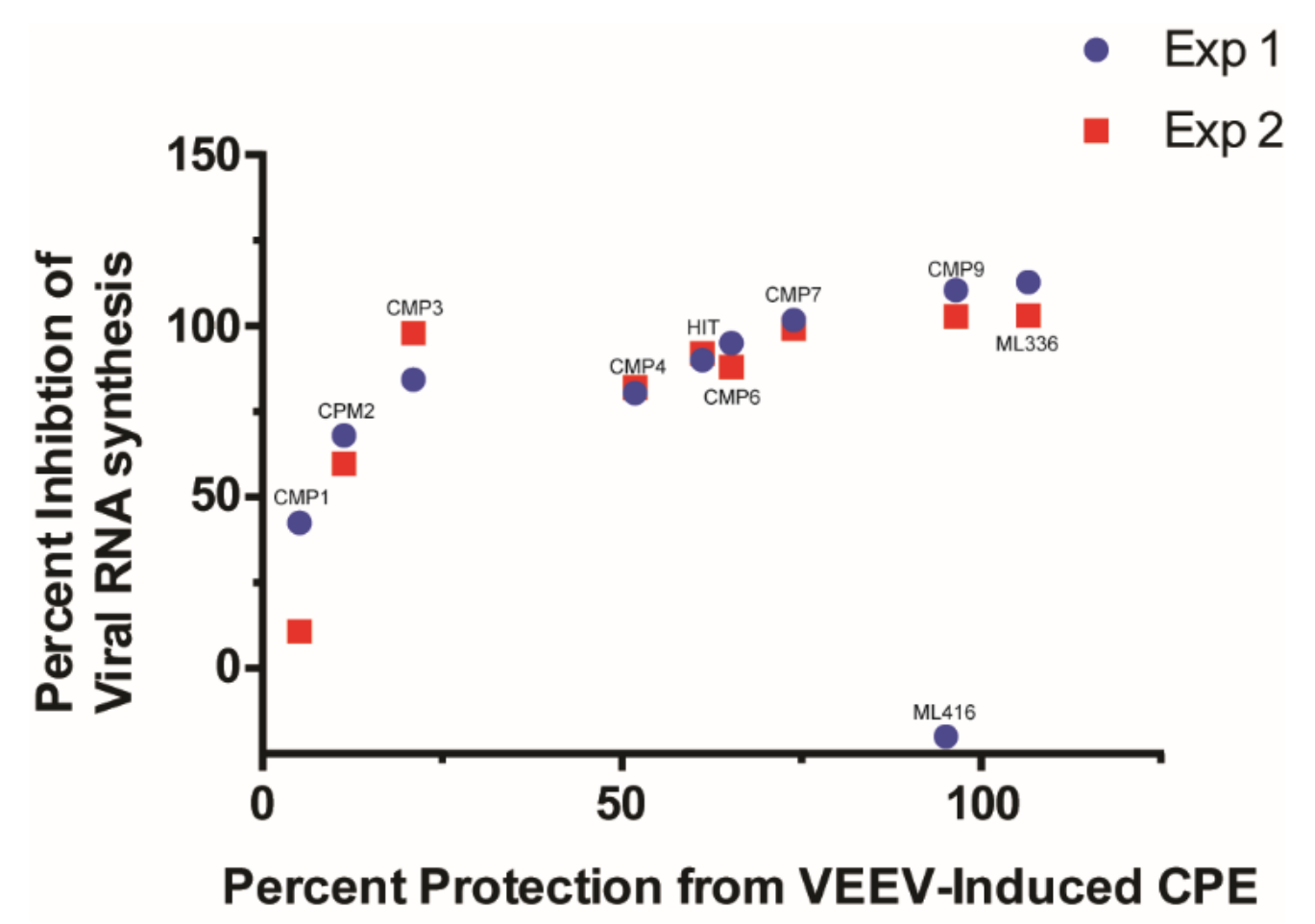

Figure 8. Compounds derived from ML336 also inhibit viral RNA replication.

BHK 21 cells were infected with VEEV as described and infection proceeded to $6 \mathrm{HPI}$. Cells were pulsed for 2 hours with actD, ${ }^{3} \mathrm{HU}$, and one of the compounds of interest at 1 $\mu \mathrm{M}$ or DMSO vehicle control. The amount of ${ }^{3} \mathrm{HU}$ incorporated into the viral RNA was quantified by liquid scintillation counting. The percentage of label that was incorporated compared to a DMSO control, with $100 \%$ inclusion resulting in $0 \%$ inhibition, and $0 \%$ inclusion resulting in $100 \%$ inhibition. This data was compared to the percent protection from TC-83-induced CPE in a cell-based assay. Percent protection is the percent of viable cells after infection compared to DMSO vehicle control treatment. $R^{2}=0.42$ $\mathrm{p}=0.059$, calculated using linear regression in GraphPad Prism. ML416 was included as 
an outgroup, as it is effective at inhibiting VEEV induced CPE but is known to function by an RNA synthesis independent mechanism. Each point represents three biological replicates and the experiment was repeated twice (Exp 1 and Exp2). Compound IDs are found in Table 2. 


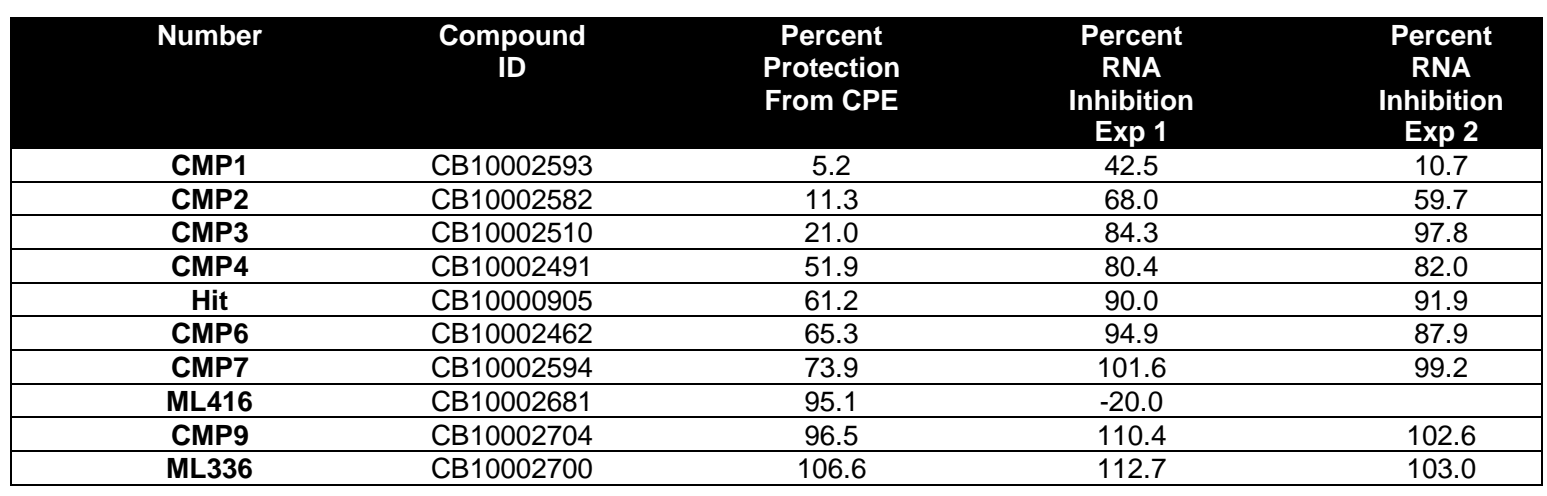

Table 2. Chemical IDs and effectiveness in the CPE and RNA inhibition assays.

Compound ID numbers are the same as the structure IDs available in PubChem. 


\begin{tabular}{|c|c|c|}
\hline Number & Compound ID & Structure \\
\hline CMP1 & CB10002593 & \\
\hline CMP2 & CB10002582 & \\
\hline CMP3 & CB10002510 & \\
\hline CMP4 & CB10002491 & \\
\hline Hit & CB10000905 & \\
\hline CMP6 & CB10002462 & \\
\hline CMP7 & CB10002594 & \\
\hline
\end{tabular}




\begin{tabular}{|l|l|l|}
\hline ML416 & CB10002681 & \\
\hline CMP9 & CB10002704 & \\
& & \\
\hline ML336 & CB10002700 & \\
& & \\
\hline
\end{tabular}

Table 3: Structures of the selected compounds 


\section{The anti-RNA synthesis activity of ML336 is VEEV-specific and has no effect on cellular RNA synthesis.}

Having determined that ML336 and related compounds are able to inhibit VEEV RNA synthesis during active replication, we sought to further characterize this anti-RNA synthesis activity. First, we determined the potency of ML336 against VEEV viral RNA synthesis. Cells were infected with VEEV strain TC-83 and treated with $3 \mathrm{HU}$ and various concentrations of ML336 from six to eight HPI as described in the Materials and Methods. The amount of $3 \mathrm{HU}$ incorporated into the total isolated RNA was measured and a dose response curve was generated to calculate the $\mathrm{IC}_{50}$ Figure 9 A. ML336 showed strong, dose-dependent inhibition of viral RNA synthesis activity with an $\mathrm{IC}_{50}$ of $1.1 \mathrm{nM}$ with a standard deviation of $0.7 \mathrm{nM}$. At $40 \mathrm{nM}, \mathrm{ML} 336$ decreased viral RNA synthesis to $7 \%$ of the control. These data indicate that VEEV RNA synthesis is efficiently inhibited by ML336. Also, ML336 was efficacious at inhibiting viral RNA synthesis at the $\mathrm{EC}_{50}$ determined by the cell-based assay.

Previously, we determined that compounds based around the ML336 benzamidine scaffold had no antiviral effect on CHIKV in cell culture using the CPE assay $^{145}$. If the antiviral effect of this compound is mediated by the inhibition of viral RNA synthesis, then CHIKV will also resist this activity. We measured the inhibition of RNA synthesis by ML336 treatment on CHIKV using the $3 \mathrm{HU}$ assay. As can be seen in Figure $9 \mathrm{~B}, \mathrm{ML336}$ had no effect on CHIKV RNA synthesis even at $4 \mu \mathrm{M}(P>0.22$, ANOVA), the highest concentration we tested, and 4000 -fold higher than the $\mathrm{IC}_{50}$ value of the compound against VEEV in this assay, compared to an untreated positive control.

To measure the effect of ML336 on cellular RNA production, uninfected BHK cells were incubated with ML336 at the indicated concentrations or with a DMSO control in the presence of $3 \mathrm{HU}$ without act D Figure $9 \mathrm{C}$. Overall, ML336 did not show an 
inhibitory effect on cellular RNA synthesis. A small decrease in cellular RNA synthesis was detected only at the highest concentrations of ML336, $50 \mu \mathrm{M}$ and $25 \mu \mathrm{M}$, which are over 20,000 -fold higher than the $\mathrm{IC}_{50}$ value, indicating that the inhibitory activity of ML336 against cellular RNA synthesis is negligible at working concentrations. 
A

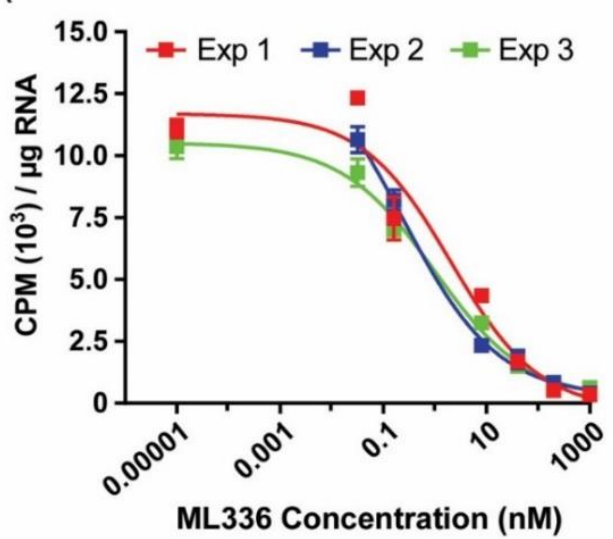

B

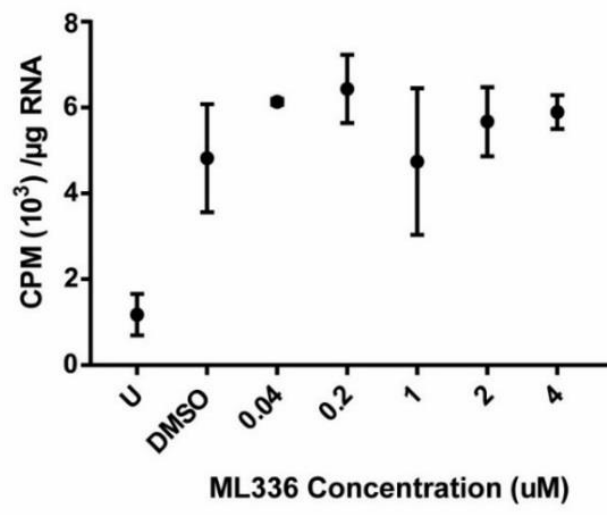

C

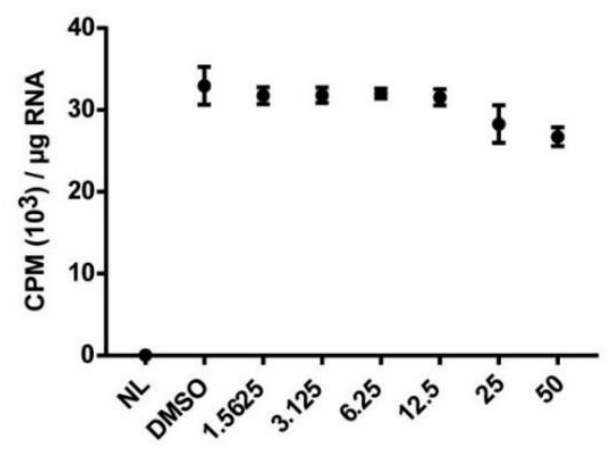

ML336 Concentration (uM)

Figure 9. The RNA synthesis inhibition of ML336 is highly specific and has no effect on cellular RNA synthesis.

A) BHK 21 cells were infected with VEEV as described and cells were pulsed from 6-8 hours post infection with act D, 3HU, and ML336 at the indicated concentrations. Each point represents three biological replicates, error was calculated as the standard deviation. Dose-response curves were generated using four parameter curve fitting in Graph Pad Prism software $9^{\text {th }}$ edition. B) BHK 21 cells were infected with CHIKV at an $\mathrm{MOI}$ of 10 and infection proceeded as for VEEV. Cells were treated with the indicated 
amounts of ML336. $U$ is an uninfected control. Graph is of one representative experiment of two total experiments, each point represents three biological replicates, and error is standard deviation. C) Uninfected BHK 21 cells were treated with VEEV at the indicated concentrations or DMSO control. Cells were pulsed for 2 hours with compound and $3 \mathrm{HU}$. NL is an unlabeled control reaction. Graph is of a single representative experiment of two experiments, points each represent three biological replicates, and error is standard deviation. 


\section{Viral isolates that can replicate in the presence of ML336 resist the RNA synthesis of inhibition of the compound.}

As previously mentioned, we have isolated several strains of VEEV that resist treatment by either our initial hit compound ${ }^{127}$ or ML336, by serially passaging in the presence of the compound or by direct sequencing in the presence of the compound. These resistant viruses have mutations mapped in the N-terminal regions of nsP2 (e.g., $\mathrm{nsP} 2_{\mathrm{Y} 102 \mathrm{C}}$ mutation) or of $\mathrm{nsP} 4$ (e.g., $\mathrm{nsP} 4_{\mathrm{Q} 210 \mathrm{~K}}$ mutation) ${ }^{127}$. Either of these mutations is enough to cause resistance in isolation, i.e. only one of the two mutations is required for compound resistance. We hypothesized that if inhibition of viral RNA synthesis is the primary outcome of ML336 treatment, then these mutants would maintain levels of RNA synthesis similar to the parental virus in the $3 \mathrm{HU}$ incorporation assay. We introduced the mutations that were found in the mutant strains into the plasmid encoding the full, infectious genome of V3526 and then produced the mutant virus strains in the presence of the initial hit compound at a concentration of $5 \mu \mathrm{M}^{127}$. After determining that maximum levels of RNA synthesis occurred at $14 \mathrm{HPI}$ (data not shown) we performed a metabolic labeling experiment in the same manner as described in our methods.

As expected, viral RNA synthesis of the parental V3526 was sensitive to treatment with ML336, and viral RNA synthesis was fully abrogated at $5 \mu \mathrm{M}$ Figure 10. Comparatively, the mutant viruses showed varying levels of sensitivity to ML336. The nsP2 ${ }_{\text {Y102C }}$ mutant showed $76.2 \%$ RNA synthesis compared to the control and nsP4 $4_{\mathrm{Q} 210 \mathrm{~K}}$ mutant showed no inhibition compared to a vehicle control. This result clearly shows that the compound-resistant mutant viruses are able to overcome the RNA synthesis inhibitory effects of ML336. Our data so far support the hypothesis that ML336 is a viral RNA synthesis inhibitor that acts through nsP2 and nsP4. 
DMSO

ML336

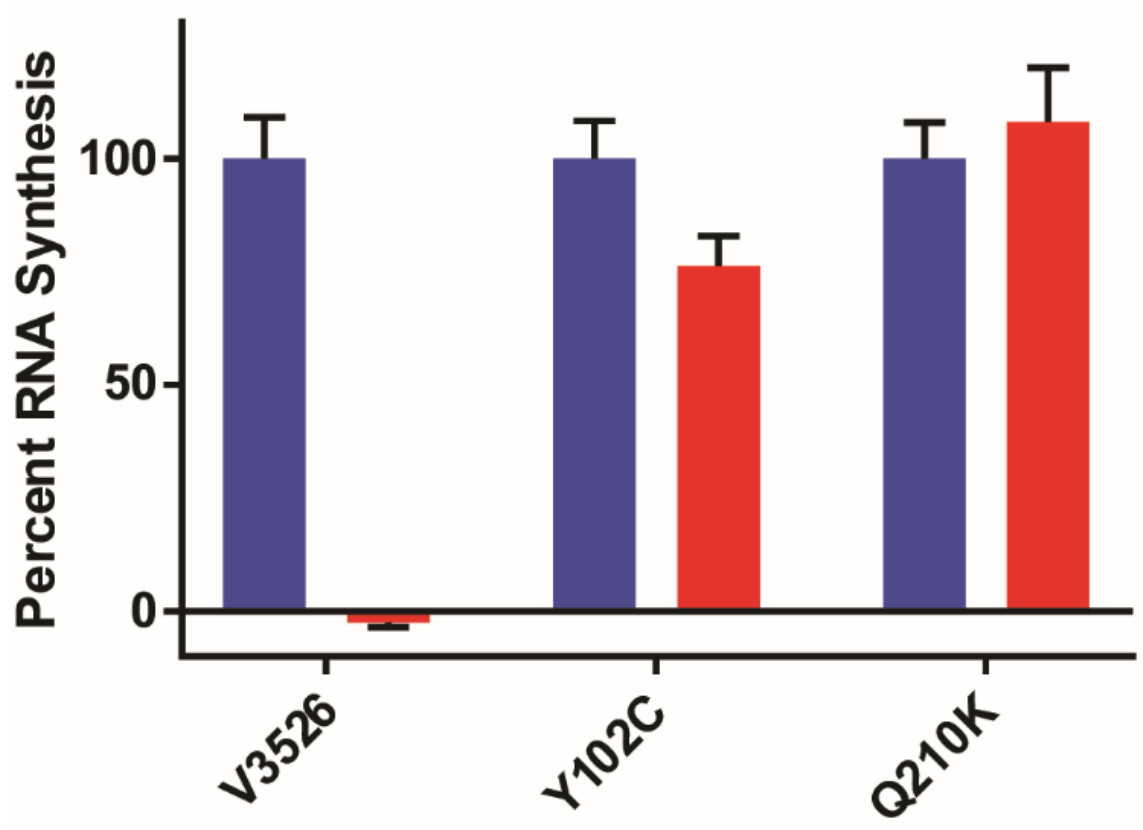

Figure 10. ML336 resistant mutations grant resistance to RNA synthesis inhibition. Mutations were introduced to the clonal VEEV V3526 backbone and these mutant viruses were subjected to the RNA synthesis inhibition assay as described at a concentration of $5 \mu \mathrm{M}$ ML336. The amount of $3 \mathrm{HU}$ label was quantified by liquid scintillation counting. The amount of incorporated label was divided by the amount present in the DMSO treated control and multiplied by 100 to yield the percent of RNA synthesis. Graph is from data from two experiments, each with three biological replicates. Error is reported as standard deviation. 


\section{ML336 inhibits both positive and negative RNA synthesis of VEEV.}

During infection with VEEV, several RNA species are generated by the virus which have distinct functions. We next sought to determine if ML336 specifically targeted the synthesis of one or more of these RNA species. To determine if ML336 inhibits either positive or negative-sense RNA production, we used a strand-specific qRT-PCR ${ }^{165}$. VEEV-infected cells were treated either with ML336 or DMSO at $4 \mathrm{HPI}$, and the copy numbers of positive and negative-sense viral RNA were quantified and compared. This earlier time point was selected as the rate of negative-sense RNA production decreases later in infection. As shown in Figure $11 \mathrm{~A}$, the control group (DMSO-treated) had almost a 1 log increase in the amount of positive-sense RNA and an approximately half log increase of negative-sense RNA during the period of 4 to $6 \mathrm{HPI}$.

In the presence of ML336, however, the amount of positive- and negative-sense viral RNA did not increase at all, which demonstrated the inhibition of both positive- and negative-sense viral RNA synthesis by ML336. The presence of ML336 did not affect the ratio of positive to negative-sense RNA. The copy number of positive-sense RNA was 10,000 -fold higher than that of the negative-sense RNA at all time points and in both ML336 treated and untreated conditions. This difference in the levels of the RNA species is consistent with findings previously reported in alphaviruses by others ${ }^{168}$. These results show that the synthesis of both the positive and negative-sense strands of VEEV RNA was affected by ML336.

\section{ML336 inhibits the RNA synthesis by the mature replicase complex.}

For alphaviruses, the majority of viral RNA synthesized in the infected cells is positive-sense RNA (See Figure 11 A) and the synthesis of positive-sense RNA is 
dependent on the amount of negative-sense genomic RNA (Figure 5) ${ }^{85}$; therefore, the inhibition of negative-sense RNA synthesis would result in the inhibition of positivesense RNA synthesis. To determine if the inhibition of viral positive-sense RNA synthesis by ML336 is due to the inhibition of negative-sense RNA synthesis, we sought to test the effect of ML336 on positive-sense viral RNA synthesis alone.

The alphavirus negative-sense RNA is produced only by the newly translated, short-lived, polyprotein, nsP123/4 or nsP1/23/4 ${ }^{61,160}$. Therefore, it has been wellestablished that inhibition of translation (e.g., treatment with cyclohexamide) prevents the production of the negative-sense RNA by nsP123/4, which allows us to measure the positive-sense specific RNA synthesis by the pre-formed, mature, viral replicase complex ${ }^{85,169}$.

We measured viral positive-sense RNA synthesis using our 3HU labelling assay in the presence of cyclohexamide $(\mathrm{CHX})$, a translation inhibitor, and evaluated the effect of ML336, Figure $11 \mathrm{~B}$. Treatment with $\mathrm{CHX}$ showed no significant difference in the total viral RNA production compared to the vehicle control. This data indicates that the majority of viral RNA that was being synthesized at this time was positive-sense RNA, which is consistent with data reported by others ${ }^{85}$ as well as our real-time PCR experiments shown in Figure 11 A. Importantly, we found that the treatment of VEEVinfected cells with ML336 completely abrogated viral RNA production even in the presence of $\mathrm{CHX}$. This shows that ML336 inhibited the synthesis of viral RNA generated by the mature replicase complex (i.e., positive-sense RNA), and the inhibition of the negative-sense viral RNA synthesis is not required for ML336 to inhibit positive-sense viral RNA synthesis. 


\section{ML336 inhibits the synthesis of both genomic and subgenomic VEEV RNA.}

Having determined that ML336 inhibits the synthesis of positive-sense VEEV RNA, we sought to determine if the inhibition of the positive-sense RNA strand was specific to either the genomic or the subgenomic RNA. Alphaviruses synthesize two species of positive-sense RNAs, the genomic RNA (49S), which is packaged into progeny virions and is used for translation of the nonstructural polyprotein, and the subgenomic RNA (26S), which is responsible for the production of the structural genes. The expression of these two RNAs is controlled by the amount of polyprotein that has been synthesized, and the current cleavage state of the polyprotein ${ }^{61,103,160}$.

To understand whether the inhibition of viral RNA synthesis was specific to genomic (49S) or subgenomic (26S) viral RNA, we analyzed the viral RNAs that were produced in the presence or absence of ML336 using fluorography of 3HU-labeled viral RNA. As Figure $11 \mathrm{C}$ shows, the addition of ML336 to VEEV-infected cells at any time post-infection up to 8 hours completely abrogated synthesis of both genomic and subgenomic viral RNA. This indicates that addition of ML336 inhibits the synthesis of both 495 and $26 S$ viral RNA in cells. 
A

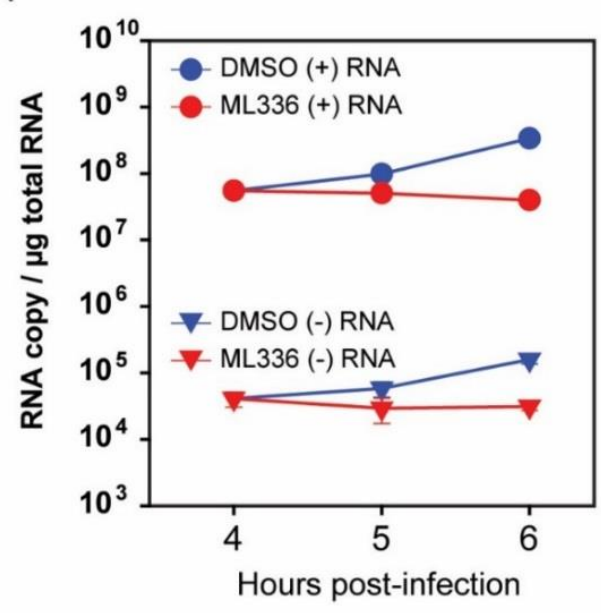

C

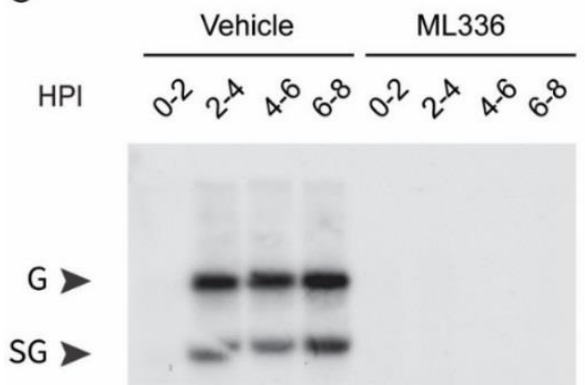

B

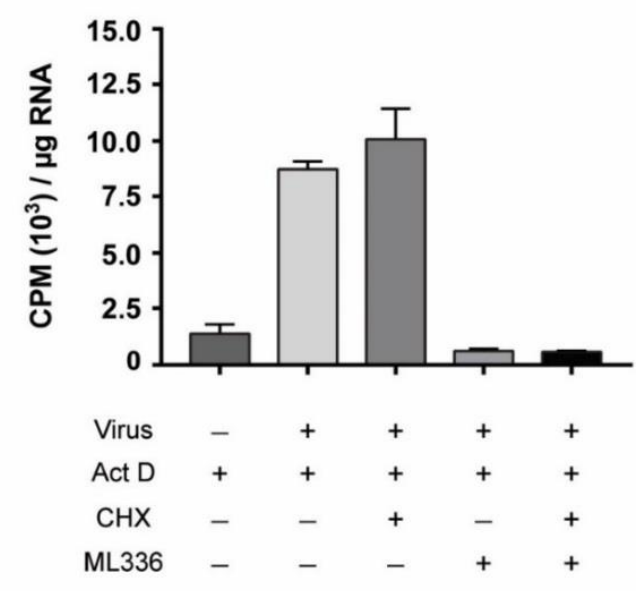

Figure 11. ML336 inhibits all stages of VEEV RNA synthesis.

A) Strand-specific qRT-PCR was performed on RNA isolated from TC-83 infected BHK 21 cells that were either treated with ML336 at $5 \mu \mathrm{M}$ or DMSO vehicle control. RNA copy number was measured by using a standard curve with known viral RNA copy numbers. Each point represents a mean and standard deviation from three biological replicates. B) Metabolic labeling of VEEV RNA was performed as before using act D and $3 \mathrm{HU}$, with data collected by liquid scintillation counting. Cells were labeled from 6-8 HPI. CHX: cyclohexamide. Graph is from one representative experiments with three biological replicates per treatment. Error is reported as standard deviation. C) A representative fluorogram after treatment with ML336. Cells were treated with ML336 at $2.5 \mu \mathrm{M}$ or 
DMSO at the time points indicated and then the RNA was visualized by treatment with a scintillant and exposure to X-ray film as described in the materials and methods. $1 \mu \mathrm{g}$ of RNA was used per lane. G: genomic RNA, SG: subgenomic RNA. 


\section{ML336 inhibits VEEV RNA synthesis in a cell-free system.}

The work outlined here implies that ML336 and related compounds function to inhibit VEEV infection by interfering with the synthesis of viral RNA. And this activity is specific to viral RNA and has minimal effect on cellular RNA synthesis (Figure 10). Further, it is known that the replication of viral RNA is carried out directly by the virally derived replicase complex ${ }^{58-61}$. This catalytic activity is also maintained by purified protein ${ }^{124,125}$ Additionally it has been previously found that viral isolates that resist treatment with these antiviral compounds have mutations in the $\mathrm{N}$ terminal region of nsP2 and nsP4 ${ }^{148}$. When taken altogether, this led to our hypothesis that ML336 and related compound were likely to be directly interacting with the viral nsPs to mediate their anti-VEEV activities. Currently there has been no successful expression of the full length nsP2 protein of any alphaviruses, and the other nsPs are also known to be difficult to ectopically. This limited our abilities to examine a potential interaction between these proteins and ML336. This led to our use of a so-called cell-free RNA synthesis assay.

The cell-free RNA synthesis assay was developed for alphaviruses many years ago and uses fractioned cellular components as both enzyme and template to generate viral RNA in the absence of complete, living cells ${ }^{166170}$. While this method does not completely remove cellular components from the assay, it greatly limits the amount of cellular proteins present, and is currently the best that can be done to isolate and examine the activities of the nsPs directly. The use of the P15, or membranous fraction, provides both template and polymerase to examine viral RNA synthesis. P15 fraction isolated from VEEV-infected cells was incubated with ribonucleotide substrates (e.g., ATP, GTP, and UTP) and radioactive CTP in the presence of ML336 or DMSO, then the in vitro synthesized viral RNA was analyzed on denatured agarose gels. 
As shown in Figure $12 \mathrm{~A}$, while there was no radiolabeled RNA produced by the P15 fraction of uninfected cells, three distinct viral RNA bands corresponding to genomic (49S), subgenomic (26S), and a replication intermediate, which has been previously reported on denaturing gels ${ }^{166}$, were present in the RNA produced with P15 from virusinfected cells. The addition of ML336 in the reaction decreased the amount of all three RNA species in a dose-dependent manner. At ML336 concentrations greater than 200 $\mathrm{nM}$, there was complete abrogation of all viral RNA synthesis. This result, complete inhibition of viral RNA synthesis of the P15 fraction, indicated that the inhibition of viral RNA synthesis by ML336 is independent from cellular metabolism and supported our hypothesis that ML336 inhibits viral RNA synthesis by a direct interaction with the viral replicase complex.

The $I_{50}$ value in this assay was then determined via optical density analysis from three separate experiments, and was found to be $49 \mathrm{nM}$, which is similar to the $\mathrm{EC}_{50}$ value as determined in our initial cell-based assays, Figure $12 \mathrm{~B}$. This $\mathrm{IC}_{50}$ value is higher than that seen for RNA inhibition in our $3 \mathrm{HU}$ incorporation assay, Figure $9 \mathrm{~A}$. This is most likely due to differences in the RNA labeling reactions. The $3 \mathrm{HU}$ incorporation assay labels all of the viral RNA that is being produced in the cell. However the cell-free synthesis assay is labeling primarily positive-sense RNA manufactured by mature replicase complexes. This could lead to less sensitivity as ML336 also inhibits the synthesis of negative-sense RNA.

The autoradiogram in Figure $12 \mathrm{~A}$ and also shows a large amount of small RNA products being labeled that were not present in the fluorgram in Figure $11 \mathrm{C}$. This is due to the being different ways of making and labeling RNA. The fluorgram uses total isolated RNA from infected cells, which is made in an ideal system for the virus. The labeling periods are also rather long allowing for highly efficient incorporation of label 
into the RNA. The autoradiogram, by contrast, is in a limited system, with minimal resources for the replicase complex. This minimal system is probably the cause of the increase in labeled small transcripts. These transcripts could be early terminated RNA, RNA synthesis initiating incorrectly, or RNA in the middle of synthesis 
A

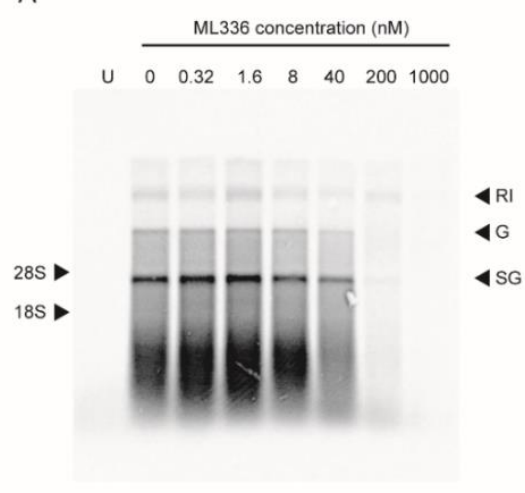

B

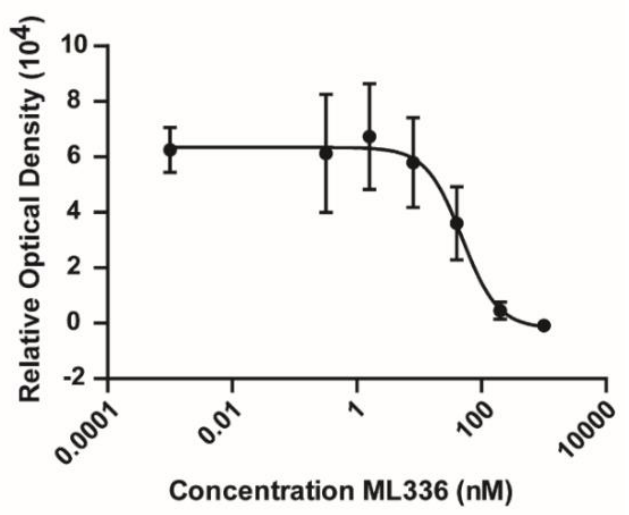

Figure 12. ML336 is active in a cell-free system.

The P15 was isolated from BHK 21 cells infected at an MOI of 10 with VEEV TC-83. This material was combined with various reagents as described in the methods, with [a33P]-CTP to label viral RNA. A) A representative RNA gel showing the results of the labeling reaction. The $28 \mathrm{~S}$ and $18 \mathrm{~S}$ ribosome subunit $\mathrm{RNA}$ were used as size markers. RI: a VEEV replication intermediate; G: the VEEV genomic RNA; SG: the VEEV subgenomic RNA. $U$ is an uninfected control. $0 \mu \mathrm{M}$ is a DMSO treated control. RNA amounts were controlled by using equal numbers of infected cells $\left(1.25 \times 10^{6}\right)$ per reaction, and total isolated RNA was used from each reaction. B) Densitometry was performed on three images from separate experiments to quantify the amount of labeled RNA present. These results are shown as a dose response curve. The $\mathrm{IC}_{50}$ value in this assay was found to be $49 \mathrm{nM}$. 


\section{Discussion}

ML336 and its analogues have been discovered through medicinal chemistry efforts originating from a quinazolinone hit compound that was identified from a cellbased high-throughput assay using live virus ${ }^{127}$. While our previous approaches using cell-based assays and mapping of resistance mutations strongly suggested viral RNA synthesis as the target step of this antiviral activity, the mechanism of action of the

compounds has remained unclear ${ }^{127,145}$. In this study we employed additional cell-based and biochemical assays to evaluate the effect of these compounds on VEEV viral RNA and host cell synthesis. Our data suggest that ML336 and related compounds inhibit VEEV by directly preventing viral RNA synthesis via a direct interaction with the viral replicase complex. ML336 demonstrated an efficacious inhibitory activity in both cellbased and cell-free viral RNA synthesis assays, and showed strong inhibitory activity against the synthesis of all viral RNA species. Our data strongly support the conclusion that the primary anti-VEEV mechanism of the amidine compound, ML336, is interfering with viral RNA synthesis.

The inhibition of viral RNA synthesis mediated by interaction with the replicase complex has not yet been described for any antiviral with efficacy against alphaviruses. With regards to the molecular mechanism of these compounds, resistant mutations in nsP2 (e.g., nsP2 $\mathrm{Y} 102 \mathrm{C}$ ) indicate that this region of nsP2 may be important for sensitivity to ML336. A homology model of VEEV nsP2 made using a recently published crystal structure of the $\mathrm{N}$ terminal region of nsP2 of CHIKV with the I-TASSER protein modeling server $^{171-173}$, Figure 14, has shown that the residues that are important for sensitivity to ML336 are located in an accessory domain to the helicase, termed the stalk domain, in nsP2 ${ }^{98}$. The stalk domain is a large alpha helix that is external to the active site of the 
helicase, which may imply that this region could be involved in the protein interactions in the replicase complex during infection. 


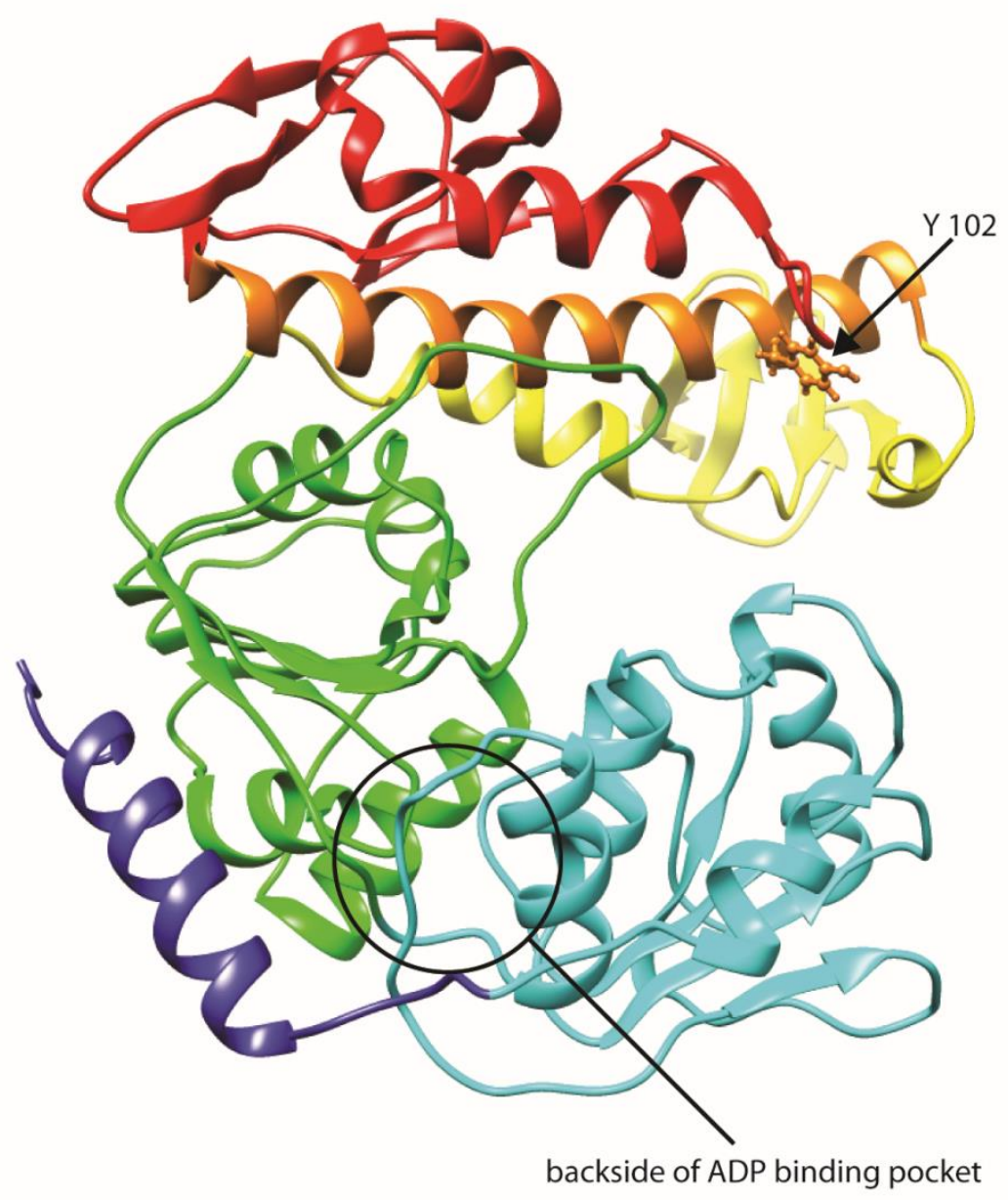

Figure 13. Homology model of VEEV nsP2 $\mathrm{N}$ terminal region.

A ribbon structure homology model of the first 465 amino acids of VEEV nsP2 was produced using I-TASSER. This model was made possible by the recent publication of the crystal structure of this same protein region from CHIKV by the Luo lab ${ }^{98}$ which was used as the basis for our model. Domains are color coded. $\mathrm{N}$ terminal domain (red), Stalk domain (orange), 1 B (yellow), Rec 1 (green), Rec 2 (light blue), Connector (dark 
blue). The region where our compounds are expected to bind is the stalk in the stalk domain. The location of one of the resistance residues, $Y_{102}$, is indicated with the side chain. The ADP binding pocket is also marked. 
Our work characterizing the benzamidine compounds also has the potential to lead to further understanding of the divergence between the New- and Old-World alphaviruses. The Old-World alphaviruses (e.g., CHIKV) do not show susceptibility to this scaffold as it relates to RNA synthesis inhibition. The $n s 2_{102}$ residue is occupied with a tyrosine in New-World alphaviruses (i.e., EEEV, VEEV, and WEEV); while a lysine residue occurs at this position in the Old-World alphaviruses. This information provides insight into novel ways in which the $\mathrm{N}$-terminal regions of $\mathrm{nsP} 2$ and nsP4 may affect the divergent phenotypes of the New- and Old-World viruses. Lastly, further research to understand the mechanism of ML336 may assist in the design of new compounds that inhibit replication of the Old-World alphaviruses.

In conclusion, we demonstrated that ML336 is a selective and potent inhibitor of VEEV viral RNA synthesis and that the inhibition of viral RNA synthesis might be the primary antiviral mechanism of this class of compounds. Our results strongly support that this class of compounds has a high potential for effective antivirals for New World alphaviruses.

\section{Conclusions}

The previously discovered antiviral compound ML336 was found to inhibit the replication of viral RNA by VEEV. This activity appears to be mediated through domains of currently unknown function in the $\mathrm{N}$ terminal regions of viral nonstructural proteins 2 and 4. Further characterization of this proposed interaction will be helpful in determining the function(s) of this domain which is currently proposed as an accessory domain to the helicase. We hypothesize that it will be involved in protein/protein interactions due to its location external to the helicase active site, as well as it showing no evidence of RNA binding activities. 
CHAPTER 3

ANALYSIS OF INTERACTIONS BETWEEN AMDIDINE COMPOUNDS AND THE VEEV NSPS 


\section{Overview}

As outlined in the previous chapter, ML336 and related compounds mediate their anti-VEEV activity by inhibiting viral RNA synthesis. Due to the specificity of this activity, as well as the fact that viral isolates that resist compound treatment have mutations in the $\mathrm{N}$ terminal regions of $\mathrm{nsP} 2$ and $\mathrm{nsP} 4^{127}$, we hypothesize that ML336 and related compounds interact with VEEV nsP2 and nsP4. To detect and characterize the hypothetical interaction(s) between the amidine compound family and Venezuelan equine encephalitis virus (VEEV) it is desirable to determine the location and dynamics of this interaction. However, working with VEEV proteins in biochemical assays is difficult due to difficulty expressing them recombinantly. This chapter outlines various methods that were tested in an attempt to characterize this interaction, as well as the development of a system for the ectopic expression of VEEV nsP2.

\section{Introduction}

While the mechanism of action of our amidine family anti-VEEV compounds has been thoroughly examined, the interaction(s) between these drugs and their targets has yet to be fully characterized. Characterizing these interactions will provide more detail about the mechanism of action, as well as assist in the further development of this compound scaffold. It was hypothesized that ML336 and related compounds interact with the nsPs of VEEV. This is due to several observations. First, when viral isolates occur that resist treatment with this compound family, the mutations that occur in these viruses are primarily focused in nsP2 and nsP $4^{127}$. This indicates that these regions and proteins are important in mediating compound activity. Second, ML336 acts to inhibit viral replication and CPE by interfering with viral RNA synthesis. This activity is known to be carried out by the viral nsPs. Lastly, ML336 remains activity in a cell-free RNA synthesis assay. While this assay does not eliminate all cellular proteins, it does 
eliminate all living cells. This indicates that the antiviral activity of this compounds is unlikely to be dependent on cellular signaling processes.

However, the detection of these hypothetical interactions between ML36 and the viral nsPs is difficult, as most methods of interaction detection require both large amounts of starting material, in this case of viral protein, as well as highly pure sample. There are often challenges in expressing eukaryotic, or in this case viral, proteins such as insolubility, disulfide bond formation, and post-translational modification ${ }^{174,175176,177,}$ and the VEEV nsPs have been historically difficult to generate in this manner, as can be seen in only partial expression of nsP2 that has been achieved ${ }^{98,102}$.

To date, there has been relatively little success in solving the structures of the VEEV nsPs, which makes the prediction of binding sites and interaction using in silico methods challenging. While the macrodomain of nsP3, and the protease of nsP2 have solved structures ${ }^{102,108,178}$, the remaining proteins and domains have proven difficult to work with and only recently has there been published work examining parts of their structure. The $\mathrm{N}$ terminal region of nsP2 has recently been crystalized from CHIKV, and this allowed for the development of homology models of this protein in VEEV98. A cryoem derived structure of nsP1 was also recently published in its membrane bound form ${ }^{92}$. The crystal structure of nsP2 has paired knowledge of protein structure with previous research that this protein contains several biologically active domains, in particular the C-terminus contains a cysteine like protease domains, while the $\mathrm{N}$ terminus has helicase and RNA binding activity ${ }^{88,96,162}$. The C-terminal region of nsP2 also contains a methyl transferase like domain, however this domain has not been confirmed to be functional ${ }^{85}$, however it appears to involved in innate immune signaling ${ }^{105}$. Mutations in this region also interfere with the localization of nsP2 to nucleus ${ }^{179}$

The determination of protein function and the production of proteins for various biochemical assays is greatly aided by the use of vector systems for the expression of 
proteins. The most common vector system is the use of specially designed strains of $E$. coli that are optimized for this purpose. These systems typically utilize inducible expression systems that can be controlled by media supplementation ${ }^{174}$. There are also many strains available that have been optimized for the expression of difficult eukaryotic proteins $^{174}$.

With the goal of examining the hypothetical interactions between ML336 and the VEEV nsPs, several methods of protein isolation and labeling were attempted. The methods that were utilized were fractioning of cells and ultracentrifugation, the labeling of proteins and tracking of interactions using Click-iT chemistry, and recombinant expression of VEEV nsP2.

It was first attempted to purify the nonstructural proteins from infected cells. Previous work has established that these proteins could be detected in highly purified spherules from infected cells ${ }^{74}$. So, we sought to enrich for this fraction from infected cells and then determine if we could use this fraction for biochemical binding assays. After finding little success with this approach, a recently developed chemical labeling system referred to as Click-iT chemistry ${ }^{180}$ was utilized.

This chemistry utilizes functional groups that are essentially absent in biological systems, azides and alkynes, to perform highly specific interactions which form covalent linkages between these groups. These interactions can be used to label molecules for imaging, for isolation of molecules form complex mixtures, and many other downstream applications. Multiple types of reactions have been optimized both with and without copper catalysis. In this instance proteins are metabolically labeled with a methionine analogue that can be then be appended using a marker than can then be purified or tracked with imaging techniques ${ }^{181182183}$. This system is advantageous as it can be performed during infection easily, and depending on the method used requires minimal protein for detection. Working with our medicinal chemist collaborator, we also obtained 
two compounds, one with efficient antiviral activity and one without, that were modified to contain click labile functional groups. This allows for these compounds to be directly labeled in the Click reaction and detected in a variety of ways.

While this system showed promise, being able to detect both metabolically labeled proteins as well as specifically appended click labile antiviral compounds, these methods were not specific enough for our needs. We detected primarily cellular proteins after metabolic labeling, and the click labile compounds were too dispersed throughout the cells to make colocalization with viral replication centers or proteins possible.

Lastly, an expression system was developed for nsP2 and nsP4. The work of expressing and purifying this protein was performed iteratively, starting from attempting to express fusion peptides of nsP2 and nsP4 in multiple orientations. It was hypothesized that these proteins interact in the replicase complex due to resistance mutations occurring in both of these proteins in response to treatment with the small molecule antiviral compounds. This initial strategy eventually led to the successful expression of the $\mathrm{N}$ terminal region of nsP2 using a large solubility increasing tag in a highly specialized strain of $E$. coli termed Rosetta, that has been altered for the expression of both proteins with a large number of disulfide bonds as well as for codons that are rare in $E$. coli $i^{174}$.

\section{Materials and Methods}

\section{Cell culture and viral strains}

Baby hamster kidney (BHK) clone 21 cells (ATCC CCL-10) and Vero 76 (African Green monkey kidney fibroblasts) (ATCC® $\mathrm{CRL}-1587^{\mathrm{TM}}$ ) were maintained in Modified Eagle's Medium with Earle's Balanced Salt Solution and L-glutamine (MEM-E, Corning 10-010-CV) supplemented with 10\% fetal bovine serum (FBS) (Corning 35-011-CV). Cells were maintained at $37^{\circ} \mathrm{C}$ in humidified incubators with $5 \% \mathrm{CO}_{2}$. VEEV strain TC-83 
(gift of Dr. Connie Schmaljohn, USAMRIID) was used for this study. The strain V3526 was generated from a plasmid as described previously (Chung et al., 2014). Infections were carried out using a virus infection medium (Modified Eagle's Medium with Earle's Balanced Salt Solution with L-glutamine (Corning 10-010-CV), 25 mM HEPES, 10\% FBS)

\section{Solubilization of the P15 fraction}

Cells were infected with VEEV TC-83 at $10 \mathrm{MOI}$ and incubated for 6 hours. Then, cells were washed with ice-cold, sterile PBS (Caisson Labs PBL01) and the cells were incubated in hypotonic $\mathrm{RS}$ buffer (10 mM NaCl, $10 \mathrm{mM}$ Tris-HCl, pH7.8) supplemented with Protease inhibitor cocktail III (Research Products International P50700-1) on ice for 15 minutes. Cells were scraped into buffer and thoroughly homogenized using a Dounce homogenizer. Nuclei were removed by centrifugation at $900 \mathrm{xg}$ for 10 minutes at $4^{\circ} \mathrm{C}$. Supernatant containing the cytoplasmic fraction was transferred to microcentrifuge tubes and centrifuged at $15,000 \times \mathrm{g}$ for 20 minutes at $4{ }^{\circ} \mathrm{C}$. The supernatant ( $\mathrm{S} 15$ fraction) was removed and pellets (P15 fraction) were used for further analysis.

To solubilize the membranes and release the viral proteins the $\mathrm{P} 15$ fraction was treated with either Triton X-100 (Sigma Aldrich T-9284) (2\% v/v) or sodium deoxycholate (Sigma Aldrich 30970$)(\mathrm{DOC})(0.5 \% \mathrm{w} / \mathrm{v})$ with or without $1 \mathrm{M} \mathrm{NaCl}$ at $4^{\circ} \mathrm{C}$ for ten minutes with mixing at $750 \mathrm{RPM}$. The insoluble material was then removed by centrifugation at $15 \mathrm{k} \times \mathrm{G}$ for 20 minutes at $4 \mathrm{C}$. The supernatants were then used in a cell free RNA.

Method adapted from Pietla et $\mathrm{al}^{74}$

\section{Isolation of viral proteins via glycerol and sucrose gradients}

Supernatants from DOC solubilized P15 fractions were used as the input material for ultracentrifugation over a glycerol gradient. A discontinuous gradient was made using three steps 15\%, 23\%, and 30\% glycerol with $1 \mathrm{mM}$ EDTA (Promega V4231) , $50 \mathrm{mM}$ 
Tris- $\mathrm{HCl} \mathrm{pH}=7.8,0.5 \% \mathrm{DOC}(\mathrm{w} / \mathrm{v})$ and $150 \mathrm{mM} \mathrm{NaCl}$. Samples were centrifuged at 100,000 RPM for 1 hour in a Beckmann TLA 110 rotor at $4^{\circ} \mathrm{C}$. Ten fractions of $500 \mu \mathrm{L}$ were collected by bottom puncture and those with the highest activity as measured by RNA synthesis assay were further separated via a sucrose gradient.

A three step discontinuous sucrose gradient was used to further separate the fractions that were found to have RNA synthesis activity after the initial separation on the glycerol gradient. The steps were $15 \%, 30 \%$, and $60 \%$ sucrose $(\mathrm{w} / \mathrm{v})$. The samples were centrifuged at 100,000 RPM for 6.5 hours in a TLA 110 rotor at $4^{\circ} \mathrm{C}$. Ten fractions of 500 $\mu \mathrm{L}$ were collected by bottom puncture. Fractions were assayed for activity in the RNA synthesis assay.

\section{Isolation of viral proteins using cesium chloride}

Cesium chloride is used to create continuous gradients during centrifugation, and separates cellular components based on density rather than migration speed as opposed to glycerol and sucrose gradients. An aqueous solution of $1.37 \mathrm{~g} / \mathrm{mL} \mathrm{CsCl}$ was made and loaded into centrifuge tubes. The P15 from infected cells was isolated and solubilized as described above. The samples were loaded on top of the gradient and centrifuged at $100,000 \times \mathrm{G}$ for eight hours at $4^{\circ} \mathrm{C}$ in a Beckmann TLA 110 rotor. Ten fractions of $500 \mu \mathrm{L}$ each were collected by bottom puncture. Floating material that did not enter the gradient was also collected.

\section{Immunoblotting}

The collected fractions after glycerol centrifugation were combined with Laemmli buffer (4\% SDS w/v, 20\% glycerol w/v, $120 \mathrm{mM}$ Tris-HCl pH=6.8, bromophenol blue) 2:1 and boiled to denature proteins. The prepared samples were loaded into gradient gels for SDS-PAGE (GenScript M00656) and run at 150V until the dye front reached the bottom of the gel. Protein was transferred to a PVDF membrane (BioRad 1620177) using methanol Tris-glycine buffer ( $200 \mathrm{mM}$ glycine, $25 \mathrm{mM}$ Tris, $10 \%$ methanol v/v) for 
two hours at $20 \mathrm{~V}$. Membranes were washed once with $1 \mathrm{X}$ TBS and blocked with $5 \%$ dry milk in TBS at room temperature for one hour. The membrane was washed five times in TBS- $0.01 \%$ tween 20 , and then stained with primary antibody overnight at $4^{\circ} \mathrm{C}$. The nsP2 monoclonal antibody was used at a final concentration of $0.4 \mu \mathrm{g} / \mathrm{mL}$ diluted in TBST. The membrane was then washed five times with TBS-T and stained with secondary antibody diluted in TBS-T. Anti-mouse HRP stained the nsP2 antibody and is used at a final concentration of $1: 10,000$. The secondary antibody staining was performed for one hour at room temperature. The membranes are washed six times in TBS-T and developed in ECL reagent for five minutes at room temperature. The images were collected using an Azure Biosystems c300 imaging system for detection of chemiluminescence.

\section{Cell-free RNA synthesis assays}

VEEV viral RNA synthesis assay was adapted from Barton et al ${ }^{166}$. Ten microliters of P15 fraction enriched for VEEV viral replicase complexes, which is equivalent of approximately $1.25 \times 10^{6}$ infected cells, was combined with a same volume of a RNA synthesis mix (100 mM Tris- $\mathrm{HCl} \mathrm{pH} 7.8,100 \mathrm{mM} \mathrm{KCl}, 20 \mu \mathrm{g} / \mathrm{mL}$ act D, $20 \mathrm{mM}$ DTT, $10 \mathrm{mM}$ creatine phosphate (Sigma Aldrich 10621714001) , $50 \mu \mathrm{g} /$ reaction creatine phosphokinase (Sigma Aldrich C3755-3.5KU), 4 mM of ATP, GTP, and UTP, $20 \mu \mathrm{M}$ CTP (NEB N0450s), $12 \mathrm{mM} \mathrm{MgCl}_{2}$ ) on ice and $1 \mu \mathrm{L}$ of SUPERaseln RNase inhibitor (Ambion AM2694 ), $5 \mu \mathrm{g}$ of yeast tRNA (Ambion 15401011), and $5 \mu \mathrm{Ci}$ of [ $\left.\alpha{ }^{-33} \mathrm{P}\right]-\mathrm{CTP}$ (Perkin-Elmer NEG608H) were added per reaction. After an incubation at $37^{\circ} \mathrm{C}$ for 90 minutes, RNA was isolated from each reaction using RNAzol RT and RNA mini prep kit columns (Zymo Research R2052) according to manufacturer's instructions, with an additional wash step before elution. For compound addition, ML336 was added to reaction mixtures before incubation at $37^{\circ} \mathrm{C}$ at the indicated concentrations. The final DMSO (Sigma Aldrich D8418) concentration was 0.25\%. 


\section{Silver stain of total protein}

The fractions collected after $\mathrm{CsCl}$ centrifugation were mixed 2:1 with Laemmli buffer (4\% SDS (RPI L22010) w/v, 20\% glycerol w/v, 120 mM Tris-HCl pH=6.8, bromophenol blue) and boiled to denature the proteins. The prepared samples were analyzed by SDS-PAGE in a gradient gel, run at $150 \mathrm{~V}$ until the dye front reached the bottom of the gel. Gel was stained using the Pierce Silver Stain Kit (Thermo Fisher 24612) according to manufacturer's directions. Gel was digitally scanned.

\section{L-AHA labeling of proteins}

Cells were infected with VEEV TC-83 at an MOI of ten and held on ice for one hour. The cells were washed once with PBS and released into warm infection media. Infection proceeded for 8 hours. The infected cells were then starved of methionine 30 minutes before labeling was begun by incubation in DMEM hi-glucose without methionine, cysteine, or sodium pyruvate (ThermoFisher 21013024). Cells were then treated for one hour with azidohomoalanine (Anaspec AS-63669) (L-AHA) a methionine analog which contains an azide, at a concentration of $25 \mu \mathrm{M}$.

\section{DIBO labeling of L-AHA treated cells}

Dibenzocyclooctyne (DIBO) is the catalyst for copper free click chemistry. This version of click chemistry is useful because it avoids the off target effects of copper in cells ${ }^{184}$. For labeling of live cells, the cells are washed twice with PBS then DIBOAlexafluor 647 (ThermoFisher C20022) in PBS was added to the wells to a final concentration of $5 \mu \mathrm{M}$. The cells were rocked in the dark at room temperature for one hour. The cells were washed twice with PBS then lysed with lysis buffer (1\%SDS (w/v), $50 \mathrm{mM}$ Tris- $\mathrm{HCl} \mathrm{pH}=8$ ). Cells were held in lysis buffer for fifteen minutes on ice, suspended and moved to Eppendorf tubes, then sonicated for five minutes in a water bath sonicator. The lysate was centrifuged for five minutes at max speed at $4 \mathrm{C}$ to 
remove any debris. For DIBO labeling of cell lysate, DIBO-Alexafluor 647 was added to a final concentration of $5 \mu \mathrm{M}$ to the cell lysate. Lysate was incubated in the dark for one hour at room temperature.

\section{Copper containing Click-iT reaction}

Cells were infected at an MOI of 10 with VEEV TC-83. Cells were held on ice for one hour. Cells were washed once with PBS and then released into warm infection media. Infection proceeded for 3.5 hours and the cells were methionine starved as before. The cells were treated with L-AHA at $25 \mu \mathrm{M}$ for four hours. The cells were lysed as before, and the proteins were precipitated using methanol:chloroform precipitation. The proteins were then labeled using the Thermo Click-iT (C10276) protein reaction buffer kit according to manufacturer's instructions. The labeled proteins were separated by SDS-PAGE, 150V until the dye front reached the bottom of the gel, and images collected using a BioRad Pharos imaging system.

\section{Treatment of infected cells for fluorescent microscopy}

BHK-21 cells grown on glass coverslips were infected at an $\mathrm{MOI}$ of 0.25 and held on ice for 1 hour to synchronize infection. Cells were washed with PBS and warm infection media was added to initiate infection. Infection proceeded for 5.5 hours at $37 \mathrm{C}$ and $5 \% \mathrm{CO} 2$. The media was aspirated, and the cells were pulsed with media containing either of the two compounds at $10 \mu \mathrm{M}$ or $0.25 \%$ DMSO vehicle control. Compounds 3260 and 3261 are derived from the amidine scaffold backbone, they differ in that they each contain both an azide and alkyne functional group. The azide is photolabile and used to UV fix the compounds to whatever they are bound to in the cell. The alkyne is then used from Click chemistry. Cells were incubated for 30 minutes at $37^{\circ} \mathrm{C} 5 \% \mathrm{CO}_{2}$. The cells were washed for ten minutes in HBSS (Lonza 04-315Q). The cells were short wavelength UV fixed for ten minutes to immobilize the compounds. The cells were fixed in a one-to-one mix of acetone and methanol at $-20^{\circ} \mathrm{C}$ for 20 minutes in preparation for 
an immunofluorescence assay (IFA) and to permeabilize the cells. The click reaction was then carried out on the monolayer: $1 \mathrm{mM} \mathrm{CuSO}_{4}, 100 \mathrm{mM} \mathrm{L-ascorbic} \mathrm{acid} \mathrm{sodium}$ salt, $20 \mathrm{mM}$ Tris- $\mathrm{HCl} \mathrm{pH}=8.5,20 \mu \mathrm{M}$ A647 alkyne. $300 \mu \mathrm{L}$ of the solution was added per well, and the plates were incubated in the dark at room temperature for 30 minutes. Cells were washed four times with PBS and then used for antibody staining.

\section{Staining of cells for microscopy}

After the click reaction to directly detect the click labile antiviral compounds, the cells were stained to detect VEEV nsP2 and double stranded RNA. The antibodies were diluted into PBS with $0.1 \%$ saponin (Sigma Aldrich 47036) with $1 \%$ normal horse serum. Anti-VEEV E protein (BEI) was used at a concentration 1:10,000, and JS1 anti-dsRNA antibody (obtained from the lab of Dr. Nobuyuki Matoba as a human FC switched antibody) at a concentration of 1:1000. Primary antibody staining was carried out overnight at $4^{\circ} \mathrm{C}$. The cells were washed three times with PBS-saponin and then stained with secondary antibody in the same buffer as before. Anti-goat 555 (gift from the AbuKwaik lab) was used at a concentration of 1:1,000 to stain for $E$ protein while anti human 488 (JIR 709-545-149) was used to stain for dsRNA at a concentration of 1:1,000. Secondary staining was carried out at room temperature for one hour. The cells were washed four times with PBS saponin. The cells were incubated with Hoechst stain diluted 1:10,000 in PBS for ten minutes at room temperature. Slips were removed from the plate and mounted on glass slides using Prolong Gold Antifade mounting medium with DAPI (Invitrogen P36931).

\section{Design of protein expression plasmids}

Initially we deigned pET19.b plasmids that expressed fusions of the $\mathrm{N}$ terminal domains of nsP2 and nsP4 in two orientations, nsP2-nsP4 and nsP4-nsP2 with a flexible linker between the two regions, a map of one such insertion is shown in Figure 17 
(custom expression plasmids from GenScript). These constructs were insoluble. We next attempted to express the $\mathrm{N}$ terminal domain of $\mathrm{nsP} 2$ as carried out by the Luo lab ${ }^{98}$. Expression of the $\mathrm{N}$ terminal domain of nsP2 was performed using the same Pet19.b plasmid backbone. The protein construct encoded a his tagged SUMO solubility tag, immediately followed by the first 465 amino acids of VEEV nsP2. This construct and plasmid backbone is found in Figure 16 (custom expression plasmids from GenScript).

\section{Strains of bacteria used for expression}

All protein expression experiments were carried out using E. coli. All strains are based on the BL21 (DE3) strain of E. coli which contains the genes necessary for inducible expression of protein using isopropyl-B-d1-

thiogalactopyranoside (IPTG). Strain BL21 (DE3) was used for expression of the expression of the nsP2-4 fusion peptides. E. coli Rosetta (Novagen 70594, gift from the lab of Dr. Donghan Lee) was used for expression of the N-terminal region of nsP2.

\section{Expression of the $\mathbf{N}$ terminal domain of VEEV nsP2}

The expression vector was transformed into E. coli Rosetta, and then used for protein expression. Cultures were grown at $37^{\circ} \mathrm{C}$ in LB broth to an OD600 of 0.4 and induced with the addition of $1 \mathrm{mM} \mathrm{IPTG}$. The cultures were shifted to $18^{\circ} \mathrm{C}$ and then grown overnight.

\section{Isolation of bacterially expressed proteins}

The bacterial cultures were centrifuged to pellet. Three $\mathrm{mL}$ of cell lysis buffer was added per gram of $E$. coli cell pellet (50 mM Tris- $\mathrm{HCl} \mathrm{pH}=8,1 \mathrm{mM}$ EDTA (Promgea V4231) pH=8, $500 \mathrm{mM} \mathrm{NaCl}, 1$ mM DTT (Enzo ALX-280-001G025))) and the bacteria were resuspended. Four microliters of $100 \mathrm{mM}$ 
phenylmethylsulphonyl fluoride (PMSF), and $80 \mu \mathrm{L}$ of ten $\mathrm{mg} / \mathrm{ml}$ lysozyme were added per gram of $E$. coli. This mixture was agitated for 20 minutes at $4^{\circ} \mathrm{C}$. Four milligrams of sodium deoxycholate were added per gram of E. coli and this mixture sonicated on ice to ensure complete lysis. The lysate was centrifuged at 5000 RPM for 1 hour at $4^{\circ} \mathrm{C}$ in a Beckman JS-5.3 rotor. The supernatant is the soluble fraction.

\section{Recovery of protein from inclusion bodies to determine localization during expression}

To recover protein from the inclusion bodies, the pellet was resuspended in $30 \mathrm{~mL}$ of cell lysis buffer $2(50 \mathrm{mM}$ Tris $-\mathrm{HCl} \mathrm{pH}=8,10 \mathrm{mM}$ EDTA $\mathrm{pH}=8,500$ $\mathrm{mM} \mathrm{NaCl}, 0.5 \% \mathrm{v} / \mathrm{v}$ Triton $\mathrm{X}-100)$, and incubated for five minutes at room temperature in a water bath. The solution was centrifuged at 5000 RPM for twenty minutes at $4^{\circ} \mathrm{C}$ and the supernatant discarded. This was repeated twice, for a total of three washes. The pellet was then washed with $30 \mathrm{~mL}$ of water and centrifuged as before.

The supernatant was discarded, and the pellet was suspended in an equal volume of inclusion body solubilization buffer $(50 \mathrm{mM}$ Tris- $\mathrm{HCl} \mathrm{pH}=8,1 \mathrm{mM}$ EDTA $\mathrm{pH}=8,500 \mathrm{mM} \mathrm{NaCl}, 8 \mathrm{M}$ urea, $0.1 \mathrm{mM} \mathrm{PMSF}$ freshly added) as the volume of the soluble fraction collected. This mixture was stirred at room temperature for one hour. Any remaining insoluble material was removed by centrifugation at 5000 RPM for twenty minutes at $4^{\circ} \mathrm{C}$. This is the purified inclusion body fraction. Purification of recombinantly expressed protein 
The expressed proteins contained histidine tags. To purify the desired protein nickel column chromatography was used (HisPure Ni-NTA resin Thermo Fisher, 88222). Resin was packed into a gravity column and washed and prepared according to manufacturer's instructions. Protein samples were equilibrated in PBS with $10 \mathrm{mM}$ imidazole. Increasing amounts of imidazole in PBS were used to elute the protein as indicated, ranging from 30 to $310 \mathrm{mM}$. Each elution was three resin bed volumes ( $3 \mathrm{~mL}$ of imidazole solution for $1 \mathrm{~mL}$ of packed resin). All purification steps were performed at $4^{\circ} \mathrm{C}$

\section{SDS-PAGE analysis of expressed protein}

Protein load was controlled based on OD600 of the bacterial sample. Sample was suspended in $100 \mu \mathrm{L}$ buffer per OD unit. $20 \mu \mathrm{L}$ of sample was combined with Laemmli buffer and separated in gradient gels. Gels were run at 150V until the dye front reached the bottom of the gel. Gels were stained with colloidal Coomassie (BioRad 1610803)

\section{Results}

Use of purified spherules for detecting the interaction between ML336 and related compounds with the VEEV nonstructural proteins

It has been previously shown that purified spherules from alphavirus infected cells contain RNA synthesis activity and contain nsPs ${ }^{74}$. It was determined that the protein contents of these spherules could be purified by centrifugation, and their protein contents could be utilized in assays to determine the interaction of the amidine scaffold compounds and the nsPs. 
The spherules can be isolated from infected cells by ultracentrifugation. Several gradient materials can be used for this purpose. In the case of these experiments, glycerol, sucrose, and cesium chloride were used. The proteins that were isolated were assayed for activity in the cell free RNA synthesis assay to confirm that their structure remained intact. While there was some activity maintained by the isolated protein after the initial glycerol separation, this activity was very weak compared to the starting material and was completely lost upon further isolation using a sucrose gradient. This indicates that structure of the protein complex was disrupted, and they were not suitable for further use, Figure $14 \mathrm{~A}$ and $\mathrm{B}$. This is likely due to disruption of the proteins during the centrifugation process. In previously published work the spherules were kept intact during centrifugation ${ }^{74}$. The disruption of these lipid layers is likely to leave the proteins more exposed to damage during the isolation process. Additionally a different media was used to form the gradient, a specialized sugar called iodixanol ${ }^{74}$. This specialized media has been used previously for the isolation of live cells ${ }^{185}$, and its particular properties probably assisted to keep the proteins protected.

There were also issues with the ultracentrifugation being able to isolate the nsPs to a single fraction, as shown in Figure $14 \mathrm{C}$, where nsP2 was detected in multiple fractions after ultracentrifugation, indicating that separation across the gradient was poor. This poor separation led to our hypothesis that separation by velocity, as in glycerol or sucrose gradients, may not be able to separate the cell components sufficiently in this case. These results led to our use of a $\mathrm{CsCl}$ gradient. This method creates a homogenous solution that then creates a density gradient during the centrifugation process which separates components based on their densities.

After centrifugation with $\mathrm{CsCl}$ most protein in the cellular extract remained floating on top of the solution and did not enter the column, Figure $14 \mathrm{D}$. This indicates that the proteins were contained in structures that greatly reduced their density. This 
most likely indicates that despite the initial solubilization to disrupt the lipids contained in the extract, enough of them remained intact to interfere with the separation of the proteins. After this was unsuccessful, other methods were examined that would allow for marking the proteins of interest in the infected cell instead of needing to extract them. This led to the use of click chemistry. 
A.

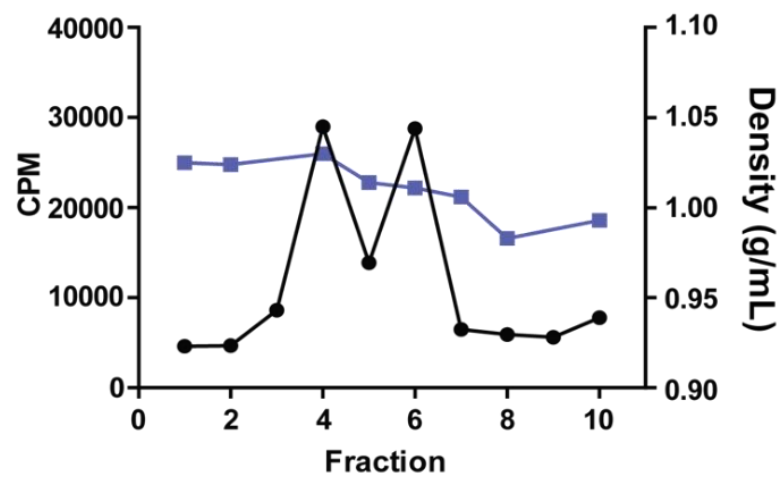

B.

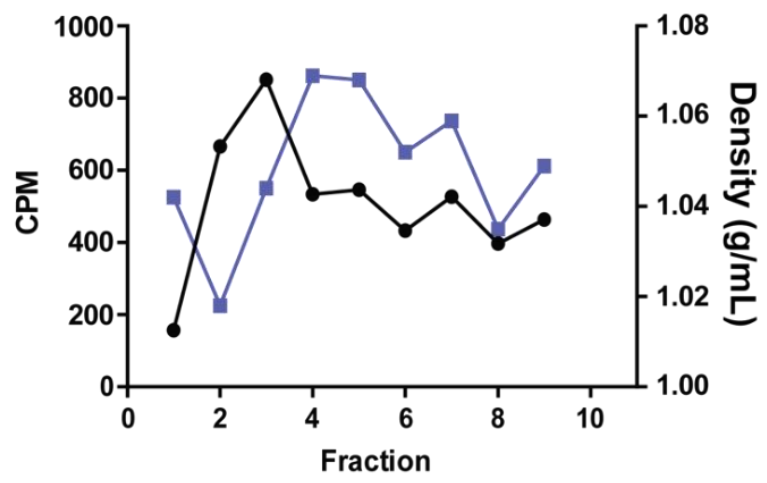

C. increasing glycerol \%

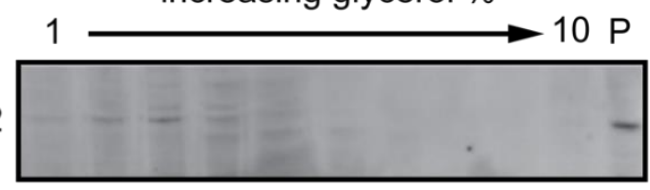

D.
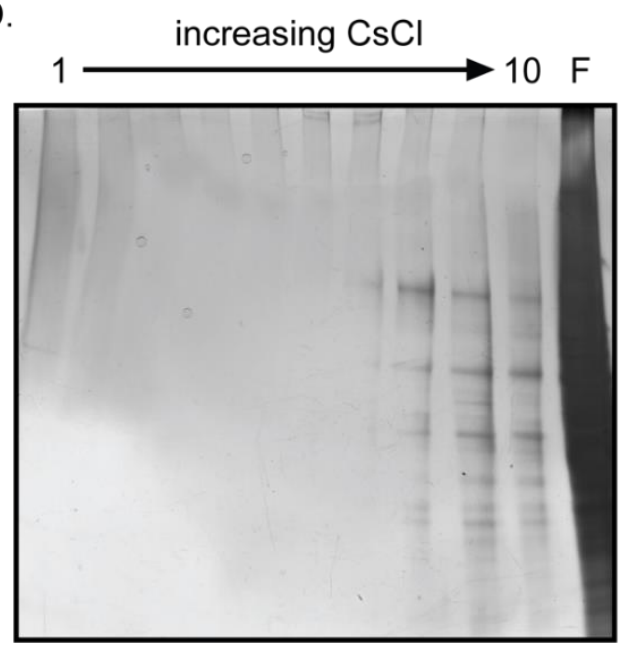

Figure 14. Isolation of the nsPs from infected cells using ultracentrifugation. A) P15 from infected cells was solubilized and separated over a stepwise glycerol gradient. After fraction collection the RNA synthesis activity, as measured by CPM using 33P 
incorporation, was isolated in fractions four and six. B) The active fractions were then further separated over a three-step sucrose gradient. After this further separation the RNA synthetic activity of these fractions was lost, image from one representative fraction. Graphs are from one representative experiment of three C) The nsPs did not cleanly separate across a glycerol gradient. After separation across a three step glycerol gradient, an immunoblot was performed to detect nsP2. NsP2 was detected in several fractions, and in a pellet, $\mathrm{P}$, that occur at the bottom of the centrifuge tube. Ten fractions were collected, increasing glycerol concentration from fraction 1 to 10 . Image is one representative experiment of 2. D) A continuous density gradient made using $\mathrm{CsCl}$ was also unable to separate the proteins. A solution of $\mathrm{CsCl}$ was generated and the solubilized P15 fraction loaded on top. After centrifugation ten fractions were collected, increasing density from fraction 1 to 10 . Fractions collected by bottom puncture. There was also a large amount of floating material $F$, that this did not enter the gradient and held a large majority of the proteins. The proteins were also present in a third of fractions at roughly equal level, indicating that separation was poor. Total protein was detected by silver staining. Isolation with $\mathrm{CsCl}$ was only attempted once. 


\section{The use of click chemistry to label proteins.}

Initially, the labeled proteins were extracted using methanol precipitation and then appended with fluorophore using the click reaction. Cells were infected at an $\mathrm{MOI}$ of ten with VEEV TC-83 for 3.5 hours. The cells were methionine starved for 30 minutes and then labeled with L-AHA for 4 hours. Cells were lysed and the proteins isolated using methanol precipitation. These proteins were then analyzed by gel electrophoresis, Figure $15 \mathrm{~A}$. However, there was poor detection of viral proteins generally, and the nsPs could not be resolved, indicating that this method was not sensitive enough to detect the proteins of interest specifically compared to the cellular proteins. It is important to point out that the specificity of this assay was based on the result of VEEV infection inhibiting the synthesis of cellular proteins. However, as can be seen, there was still extensive labeling of cellular proteins at the time point examined. Future experiments would need to either utilize later time points in infection, or use pharmacological inhibitors of cellular protein synthesis.

As metabolic labeling and in vitro tagging of the viral proteins was poorly sensitive and significantly labeled cellular proteins in addition to viral proteins, it was determined that altered antiviral compounds that already incorporated one of the functional groups would instead be used. Compound 3260 is an effective antiviral compound with an $\mathrm{EC}_{50}$ of $1 \mu \mathrm{M}$ in the CPE prevention assay, compound 3261 is an ineffective compound with and $\mathrm{EC}_{50}$ of greater than $50 \mu \mathrm{M}$. These compounds are derived from the same amidine backbone as ML336. Each contains both an azide and an alkyne moiety. The azide is photolabile and allows for the use of UV to fix the compounds in place in the cell. This degrades the azide, and then the alkyne is usable for click it chemistry. The advantage of this method is that these compounds are not subject to the changing efficiencies of metabolic labeling, and the potential association of 
the compounds and the viral proteins can be observed by co-staining with antibodies that detect the viral proteins. Two compounds were used, 3260 , which is an effective antiviral and was hypothesized to associate with the viral proteins, and 3261 which is not an effective antiviral and was predicted to not associate with the viral proteins.

Cells were infected with VEEV, and then treated with the compounds for 30 minutes. The cells were then fixed and permeabilized, and the click reaction was performed directly on the cell monolayers. The cells were then stained with antibodies against $E$ protein and dsRNA to determine the localization of replication and infection compared to the antiviral compounds.

As can be seen in Figure $15 \mathrm{~B}$, there was no significant association between either of the compounds tested and dsRNA staining the pink staining, indicating the labeled compounds, is distributed throughout all of the cells equally, regardless of infections indicated by the yellow $E$ protein or green dsRNA. The pink staining does not associated with either of those strains significantly within infected cells. While the active antiviral compound is expected to reduce dsRNA detection, due to the short treatment time (30 minutes) we had hoped that the compounds would not have taken full effect and that some viral replication would have remained active. Ultimately the only difference between the treatments was that the effective anti-VEEV compound, CB10003260, was able to enter cells at much higher levels compared to the ineffective anti-VEEV compound, CB10003261, used as a negative control. Importantly, this assay confirmed the antiviral effect of compound 3260 , as the cells treated with this compound had very little dsRNA staining, indicating an inhibition of viral RNA synthesis. And compound 3261 did not display this effect. 
A.

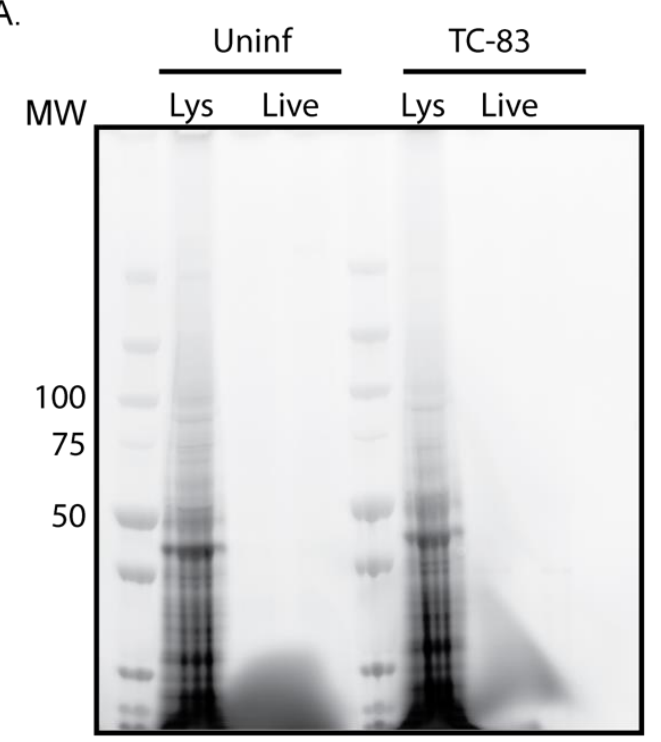

B.

E protein

dsRNA
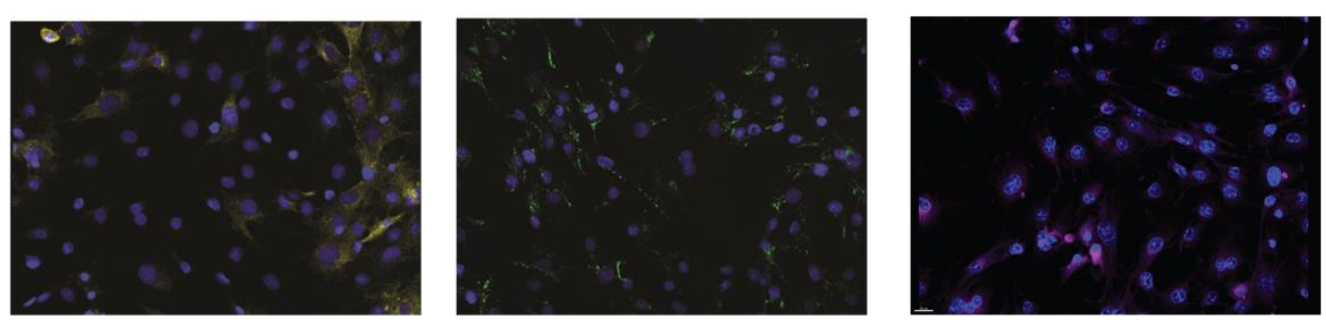

3260, E, dsRNA

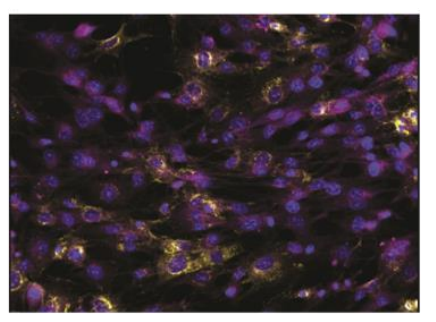

3261, E, dsRNA

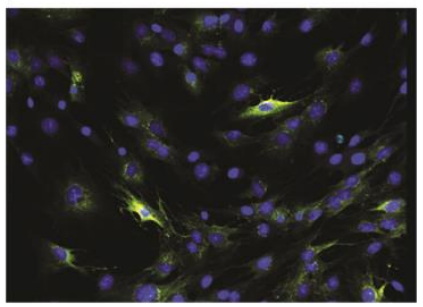

dsRNA

E protein

compound

nuclei

Figure 15. Use of click-iT to identify viral protein/compound interactions. A) Uninfected and TC-83 infected cells were metabolically labeled with L-AHA to mark all newly synthesized proteins for click it detection. Cells were infected at an MOI of ten for 3.5 hours and treated with L-AHA for 4 hours before lysis. The click it reaction was 
performed with DIBO A647 either in the live cells or on cell lysate. Cells were lysed and proteins precipitated. Proteins were run in a gel and then imaged with a BioRad pharos imager. Only the lysate showed significant click labeling. There was minimal detection of viral proteins compared to cellular proteins. One representative image of two experiments. B) Representative images of infected cells treated with click moiety compounds. Cells were infected at an $\mathrm{MOI}$ of 0.25 for 5.5 hours and treated with the indicated compounds for 30 minutes at $10 \mu \mathrm{M}$. Compound 3260 is active against VEEV and enters cells. Compounds 3261 is inactive and does not. There was no significant association between the compounds and either viral E protein or dsRNA. Representative images of one of two experiments. 


\section{Expression of recombinant protein for biochemical analysis}

The data from the click chemistry experiments was unable to confirm the hypothesis that the amidine antiviral compounds are associating with the nSPS of VEEV to mediate their activity. Having gone through several methods attempting to use proteins produced during infection, it was determined that we would attempt to recombinantly express and purify the proteins of interest. As mentioned above, E. coli was selected due to its ease of use and readily available reagents and genetic systems.

While it was known that expressing nsP2 in particular was likely to be difficult we started from an atypical approach of expressing the $\mathrm{N}$ terminal regions of nsP2 and nsP4 in fusion with one another. This fusion peptide was designed working under the hypothesis that these two domains interact closely in the mature replicase complex. This was hypothesized because of the high occurrence of compound resistant mutations in these two regions. Assuming that the antiviral compound binds to a single location in/on the replicase complex, for both of these regions to bind these small molecules they would need to be in close association with one another. We thus hypothesized that these regions are in close association, and that maintain this association in our protein constructs would result in enhanced folding and solubility during bacterial expression.

Two fusions were created, nsP2-4 and nsP4-2. The expression of these proteins was tested in E. coli BL21 (DE3). Maps of the protein constructs discussed here and the plasmid backbone used are shown in Figure 16. Neither of these two fusion proteins were able to be produced in a soluble manner, with both of them localizing to the inclusion body fraction of the $E$. coli after purification. Figure $17 \mathrm{~A}$ shows data from the nsP2-nsP4 fusion peptide. Various optimizations were carried out, e.g. altering the temperature of induction and the amount of inducer added to the media, but these made no difference in the solubility. Refolding was attempted but this was also unsuccessful. 
While the next steps were being determined, Luo group published the crystallization of the $\mathrm{N}$ terminal domain of nsP2 from $\mathrm{CHIKV}$, outlining the expression of the $\mathrm{N}$ terminal domain of nsP2 in a soluble manner by using $E$. coli Rosetta and a solubility increasing tag termed SUMO ${ }^{186}$. With their assistance, a new expression construct was designed that incorporated their methods for expression of the $\mathrm{N}$ terminal domain of VEEV nsP2. E. coli Rosetta was generously shared by the lab of Dr. Donghan Lee, as was a plasmid for the expression of the SUMO protease for removal of the tag during purification. As can be seen in Figure $17 \mathrm{~B}$, this new construct was indeed soluble when expressed in this strain of $E$. coli. And in Figure $17 \mathrm{C}$ it is shown that it is also readily purified using a Ni column for his tag purification.

Large amounts of this protein were synthesized, and then submitted to HPLC for final purification before use in binding assays. Unfortunately, after the HPLC was performed, there were no peaks detected. Indicating that the protein was somehow lost during purification. This could be due to any number of factors. The protein may have an affinity for the matrix that was used during the separation process. Granule may have formed during the isolation process and clogged the matrix of the column. Or the protein could have degraded over the long transit time in the column, resulting in decreased concentration and lack of detection. 
A.

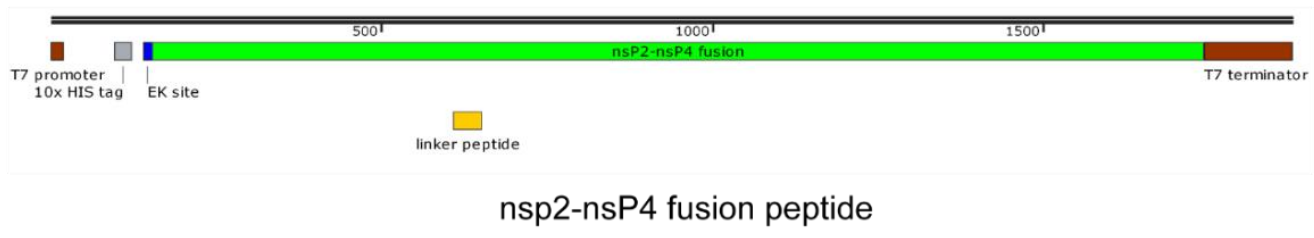

B.

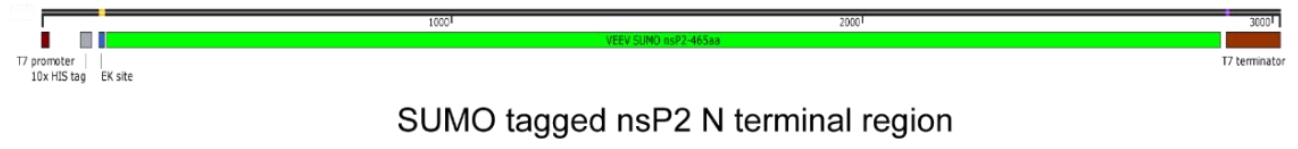

C.

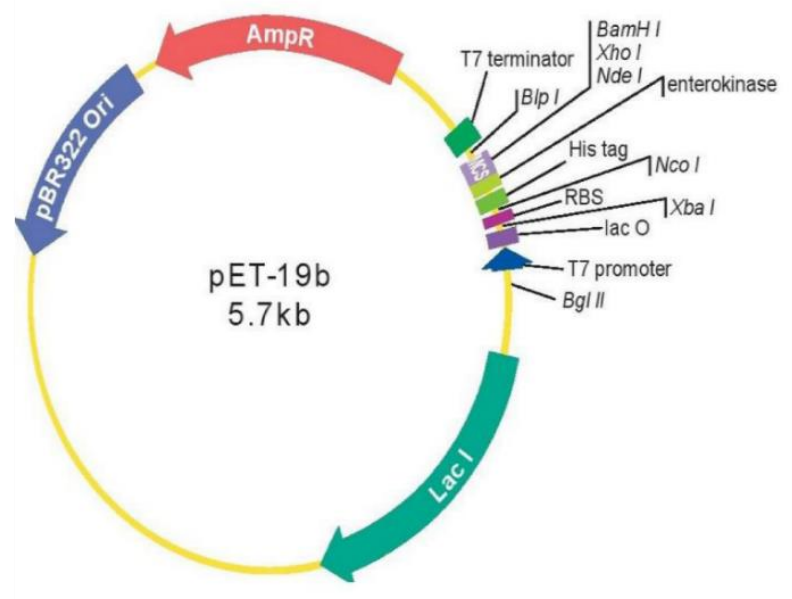

Figure 16. Expression constructs used for ectopic synthesis of VEEV nsP2. A) The fusion peptide combining $\mathrm{N}$ terminal portions of nsP2 and nsP2. B) The SUMO tagged $\mathrm{N}$ terminal nsP2 construct that produced soluble protein. C) The pET-19b vector backbone that was used for expression, map obtained from GenScript, from which these expression constructs were purchased. 

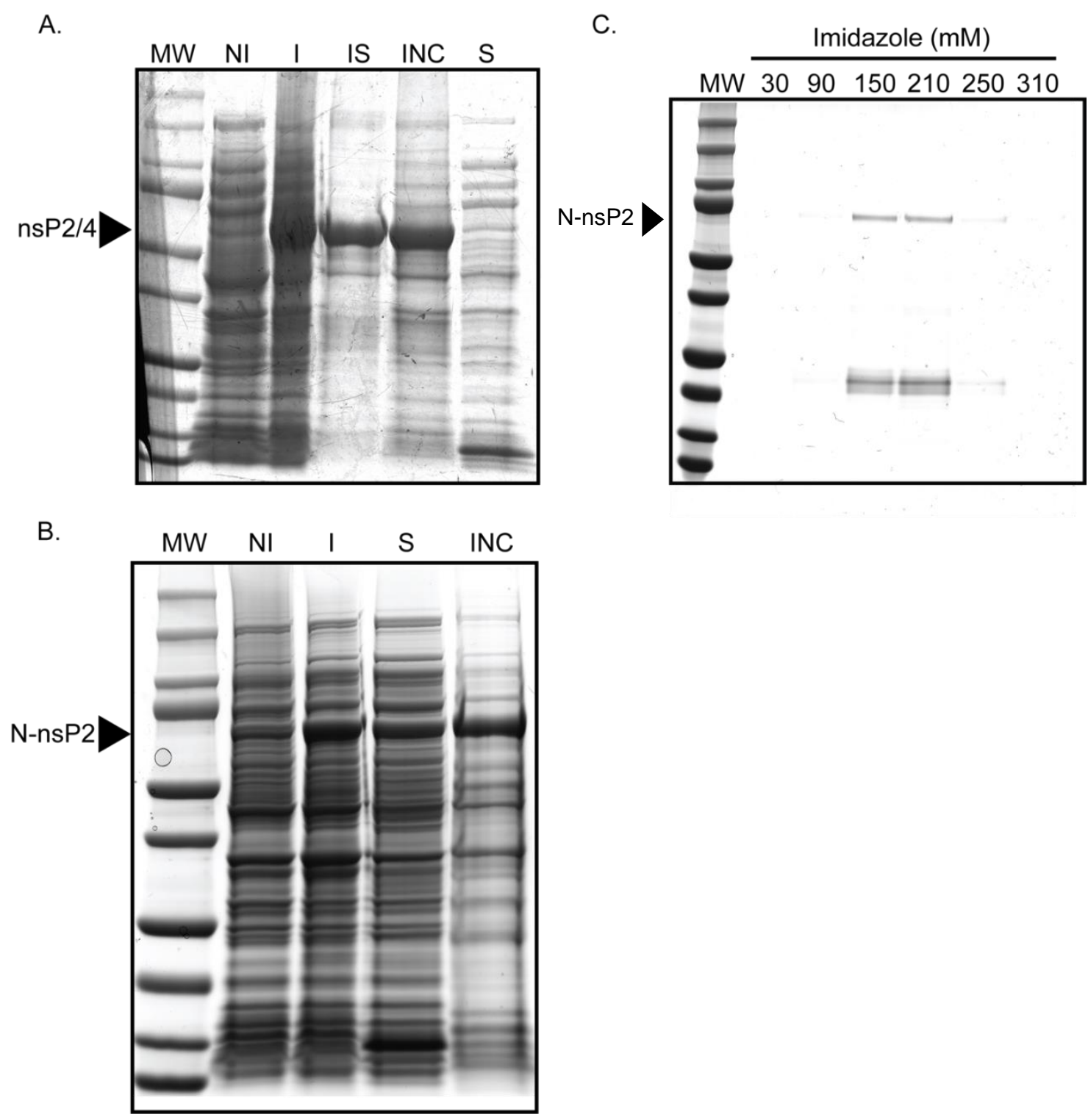

Figure 17. The expression and purification of VEEV nsP2 in bacterial cells. A) VEEV nsP2-nsP4 fusion protein was expressed in BL21(DE3) E. coli. The protein was insoluble and formed inclusion bodies. $\mathrm{MW}=$ molecular weight, $\mathrm{NI}=$ non induced, $\mathrm{I}=$ induced, IS= insoluble fraction, $\mathrm{INC}=$ inclusion bodies, $\mathrm{S}=$ soluble fraction. One representative image from three experiments. B) The $\mathrm{N}$ terminal 465 amino acids of nsP2 were expressed solubly using a SUMO tag. The protein expression was evenly 
split between the soluble fraction and the inclusion bodies. One representative image from 5 expression batches. C) Soluble nsP2-465 could be purified using Ni-NTA chromatography. Optimization of imidazole concentration was only performed once. 


\section{Discussion}

The isolation and use of viral proteins in biochemical and binding assays pose several challenges. Primarily, these proteins can often be difficult to isolate from infected cells as they occur at relatively low numbers, and like many proteins that are produced in eukaryotic systems, they are often difficult to produce using vector systems. However, based on previous work that had shown spherules could be successfully isolated from infected cells; we attempted to isolate the VEEV nsPs from infected cells directly. Unfortunately, this was unsuccessful.

The use of ultracentrifugation initially showed promise, as there was localization of activity from the isolated fraction, but this activity was lost after an additional round of purification across sucrose. Several methods were tried to improve this activity, such as the addition of viral RNA to serve as more template in the RNA synthesis reactions, changing the methods of RNA extraction, and altering the conditions of the synthesis itself, such as adding different amounts of radiolabel and rNTPs. None of these improved the activity of the fractions appreciably. While $\mathrm{CsCl}$ also did not result in good separation of the protein, there are other more specialized materials that can be used to as gradient material. One of these is iodixanol, a specialized sugar molecule that has been previously used in the isolation of biomolecules ${ }^{187}$. lodixanol is known to be gentle and is even used for preparation of live cells ${ }^{185}$. Thus the use of this material may result more preserved RNA synthesis activity and better preserve the structure of the isolated proteins. This would likely result in better maintenance of the enzymatic activity of these proteins, allowing for easier tracking during isolation. Gentler separation and isolation of the target proteins would also increase the concentration of the protein isolated from these infected cells.

As these attempts at isolating spherules proved impractical, the next attempt at detecting the interaction between the amidine compounds and the viral nsPs was carried 
out using click chemistry. The primary advantage of this method is that the viral proteins are labeled in the cell during infection, and that the detection of the labeled proteins is highly specific due to use of functional groups that do not naturally occur in biological systems. It was hoped that this experiment would result in specific labeling of viral proteins, and that this method could then be used to directly isolate these proteins whole cell lysate. The first attempt at this labeling, using gel electrophoresis and fluorescent labeling detection was unsuccessful. This is due to the fact that the viral proteins were not labeling efficiently enough to become apparent in the infected samples versus the uninfected control cells. One way that this could be improved is to treat cells later in infection. As time goes on, the synthesis of proteins in the cells shifts more towards the production of viral proteins. So treatment with L-AHA later in infection should more preferentially label viral proteins, allowing for more sensitive detection.

Due to the poor results from L-AHA labeling, alternative uses of click chemistry were investigated. It was found that this method has also been used in fluorescent microscopy by performing the click reaction directly in the cell monolayer. Working with our medicinal chemist collaborator who is responsible for the synthesis and development of the amidine scaffold compounds, she was able to manufacture both an active and an inactive compound that were appended with the functional groups necessary for the click reaction to occur. This allowed for the design of experiments where the click reaction could be performed in combination with staining for VEEV E protein and double stranded RNA. While it is true that these compounds are able to inhibit the formation of dsRNA due to their antiviral activities, if used for a short enough time, in this case only 30 minutes, we hypothesized that some replicative activity would remain. This experiment allows for the direct determination of compound localization in infected cells and can determine if they associate closely with the sites of viral replication. We hypothesized that the effective antiviral compound, 3260, would colocalize significantly with dsRNA, 
which is a readout for the localization of viral replication, and that the ineffective compound, 3261, would be dispersed throughout the cell. Unfortunately, these experiments proved inconclusive, as the primary difference that was seen between the two compounds was that the compound that lacked antiviral effects, compound 3261, appeared to be unable to enter cells at the same rate as the effective antiviral compound, 3260. Also, further experiments should use a different marker for the replicase complexes such as nsP2, as even with the short treatment times used there was a significant reduction in dsRNA staining.

While the results from these microscopy experiments were inconclusive, they resulted in the development of several new tools for further experiments. First, the click it reaction was successfully performed on a cell monolayer, and if the L-AHA or other metabolic labeling can be optimized, these can be combined to great effect. Second, these experiments led to the optimization of the use of a dsRNA antibody. This antibody is highly useful as it only stains those cells that are undergoing active viral replication, compared to cells that express viral protein. This antibody can also be used for colocalization studies to identify the loci of viral replication within infected cells.

The inconclusive results of the click experiments led to the work developing an expression system of VEEV nsP2 in E. coli. As outlined above, it was determined that the $\mathrm{N}$ terminal domain of the protein could be expressed in a soluble manner when fused to a SUMO tag. However, this took quite a bit of optimization of both the constructs and expression system. The first attempt was made using nsP2 and 4 fusion constructs. These constructs were readily expressed at high levels but were never able to be expressed in a soluble form, and refolding these proteins was also unsuccessful. Even in the Origami strain of $E$. coli which is designed for the expression of proteins that contain disulfide bonds and that typically don't fold correctly in expression vectors, this construct remained insoluble and disordered. 
This resulted an impasse until we incorporated the SUMO tag. This tag proved quite helpful in the expression of this protein. The resulting truncated protein constructs were produced in the soluble fraction of E. coli Rosetta at high concentrations. However, when this tag was used with full length nsP2 protein and not the truncated construct, it remained insoluble. If the full length $\mathrm{nsP} 2$ is required for future experiments, further optimization of this expression will need to be carried out. NsP4 was also expressed in this same system with a SUMO tag, and it too remained insoluble.

Additionally, the SUMO tagged nsP2 truncated construct had some issues with its purification. As shown, the complete construct can be readily purified using $\mathrm{Ni}$ chromatography. However, upon digestion with the SUMO protease, there is significant loss of protein sample, indicating that either the digestion, or the purification step performed to remove the tag need additional optimization. One way that this may be improved could be to incorporate a different protease site, such as an EK site, between the tag and nsP2. The use of a different cleavage method may result in a higher fidelity reaction. Different proteases also have different reaction conditions which may prove more suitable for our protein constructs, and result in less loss due to degradation. Also, a portion of the Ni chromatography purified protein was submitted for HPLC purification. Interestingly, despite a large protein input there were no peaks indicating proteins release from the column, and this was confirmed using the Bradford reaction. If HPLC is to be performed again, it is likely that the protein is binding to some part of the column, and so a different matrix types should be investigated. This protein construct may also have general issues of stability due to its nature as an incomplete truncated peptide. This would make slow purification schemes difficult. This should be examined further.

Lastly, the SUMO containing protein construct can be used as is for many different assays, such as surface plasmon resonance, which allow for the measurement of binding of the antiviral compounds and the protein construct. Pilot studies were 
performed using the OpenSPR platform from Nicoya, but unfortunately the sensitivity of the assay was poor, leading to inconclusive data. This is most likely due to the size of the antiviral molecules, as they are quite small, making the detecting of binding more difficult that with larger interactors. If these experiments are to be continued, then further work to increase the sensitivity of the assay should be performed. Such as by using various treatments to increase the amount of protein construct immobilized on the chip, or by immobilizing the small molecules on the chip and associating the protein construct.

\section{Conclusions}

Though this work attempting to detect and characterize predicted binding between the VEEV nsPs and the amidine scaffold was unsuccessful, it did lead to several useful experimental developments. First, the use of click chemistry in assays using both metabolic labeling of proteins and fluorescent microscopy was developed. While this chemistry did not prove useful for the experiments described here, these assays have many potential uses both monitoring the synthesis and localization of proteins as well as compound localization. There are also potential uses of this chemistry for directly purifying labeled proteins and compounds from cells and complex mixtures and extracts. Several additional tools were developed in tandem with these assays, including the use of a double stranded RNA antibody to track viral replication, and the novel amidine compounds that have click moieties that have potential to be used in many different experiments for both detection and purification. Second, an expression system for the $\mathrm{N}$ terminal domain of nsP2 was developed. This protein construct can be produced in large amounts as a soluble protein. This expressed protein is then readily purified by Ni chromatography. This system is ready for use in further biochemical assays. 


\section{CHAPTER 4}

THE USE OF AMIDINE COMPOUNDS TO CHARACTERIZE A REGION OF UNKNOWN FUNCTION IN VEEV NSP2 


\section{Overview}

While antiviral drugs are researched primarily for their potential uses as therapeutic compounds, these compounds can also be used in viral research in a variety of ways dependent on their mechanisms of action. One way that these molecules can be utilized is to probe viruses for novel biological functions that have yet to be described. In this chapter, the amidine family of compounds characterized in chapter two has been used to probe the biology of VEEV. In this chapter the isolation and sequencing of viruses that resist the effects of these compounds will be discussed. Then these isolates will be used to characterize the function of an $\mathrm{N}$ terminal region of the viral nsP2, which previously has not been characterized and lacks designated functions.

\section{Introduction}

Despite the apparent simplicity of many viruses, the study of their biology can be quite complex. This is due to several factors, such as the uniqueness of many viral proteins making the use of homology modeling and functional prediction difficult, as well as the fact that viruses are dependent on cellular processes for many parts of their life cycles, meaning that functional assays of the viral proteins may not reveal all of the functions that they perform. Many viruses are also incredibly efficient in the use of their genetic material, generating proteins that are multifunctional, with many interacting domains. The result of this is that even many well studied viruses have portions of their genomes and proteins that have yet to be functionally characterized. This includes alphaviruses. These viruses were first isolated in the $1930 \mathrm{~s}^{26,188-190}$ and have been thoroughly studied since, yet many parts of their proteins remain enigmatic. This 
difficulty in characterizing these regions necessitates the development of novel ways to probe viral biology in addition to traditional biochemical and virological methods.

One way that viruses are characterized in our lab is by using the antivirals that have been developed with our collaborators to perturb the viral life cycle. Due to their rapid mutations rates ${ }^{191}$ RNA viruses will often escape treatment with any antiviral compound, leading to generation of resistant populations that contain mutations in their genomes ${ }^{192}$. These mutant viral populations can then be plaque purified, and their genomes examined for mutations. In this work the Oxford Nanopore system has been utilized for sequencing of resistant viral isolates. This system is advantageous due to the small amount of starting material that it requires, as well as the fact that viral RNA can be sequenced directly.

Viruses that were resistant to the amidine family of compounds described in chapter 2 were isolated. After sequencing of these mutant populations, it was found that these viruses had mutations that were localized in the $\mathrm{N}$ terminal region of nonstructural proteins (nsP) 2. It was surprising to find mutations in this region because it currently lacks any predicted or described function.

NsP2 has been characterized as both the viral helicase protein as well as a cysteine protease $\mathrm{e}^{88,96,98,162,193}$. However, a large portion of the $\mathrm{N}$ terminal region remains undefined. There has been some progress in solving the structure of this protein using X-ray crystallography, with the $\mathrm{C}$ terminal protease region being solved in Venezuelan equine encephalitis virus (VEEV), and recently the $N$ terminal half of the protein was crystalized from chikungunya virus $(\mathrm{CHIKV})^{98,193,194}$. However there has yet to be a structure solved for the entire protein. The structure of $\mathrm{CHIKV}$ nsP2 indicates that the helicase and its accessory domains take up a large portion of the $\mathrm{N}$ terminal region. From $\mathrm{N}$ to $\mathrm{C}$-terminal these domains are: the $\mathrm{N}$-terminal domain, the stalk domain, $1 \mathrm{~B}$, 
Rec 1, Rec, 2, and the connector domain. The $1 \mathrm{~B}, \operatorname{Rec} 1$ and $\operatorname{Rec} 2$ domains are all involved in the RNA binding and helicase activity of the enzyme. However, the disordered $\mathrm{N}$-terminal domain and the stalk domain lack known fucntion ${ }^{98}$. Of the mutations that occur in nsP2 that result in resistance to compound treatment, the majority are in the $\mathrm{N}$-terminal and stalk domains, such as those at amino acid position 65 and 102, with some being in the far $\mathrm{N}$ terminal portion of the Rec $1 \mathrm{~A}$ helicase accessory domain, amino acid 116, far from the helicase active site, and arrayed primarily on the external faces of the protein.

For the purpose of this work, three viral isolates with mutations that occurred in nsP2 were selected. These mutants were characterized in a variety of classical virological methods, investigating their growth, RNA synthesis phenotypes, and protein expression. Initially, this characterization was carried out using baby hamster kidney cells, a cell line that has long be used in the study of alphaviruses due its high susceptibility and permissivity to infection ${ }^{195-197198}$..

After this initial characterization was completed the potential attenuation of these viruses was also examined in a neuronal cell model, SH-SY5Y cells. Neurons are a major cell type targeted by VEEV and are important in its pathology, and the effects of mutations in the nsPs may vary in these cells when compared to fibroblasts. SH-SY5Y cells are a human neuroblastoma cell line derived from metastatic cancer ${ }^{199}$. This cell model has an active viral response system while still being susceptible and permissive to infection with multiple viruses ${ }^{200-20237}$. These cells also have the advantage of being able to be differentiated into mature neurons ${ }^{203201}$. These differentiated cells are a reasonable facsimile of mature neurons in the brain ${ }^{203-205}$. This phenotype indicates that these cells provide a readily available, biologically relevant model of alphavirus infection of neurons. 


\section{Materials and Methods}

\section{Fibroblast cell culture and viral strains}

Baby hamster kidney (BHK) clone 21 cells (ATCC CCL-10) and Vero 76 (African Green monkey kidney fibroblasts) (ATCC® $\mathrm{CRL}-1587^{\mathrm{TM}}$ ) were maintained in Modified Eagle's Medium with Earle's Balanced Salt Solution and L-glutamine (MEM-E, Corning 10-010-CV) supplemented with 10\% fetal bovine serum (FBS) (Corning 35-011-CV). Cells were maintained at $37^{\circ} \mathrm{C}$ in humidified incubators with $5 \% \mathrm{CO}_{2}$. VEEV strain TC-83 (gift of Dr. Connie Schmaljohn, USAMRIID) was used for this study. Infections were carried out using a virus infection medium (Modified Eagle's Medium with Earle's Balanced Salt Solution with L-glutamine (Corning 10-010-CV), 25 mM HEPES, 10\% FBS)

\section{Undifferentiated SH-SY5Y cells}

SH-SY5Y cells are human neuroblastoma cells derived from metastatic cancer isolated from the bone marrow of a patient. Cells were grown on cell culture treated plates and maintained in at $37^{\circ} \mathrm{C} 5 \% \mathrm{CO}_{2}$. Cells were obtained from ATCC (CRL-2266). Cells were maintained in EMEM with 15\% FBS, 1x penicillin/streptomycin, $2 \mathrm{mM}$ glutamine. Cells were infected with the same media.

\section{Differentiation of SH-SY5Y cells}

Differentiation of SH-SY5Y cells into mature neurons has been well documented previously $200,203,204,206-208$. Cell were plated on untreated cell culture dishes (day 0). The following day (day 1) media was changed to differentiation media 1. Media was changed on days 3 and 5 . On Day 7 cells were split 1:1, all with differentiation media 1. Day 8 media was changed to differentiation media 2. Day 10 cells were split 1:1 onto dishes coated with extracellular matrix with differentiation media 2. Day 11 media was changed 
to differentiation media 3. Media was changed on days 14 and 17 with differentiation media 3. On Day 18 cells are mature and ready to use. Differentiation media 1: EMEM with 2.5\% FBS, 1x Penicillin/Streptomycin, $2 \mathrm{mM}$ glutamine, $10 \mu \mathrm{M}$ retinoic acid (STEMCELL technologies 72262). Differentiation media 2: EMEM, 1\% FBS, 1x Penicillin/Streptomycin, $2 \mathrm{mM}$ glutamine, $10 \mu \mathrm{M}$ retinoic acid. Differentiation media 3: Neurobasal media, 1x B-27, 20 mM KCl, 1x Penicillin/Streptomycin, 2 mM GlutaMAX, 50 $\mathrm{ng} / \mathrm{mL}$ brain derived neural growth factor (Sigma Aldrich B3795), 2mM dibutyryl cyclic AMP (Selleck Chemicals S7858), $10 \mu \mathrm{M}$ retinoic acid. Cells were infected in differentiation media 3.

\section{Plaque purification of resistant viral isolates}

Vero 76 cells were infected with VEEV TC-83 in the presence of $20 \mu \mathrm{M}$ CB1000905. Supernatant was collected and then used for plaquing with an agarose overlay, also with $20 \mu \mathrm{M}$ CB1000905. Plaques were picked and then placed in virus infection media to allow the virus to diffuse out of the agarose. These samples were then used to infect monolayers again under compound selection. This process was repeated a total of three times.

\section{Sequencing of whole viral genomes with the Minlon platform}

Vero 76 cells were infected at an MOI of ten with VEEV TC-83 and treated with compound CB1000905 at $20 \mu \mathrm{M}$. The media was aspirated, and TRI reagent was added at $300 \mu \mathrm{L}$ per well and the cells were homogenized by pipetting. The RNA was then isolated using a ZYMO mag bead RNA isolated kit according to manufacturer's instruction (R2101). Ribosomal RNA was depleted from the samples using an NEBNext rRNA depletion kit (NEB E6310S) according to manufacturer's instructions. This RNA was used as input for direct RNA sequencing using an Oxford Nanopore direct RNA 
sequencing kit (SQK-RNA002) according to manufacturer's instructions. Sequencing was performed using the Oxford Nanopore MinION platform. Sequences were analyzed using Integrative Genomics Viewer $209-211$.

\section{Viral growth kinetics}

BHK-21 cells were seeded into 6 well plates at a density of 400,000 per well. Plates were infected at an $\mathrm{MOI}$ of 0.05 with the indicated virus and placed on ice for one hour to synchronize the infection. Inoculum was $0.5 \mathrm{~mL}$. The cells were washed once with PBS and warm media was added to initiate the infection. The infection proceeded at $37^{\circ} \mathrm{C} 5 \% \mathrm{CO}_{2}$. Supernatant was collected at the indicated times and used for titration by plaque assay.

For infections of the SH-SY5Y, virus had to be diluted into media corresponding to the needs of either the undifferentiated or differentiated SH-SY5Y cells. Cells were grown in 24 well plates and infected at a volume of $300 \mu \mathrm{L}$. The virus was diluted into the corresponding media, for undifferentiated cells EMEM with 15\% FBS, 1x penicillin/streptomycin, $2 \mathrm{mM}$ glutamine was used. For differentiated cells differentiation media 3 was used. Cells were inoculated at either MOI 5 for single step growth analysis or $\mathrm{MOI} 0.05$ for multistep growth analysis. The cells were incubated at $37^{\circ} \mathrm{C} 5 \% \mathrm{CO}_{2}$ for one hour. The media was aspirated, and the cells washed with the corresponding media free of virus. Warm media was added, and this point was considered time zero for the infections. Supernatant was collected at the indicated times post infection, and these samples were used for plaque assay titration.

\section{Plaque assay titration}

Plaquing for titration of the viruses was carried out on Vero76 cells in 24 well plates. Cells were seeded at a density of 100,000 per well and allowed to grow overnight 
in complete media. Samples for titration were diluted in VIM (Modified Eagle's Medium with Earle's Balanced Salt Solution supplemented with 25 mM HEPES and 10\% FBS). and vortexed. $167 \mu \mathrm{L}$ of inoculum was used per well in 24 well plates. Media was aspirated from the cells and the dilute samples added and then incubated at $37^{\circ} \mathrm{C}, 5 \%$ $\mathrm{CO}_{2}$ for one hour. Plates were rocked initially and after 30 minutes. After one hour the cells were washed with PBS and overlay was added. For titration methyl cellulose overlay media was used (EMEM, 10\% FBS, 0.7\% w/v methyl cellulose, 1x GlutaMAX, 15 $\mathrm{mM}$ HEPES). These plates were then incubated at $37^{\circ} \mathrm{C} 5 \% \mathrm{CO}_{2}$ for three days, and then stained and fixed with a solution of $2 \%$ paraformaldehyde, $0.8 \%$ crystal violet in ethanol, dissolved in PBS.

\section{Plaque size analysis}

To measure the relative size of plaques of the viral isolates the protocol above was modified. Vero 76 cells were seeded at a density of 400,000 cells per well in six well plates. Samples were diluted in VIM and $668 \mu \mathrm{L}$ of inoculum was added per well. Cells were incubated and washed as above. One $\mathrm{mL}$ of overlay media was added per well: $1 \mathrm{x}$ EMEM (Gibco 11430-30), 0.6\% agarose (MP Biomedicals 952012), 0.22\% sodium bicarbonate (Gibco 25080-094), 1x GlutaMAX, 1x nonessential amino acids (Gibco 11140050), 1x penicillin/streptomycin (Gibco 15140122), 15mM HEPES, 10\% FBS. The plates were incubated at $37^{\circ} \mathrm{C} 5 \% \mathrm{CO}_{2}$ for 2 days and fixed with $4 \%$ paraformaldehyde at $4{ }^{\circ} \mathrm{C}$. The cells were then stained with $2 \%$ paraformaldehyde, $0.8 \%$ crystal violet in ethanol, dissolved in PBS. The plates were scanned, and the images used for plaque size analysis and counting using the viral plaque plugin for ImageJ/FIJ|212.

\section{Isolation of viral RNA for qRT-PCR characterization}


BHK cells were infected at an MOI of ten. The cells were placed on ice for one hour to synchronize infection. The cells were washed with PBS and then warm media was added to initiate infection. The cells were placed at $37^{\circ} \mathrm{C} 5 \% \mathrm{CO}_{2}$ for the indicated times. At the indicated times, the media was aspirated, and TRI reagent (ZYMO R2050) added at $300 \mu \mathrm{L}$ per well and the cells were homogenized by pipetting. The RNA was then isolated using a ZYMO mag bead RNA isolated kit according to manufacturer's instruction (\#R2101).

\section{cDNA synthesis for qRT-PCR analysis}

RNA samples were isolated as described above. Two master mixes were made as follows:

Master Mix 1:

\begin{tabular}{|l|l|}
\hline Reagent & Amount per one reaction $(\mu \mathrm{L})$ \\
\hline $10 \mathrm{mM}$ dNTPs (Promega U1515) & 1 \\
\hline $\begin{array}{l}\text { Custom primer }(10 \mathrm{pmol} / \mu \mathrm{L}) \text { or } \\
\text { random hexamer }(40 \mathrm{pmol} / \mu \mathrm{L})\end{array}$ & 1 \\
\hline Nuclease free water & 4 \\
\hline
\end{tabular}

Four $\mu \mathrm{L}$ of RNA was added to this master mix and the reaction was heated at $65 \mathrm{C}$ for 5 minutes. The reaction was then chilled on ice for 5 minutes.

Master Mix 2:

\begin{tabular}{|l|l|}
\hline Reagent & Amount per one reaction $(\mu \mathrm{L})$ \\
\hline $\begin{array}{l}\text { 5X reverse transcriptase buffer } \\
\text { (ThermoFisher EP0743) }\end{array}$ & 4 \\
\hline SUPERaseln (Ambion AM2694) & 0.5 \\
\hline $\begin{array}{l}\text { Maxima H Minus Reverse } \\
\text { Transcriptase (ThermoFisher EP0743) }\end{array}$ & 0.5 \\
\hline Nuclease free water & 5 \\
\hline
\end{tabular}


Ten $\mu \mathrm{L}$ of master mix two was added to each reaction after chilling. The reaction as gently mixed and then submitted to the following protocol for cDNA generation and thermal degradation of RNA:

1. $25^{\circ} \mathrm{C}$ for $5 \mathrm{~min}$.

2. $50^{\circ} \mathrm{C}$ for $60 \mathrm{~min}$.

3. $85^{\circ} \mathrm{C}$ for $5 \mathrm{~min}$.

4. $4^{\circ} \mathrm{C}$ hold

This cDNA was then used for further analysis in qRT-PCR analysis as described below.

\section{Strand-specific quantitative real-time PCR (qRT-PCR) of VEEV RNA}

Detection of positive and negative sense, genomic viral RNA was carried out using a strand-specific qRT-PCR method adapted from Plaskon et al ${ }^{165}$. Briefly, cDNA was generated using tagged primers for detecting positive-sense and negative-sense RNA. The generated cDNA was then used in qRT-PCR using TaqMan chemistry with a strand-specific primer set.. A fluorescent probe was used for both analyses. PCR reaction was performed using Fast Advanced TaqMan master mix (Applied biosystems 4444557). PCR cycles were performed according to manufacturer's instructions. The primer sequences are given in the following table. Lowercase sequences are additional sequence added for primer identification, sequences in italics are specific for viral RNA, and sequences in bold were used to identify only those cDNA sequences that were produced due to primer binding. All primers and probes were custom ordered from IDT.

\begin{tabular}{|c|c|}
\hline Primer & Sequence \\
\hline nsP1 positive Tag & GGCAGTATCGTGAATTCGATGC CTGACCTGGAAACTGAGACTATG \\
\hline $\begin{array}{l}\text { nsP1 negative } \\
\text { Tag }\end{array}$ & GGCAGTATCGTGAATTCGATGCGGCGACTCTAACTCCCTTATTG \\
\hline $\begin{array}{l}\text { nsP1 positive } \\
\text { FWD }\end{array}$ & aataaatcataa CTG ACC TGG AAA CTG AGA CTA TG \\
\hline
\end{tabular}




\begin{tabular}{|c|c|}
\hline $\begin{array}{l}\text { nsP1 positive } \\
\text { REV }\end{array}$ & aataaatcataa GGC AGT ATC GTG AAT TCG ATG C \\
\hline $\begin{array}{l}\text { nsP1 negative } \\
\text { FWD }\end{array}$ & aataaatcataa GGC GAC TCT AAC TCC CTT ATT G \\
\hline $\begin{array}{l}\text { nsP1 negative } \\
\text { REV }\end{array}$ & aataaatcataa GGC AGT ATC GTG AAT TCG ATG C \\
\hline nsP1 probe & /56-FAM/TCC GTC AAC /ZEN/CGC GTA TAC ATC CTG /3IABkFQ \\
\hline
\end{tabular}

\section{qRT-PCR analysis of subgenomic RNA synthesis}

RNA samples from six hours post infection were used. cDNA was generated from RNA isolated from infected cells as described above using random hexamer. This cDNA was then used in a multiplex qRT-PCR with the following primers and TaqMan probes with TaqMan Fast Advanced master mix. All primers and probes were custom ordered from IDT.

\begin{tabular}{|l|l|}
\hline Primer & Sequence \\
\hline Capsid FWD & GGACGACCCATTCTGGATAAC \\
\hline Capsid REV & CGTTCCACATGACGACTGAA \\
\hline Capsid Probe & /5SUN/TCCTTCATT/ZEN/CACACCTCCCAGCAC/3IABkFQ/ \\
\hline nsP1 FWD & CTGACCTGGAAACTGAGACTATG \\
\hline nsP1 REV & GGCGACTCTAACTCCCTTATTG \\
\hline nsP1 Probe & /56FAM/TCCGTCAAC/ZEN/CGCGTATACATCCTG/3IABkFQ \\
\hline
\end{tabular}

The double delta CT was calculated and used to generate the relative gene expression that is shown.

\section{Immunoblotting}

Whole cell lysate from infected cells was collected by directly lysing cells with Laemmli buffer in the plate $(2 \%$ SDS w/v, 10\% glycerol w/v, $120 \mathrm{mM}$ Tris- $\mathrm{HCL} \mathrm{pH}=6.8$, bromophenol blue $)^{213}$. The resulting lysate was homogenized by pipetting. Samples were loaded into gradient gels for SDS-PAGE (GenScript M00656). Samples were run at $170 \mathrm{~V}$ until the dye front was run off of the gel. Protein was transferred to a PVDF (Biorad 1620177) membrane using methanol Tris-glycine buffer and a semi-dry blotting 
apparatus, $18 \mathrm{~V}$ for 35 minutes. Membranes were washed once and blocked with $5 \%$ dry milk in TBS. The membranes were washed five times in TBS-T and then stained with primary antibody overnight at $4{ }^{\circ} \mathrm{C}$. Anti-nsP2 E3 antibody (purified custom polyclonal antibody from GenScript) and anti-E protein polyclonal antibody (BEI) were both used at a 1:1000 dilution in TBS-T. The membranes were then washed five times with TBS-T and stained with secondary antibody diluted in TBS-T. Anti-rabbit HRP (Santa Cruz Biotechnology Sc-2054) was used to detect the nsP2 antibody and is used at a final concentration of $0.01 \mathrm{mg} / \mathrm{mL}$. Anti-goat HRP (SeraCare) was used to detect the $\mathrm{E}$ protein and was used at a concentration of 1:10,000. Actin was stained directly using an HRP conjugated antibody (CellSignal 8H10D10) final dilution 1:10,000. The secondary antibody staining is performed for one hour at room temperature. The membranes are washed six times in TBS-T and developed in ECL reagent for five minutes at room temperature (ECL reagent A: $2.5 \mathrm{mM}$ luminol, $400 \mathrm{mM}$ P-coumaric acid, $100 \mathrm{mM}$ Tris$\mathrm{HCL} \mathrm{pH}=8.5$. Reagent $\mathrm{B}: 0.02 \% \mathrm{H}_{2} \mathrm{O}_{2}, 100 \mathrm{mM}$ Tris- $\mathrm{HCl} \mathrm{pH}=8.5$. Mix $\mathrm{A}$ and $\mathrm{B}$ one to one for development). The images were collected using an Azure imaging system for detection of chemiluminescence. For staining of the E protein, the membranes previously stained for actin and nsP2 were stripped in acid stripping buffer (1\% w/v SDS, $25 \mathrm{mM}$ glycine $\mathrm{HCL} \mathrm{pH=2)}$ for 30 minutes at room temperature with rapid agitation, and washed twice with PBS, and once with TBS-T before proceeding with blocking and staining.

\section{Statistics}

Statistics were performed in GraphPad Prism version 9. Unless otherwise indicated significance was calculated using ANOVA with Dunnett corrections for multiple comparisons. Error is reported as standard deviation. Graphs represent mean values of the indicated number of experiments. 


\section{Results}

\section{Compound resistant viral isolates of VEEV have mutations in nsP2}

Previous work with the antiviral compounds in our lab has identified the locations of mutations that occur in viral isolates that resist compound treatment which were isolated via plaque purificaiton ${ }^{148}$. Three isolates were selected that were known to have mutations in nsP2, to focus on characterizing unknown activities of this protein. After the initial selection, these viruses were submitted to whole genome sequencing to determine if there were any additional mutations in their genomes that had not been previously discovered. This process utilizes a direct RNA sequencing method developed by Oxford Nanopore for use with their MinION platform.

It was found that mutations occurred in multiple locations in each of the selected viral isolates. Two of the isolates had single mutations in nsP2 at Y102 and D116. Both of these isolates also had point mutations in their $E$ proteins. The third selected isolate had two mutations in nsP2 at both $\mathrm{Y} 65$ and $\mathrm{Y} 102$. This isolate also had a single point mutation in the subgenomic promoter region. While this is a noncoding region in the viral RNA it is highly important due to its regulation of subgenomic RNA synthesis. This led to the hypothesis that this mutant isolate is likely to have significant alterations to its expression of the structural proteins. The nucleotide sequence of the area of nsP2 that is mutated is shown in Figure 18, nucleotide changes are shown in red, and the position of the nucleotide is indicated. Maps of the viral genome are shown in Figure 19, with each of the mutations indicated. Table 3 lists the selected isolates with their mutations. 
TC-83 5' gccgtggaaccatalccatggtaaagtagtggtgccagagggacatgcaatacccgtccaggactttcaagctctgagtgaaagtg 82_11_24 5' gccgtggaaccatajccatggtaaagtagtggtgccagagggacatgcaatacccgtccaggactttcaagctctgagtgaaagtg 81_12_24 5' gccgtggaaccataccatggtaaagtagtggtgccagagggacatgcaatacccgtccaggactttcaagctctgagtgaaagtg 6_13_25 5' gccgtggaaccatgjccatggtaaagtagtggtgccagagggacatgcaatacccgtccaggactttcaagctctgagtgaaagtg 1843

TC-83 ccaccattgtgtacaacgaacgtgagttcgtaaacaggtalctgcaccatattgccacacatggaggagcgctgaacactgatga 3' 82_11_24 ccaccattgtgtacaacgaacgtgagttcgtaaacaggtalcctgcaccatattgccacacatggaggagcgctgaacactalatga 3' 81_12_24 ccaccattgtgtacaacgaacgtgagttcgtaaacaggtg|cctgcaccatattgccacacatggaggagcgctgaacactogatga 3' 6_13_25 ccaccattgtgtacaacgaacgtgagttcgtaaacaggtg cctgcaccatattgccacacatggaggagcgctgaacactgatga 3' 1954 1995

Figure 18. Single nucleotide polymorphisms of the selected viral isolates in N-nsP2. The sequences of VEEV TC-83 as well as the three selected mutant isolates are shown. The mutated nucleotides are shown in red and the poisitons boxed in. The nucloetide position is below the selected bases. All of the selected amino acid changes are due to single nucleptide polymoorphisms. 

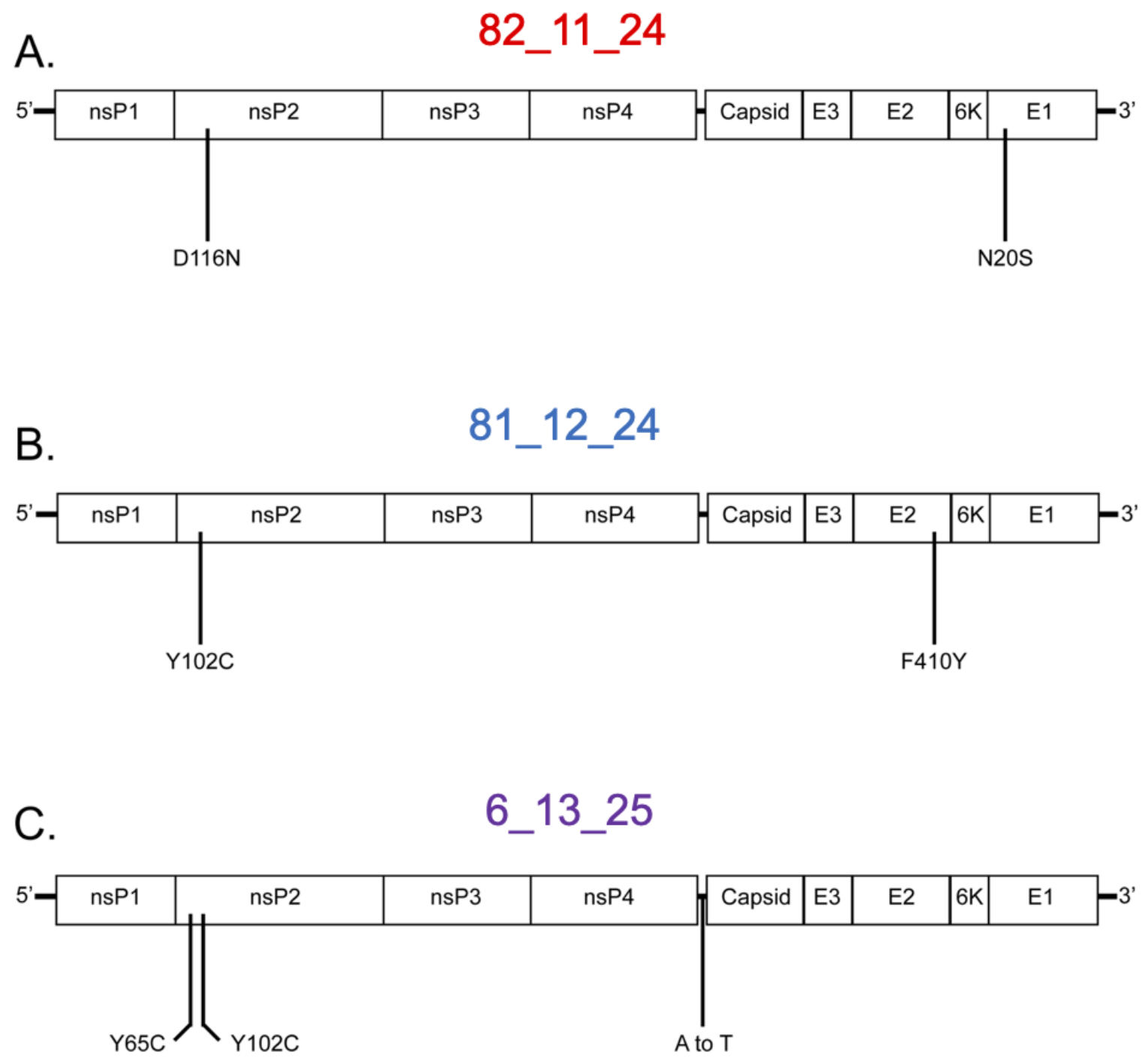

Figure 19. Maps of the full length genomes of the selected mutant viruses. A) Simplified map of isolate 82_11_24. Sequencing found a mutation, D116N located in the $1 \mathrm{~B}$ domain in nsP2, and there was an additional point mutation in E1. B) Simplified map of isolate 81_12_24. Sequencing found a mutation, Y102C located in the stalk domain in nsP2, and there was an additional point mutation in E2. C) Simplified map of isolate 6_13_25. Sequencing found two mutations, Y65C in the N-terminal domain and Y102C 
in the stalk domain in nsP2. There was an additional SNP in the region corresponding to the subgenomic promoter. 


\begin{tabular}{|c|c|c|c|c|c|c|c|}
\hline $\begin{array}{l}\text { Amino } \\
\text { Acid } \\
\text { Position }\end{array}$ & $\begin{array}{c}\text { nsP2 } \\
\text { Y65 }\end{array}$ & $\begin{array}{l}\text { nsP2 } \\
\text { Y102 }\end{array}$ & $\begin{array}{l}\text { nsP2 } \\
\text { D116 }\end{array}$ & $\begin{array}{l}\text { Sub } \\
\text { Prom } \\
7531 \text { A }\end{array}$ & $\begin{array}{c}E 2 \\
F 410\end{array}$ & $\begin{array}{c}\text { E1 } \\
\text { N20 }\end{array}$ & $\begin{array}{l}\text { Short } \\
\text { name }\end{array}$ \\
\hline TC-83 & --- & --- & --- & --- & --- & --- & --- \\
\hline $82 \_11 \_24$ & --- & --- & $\mathrm{N}$ & --- & --- & $S$ & 1124 \\
\hline 81 12_24 & --- & C & --- & --- & $\mathrm{Y}$ & --- & 1224 \\
\hline 6_13_25 & $\mathrm{C}$ & $\mathrm{C}$ & --- & $\mathrm{T}$ & --- & --- & 1325 \\
\hline
\end{tabular}

Table 3. Selected resistant viral isolates. Mutations are as indicated. 


\section{Compound resistant mutant viruses displayed delayed growth in a fibroblast cell model.}

The three mutant viruses were expanded under compound selection to ensure that the mutations were maintained through passaging. BHK cells were then infected at an $\mathrm{MOI}$ of 0.05 and a growth kinetics assay was performed using plaque assays to determine viral titer. It was hypothesized that isolate 6_13_25, with the subgenomic promoter mutation, would have a significant reduction in growth due the predicted effect this mutation would likely have on structural protein expression. However, the mutant isolates did not demonstrate a reduction in maximum titer compared to TC-83, Figure 20 A. Instead each virus eventually reached and maintained a titer that was similar to the parental strain. These mutations did, however, result in delayed growth compared to the parental control TC-83. Isolates 82_11_25 and 81_12_24 both had delayed viral production, but quickly caught up to TC-83. 6_13_25 was the most significantly delayed and took the longest to reach parity with TC-83, Figure $20 \mathrm{~A}$. These results show that the mutations found in the resistant viral isolates lead to a growth delay, with slower infection, and production of infectious virus, but not a growth defect in this non-selective cell line.

While performing the plaque assays for the growth kinetics analysis, plaque morphology changes were noted in isolate 6_13_25. To examine this in greater detail, agarose plaque assays in 6 well plates were used, Figure $20 \mathrm{~B}$. This analysis showed that 6 _13_25 did in fact have smaller plaques than the other isolates or TC-83, Figure 20 C. This is not surprising, as the structural proteins, and capsid in particular, are very important to the biological activities of alphaviruses and are under control of the subgenomic promoter ${ }^{7,55}$. The capsid protein is also very important in inducing cytopathic effect, detected phenotype in plaque assays ${ }^{80}$. These plaques also had a much tighter 
distribution compared to TC-83 or the two single mutants. This phenotype indicates that isolate 6_13_25 is significantly attenuated compared to TC-83 and is less fit for cell to cell spread or cytopathic effect, in addition to the growth defect that it shares with the isolates that lack the subgenomic promoter mutation. Isolates 81_12_24 and 82_11_24 have more moderate attenuations. 
A.

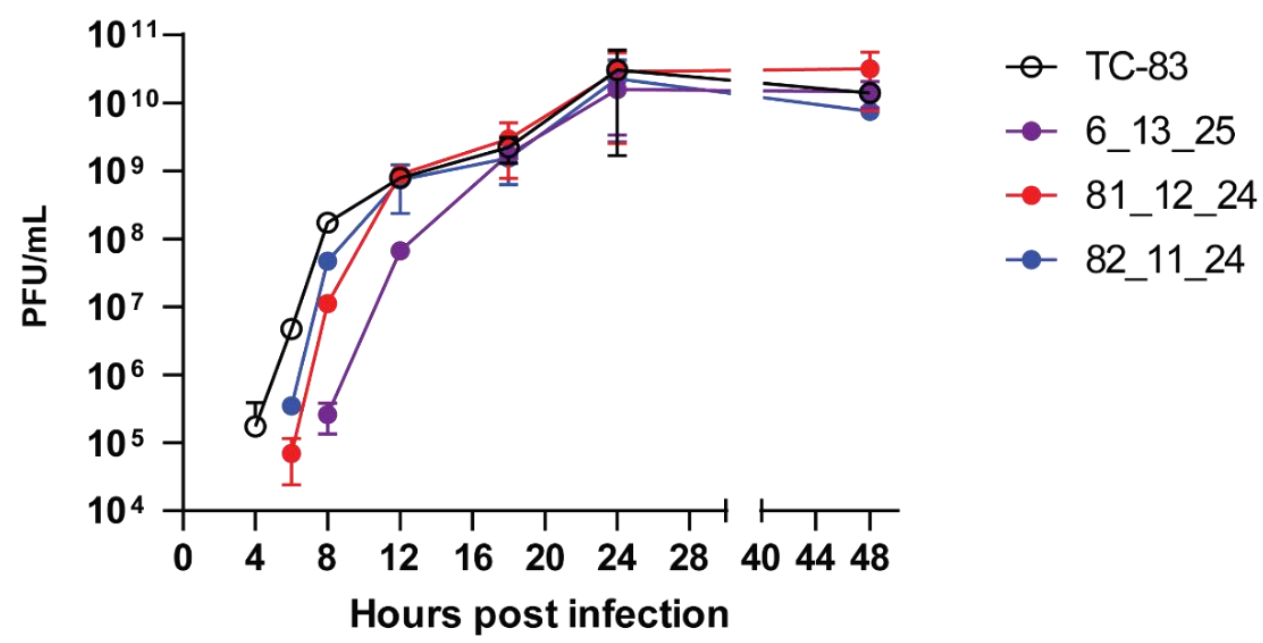

B.

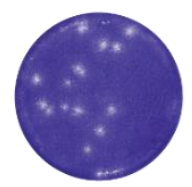

TC-83

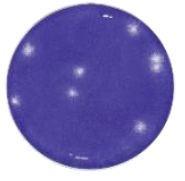

81_12_24
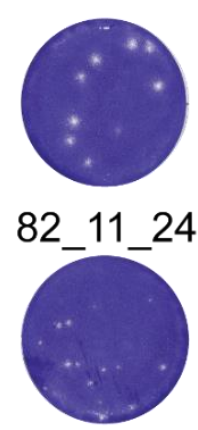

6_13_25

C.

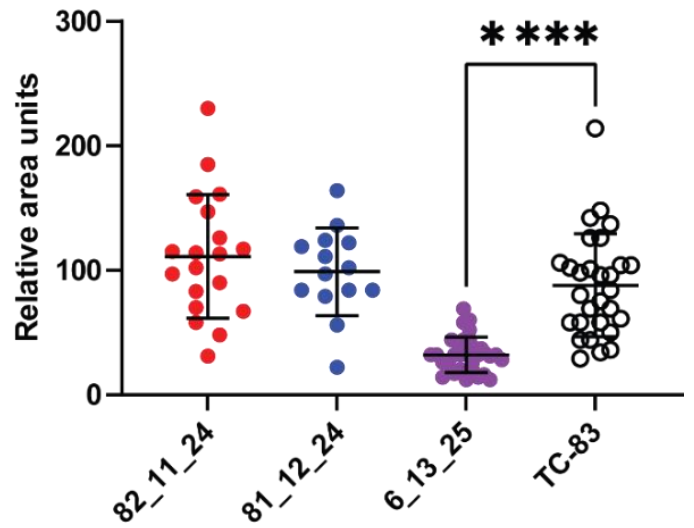

Figure 20. Mutant viral isolates show delayed growth in a fibroblast cell mode, and isolate 6_13_25 has decreased plaque size. Three mutant viruses were selected with the indicated mutations in nsp2. BHK cells were infected at an $\mathrm{MOI}$ of $0.05 \mathrm{~A}$ ) All of the mutant viruses showed a delay in the production of new infectious virus as measured by plaque assay. Isolate 6_13_25 showed a larger delay than either of the two single mutants. Kinetics data combined from two experiments, three biological replicates per time point per experiment. $48 \mathrm{HPI}$ was only collected from one experiment $\mathrm{B}$ ) Representative images of plaque morphology from each of the viruses using an agar 
overlay and staining the monolayers with crystal violet. Representative of three experiments. C) Quantification of the relative size of the plaques from each virus. The double mutant shows plaques that are significantly smaller than those of the parental TC-83 strain. The single mutants show no change. Graph is from one representative experiment of three. Three biological replicates per experiment. $\mathrm{P}<0.0001$ as calculated by one way ANOVA. 


\section{Compound Resistant Viral Isolates Show Alterations in Their RNA synthesis profiles}

NsP2 is highly important in the RNA synthesis of alphaviruses, acting as the RNA helicase of these viruses and having RNA binding activity (described in detail in chapter 1). Having noted a delay in growth of these resistant isolates, it was hypothesized that this growth delay resulted from a defect in viral RNA synthesis, likely being mediated by the mutations that occur in $\mathrm{N}$ terminal region of $\mathrm{nsP2}$.

Alphaviruses produce both positive and negative-sense RNA for replication ${ }^{82}$. These strands are both synthesized by the nonstructural proteins, but this synthesis is tightly controlled $58,59,61,160,214$. Thus, the synthesis of these two strands was examined separately, using a modified RT-PCR that is able to discriminate between the two different of polarities of RNA.

It was found that both isolate 81_11_24 and 6_13_25 had delayed production of positive-sense RNA, Figure 21. This was similar to growth kinetics phenotypes. With the detection of the positive sense RNA being delayed early in infection and catching up to the parental virus strain by 8 hours post infection. That both of these isolates show this delay indicates a likely involved of the $\mathrm{N}$ terminal domain of VEEV nsP2, as isolate 81_11_24 lacks the significant mutation in its subgenomic promoter. However, only isolate 6_13_25 showed a significant delay in the synthesis of negative-sense RNA, Figure 22. This indicates a likely involvement of nsP2 Y65C in the synthesis of negativesense RNA as only this isolate contains this mutation, and this synthesis should not be affected by the subgenomic promoter. 
The relative expression of the subgenome was also examined, Figure 23 . This was quantified by comparing the ratio of subgenomes to genomes in each of the viral isolates and TC-83. It was hypothesized that isolate 6_13_25 would have significant alterations in subgenomic RNA synthesis levels due to the mutation in the subgenomic promoter. While the other two isolates have point mutations in their glycoproteins, these proteins are not involved in RNA synthesis, so it was unlikely that the synthesis of the RNA would be effected. It was found that isolate 6_13_25 had greatly reduced subgenomic RNA expression, producing virtually no subgenomes at the time point examined, and the two other isolates displayed similar ratios to the parental TC-83 strain, Figure 23. 
2 HPI

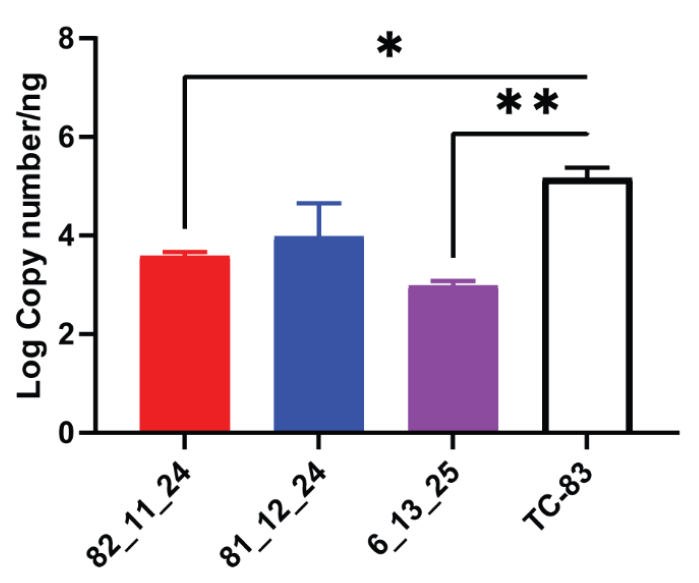

$4 \mathrm{HPI}$

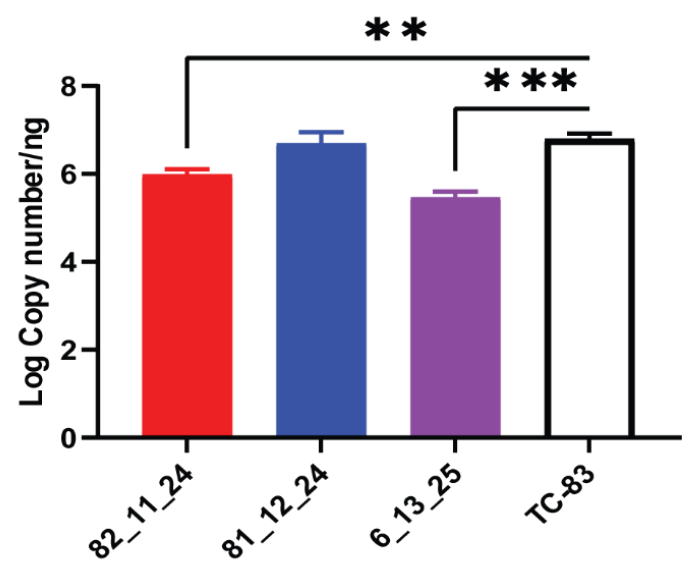

\section{$8 \mathrm{HPI}$}

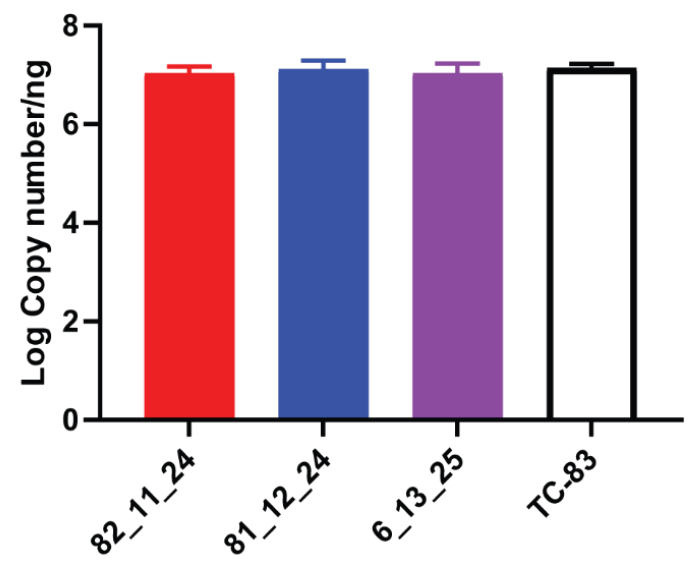

Figure 21. Mutant viral isolates have a delay in positive-sense RNA synthesis. Strand specific qRT-PCR was performed at the indicated times post infection. Isolates 82_11_24 and 6_13_25 both show reduced levels of positive-sense RNA at two and four hours post infection. This difference is lost at eight hours post infection, by which time every strain has reached the same level of RNA Data from one representative experiment of three. Three biological replicates per experiment. ${ }^{*} p<0.05,{ }^{* *} p<0.01$, ${ }^{\star * *} p<0.001$ as measured by one way ANOVA. 
2 HPI

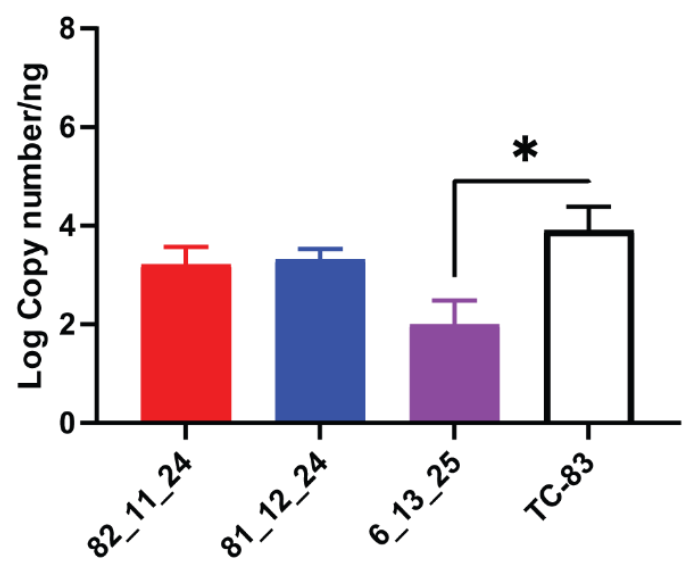

4 HPI

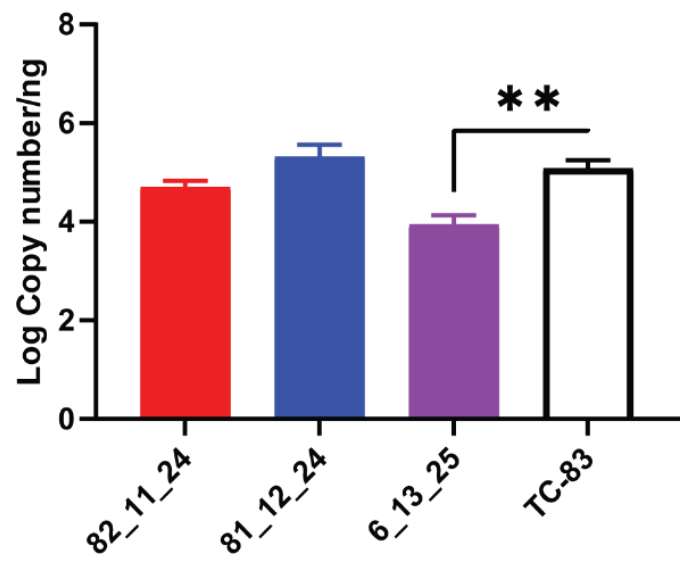

8 HPI

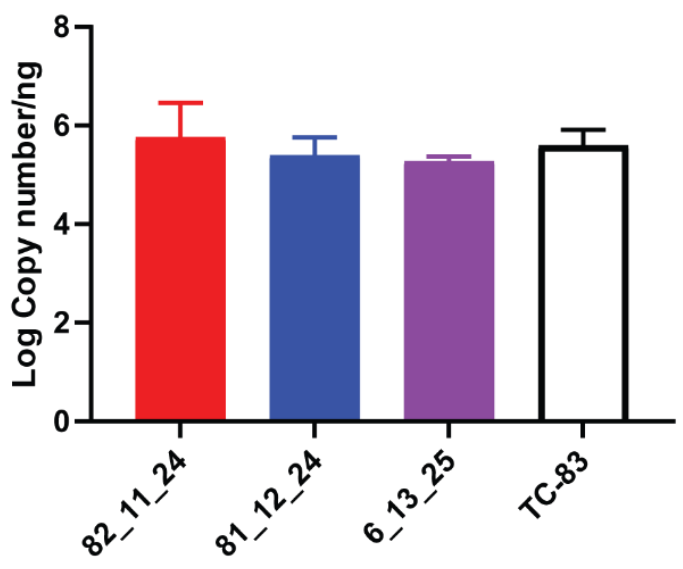

Figure 22. Isolate 6_13_25 has a delay in negative-sense RNA synthesis. Strand specific qRT-PCR was performed, and the amount of negative sense viral RNA quantified. Isolate 6_13_25 was found to have reduced negative sense RNA at two and four hours post infection. This difference was no longer present at eight hours post infection. Data from one representative experiment of three. Three biological replicates per experiment. ${ }^{*} p<0.05,{ }^{* *} p<0.01$ as measured by one way ANOVA. 


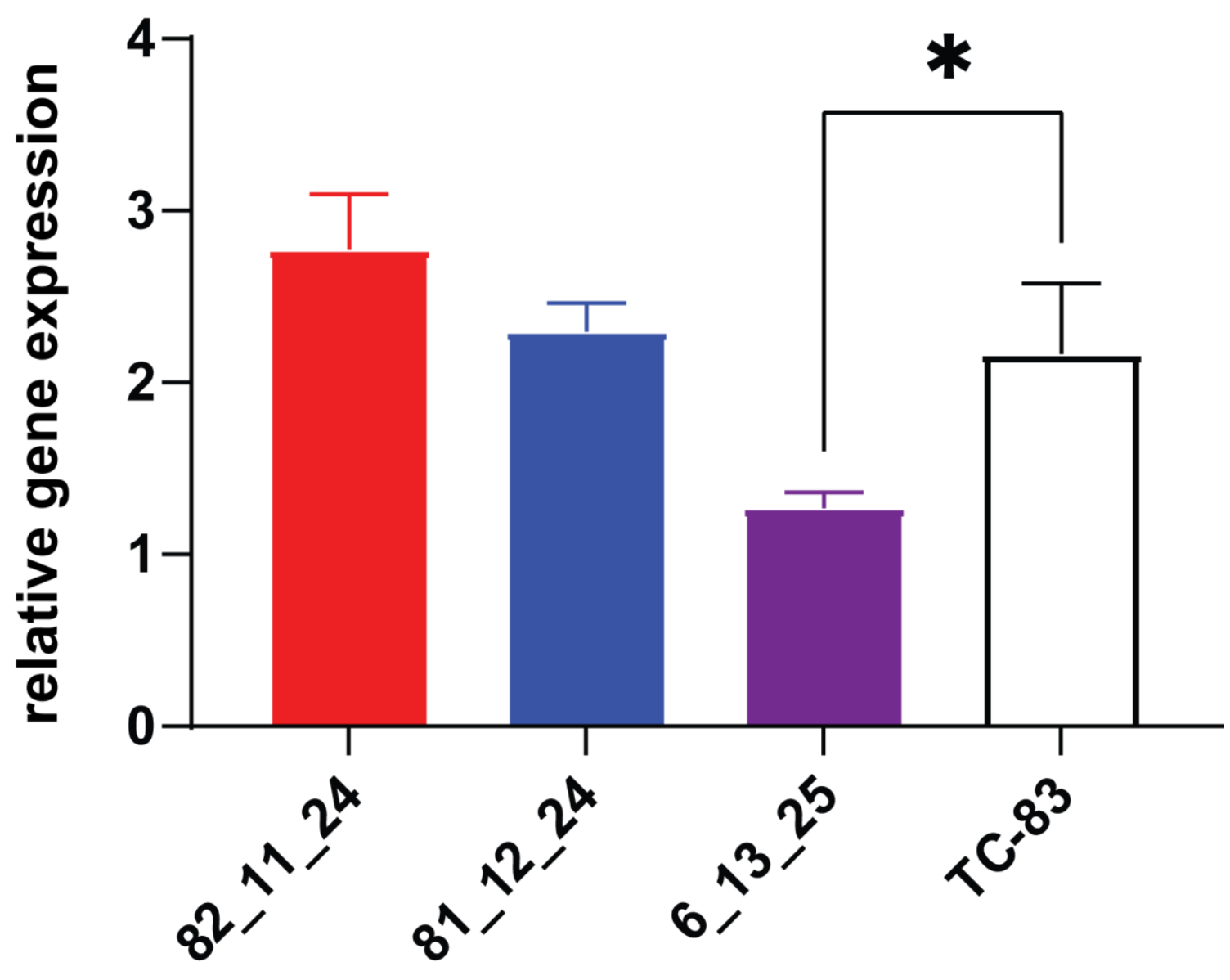

Figure 23. Relative expression of the subgenomic RNA compared to genomic RNA. Expression of capsid RNA was quantified relative to the expression of nsP1. This relative expression was then compared between the mutant viral isolates and TC-83. Only isolate 6_13_25 is significantly different from TC-83 with a ratio of one half that of the parental strain, indicating there is little to no subgenomic expression at this time. Data from one experiment, three biological replicates. ${ }^{*} p<0.05$. 


\section{Viral Isolates with mutations in nsP2 have altered protein expression profiles}

Having investigated both the growth and RNA synthesis profiles of these compound resistant viral isolates, the next step was to examine their protein expression. The finding that two of these isolates had altered RNA synthesis led to the hypothesis that they would have similar delays in the expression of the viral proteins, indicating that a delay in replication of the viral RNA was leading to the delay seen in viral growth kinetics. In addition, it was expected that there would be marked decrease in the structural proteins of isolate 6_13_25 due to large decrease in its transcription of the subgenomic RNA. While there was not a statistically significant delay in RNA synthesis in isolate 81_12_24, it did have a delay in growth kinetics and displayed a trend of reduce positive-sense RNA synthesis at early times points, so it was expected to show a delay in protein synthesis as well.

To examine viral proteins synthesis, immunoblotting was performed. Nsp2 was used as a marker of the expression of the viral nonstructural gene and has been previously used by our lab in this manner ${ }^{66}$. The $\mathrm{E}$ protein was used as a maker for the expression of the viral structural protein, the antibody used here stains both the $\mathrm{E} 1$ and E2 proteins. Beta actin was used as a loading control, and its intensity was used to quantify the relative expression of the viral proteins across the different samples.

Protein expression was examined in BHK cells, which were infected at an $\mathrm{MOI}$ of ten and then lysed at the indicated times post infection. Representative blot images are shown in Figure 24. NsP2 had delayed expression in all of the resistant isolates, and was detected at lower levels than TC-83 until eight hours post infection, at which time the expression of nsP2 begins to match that of the parental strain, Figure $25 \mathrm{~A}$. At ten 
hours post infection, the slowest of the mutant viruses, isolate 6_13_25, has increased levels of nsP2 compared to TC-83. If 6_13_25 is less cytotoxic, then there would be more live infected cells at this later time point post infection, which would leave more infected cells to by lysed and used for detection. At this time post infection there was no staining of any forms of the nonstructural polyprotein.

When examining the expression of the $\mathrm{E}$ proteins, the hypothesis about isolate 6_13_25 was correct, this isolate had a significant reduction in expression of the protein at 10 hours post infection, Figure $25 \mathrm{~B}$. This was true for all cleavage forms of the $\mathrm{E}$ proteins. It was unclear from their sequences if the other two isolates would have any alterations in their expression of the structural proteins. While both 82_11_24 and 81_12_24 had statistically significant differences in their expression of the E proteins at ten hours post infection, these differences are small in magnitude and not likely biologically significant. None of the isolates had significant changes in their expression at 8 hours post infection, however isolate 6_13_25 had a similar trended towards reduced expression compared to TC-83. 
3 hours post infection

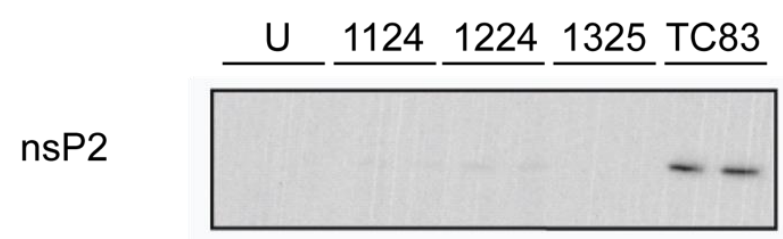

actin

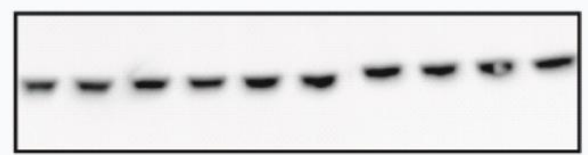

8 hours post infection

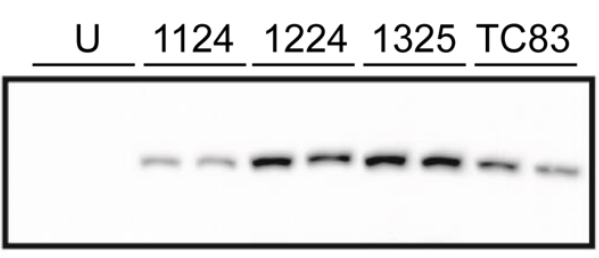

E protein

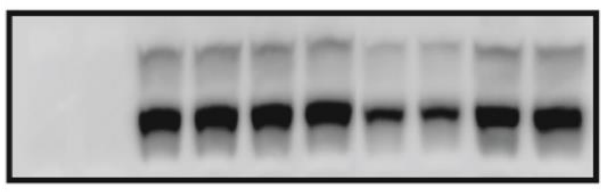

actin
5 hours post infection
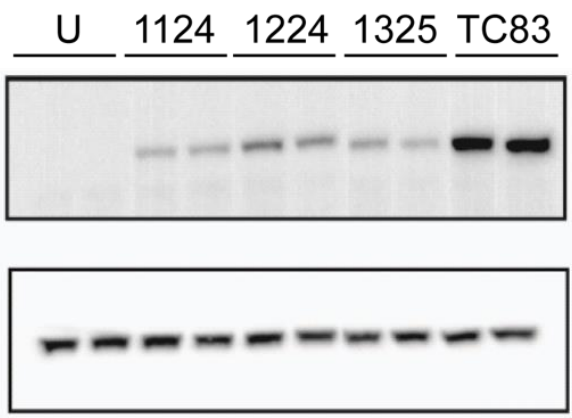

10 hours post infection
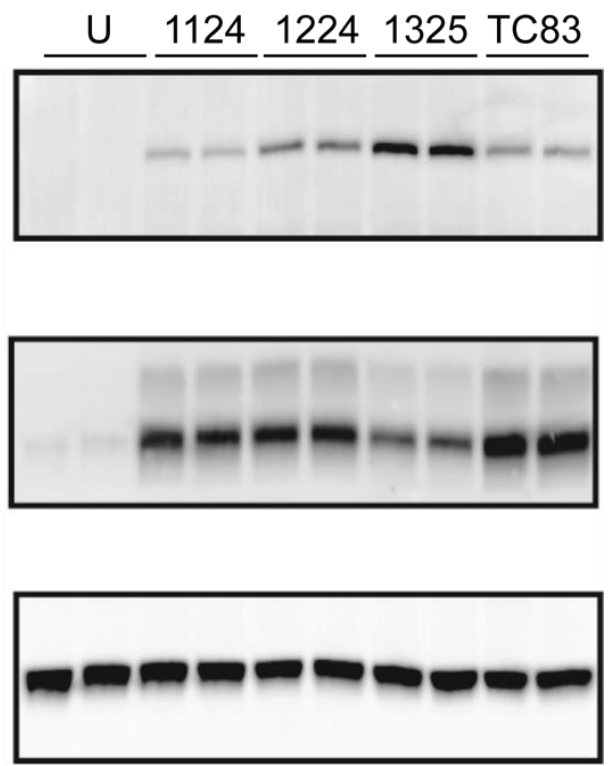

Figure 24. Representative Western blot images examining viral protein expression. Cell lysate was collected and used for Western blotting at the indicated times post infection. Lysate was probed for $\mathrm{nsP} 2$ and $\mathrm{E}$ protein as indicated, and actin was used was an internal loading control. Labels: U=uninfected, 1124=82_11_24, 1224=81_12_24, 1325=6_13_25. All of the mutant viruses display a delay in nsP2 production, similar to their delay in growth kinetics. However, only the double mutant, 1325 , showed a 
reduction in E protein synthesis. Representative images of one experiment of three. Each experiment had three biological replicates per virus and uninfected control. 
A.

3 HPI

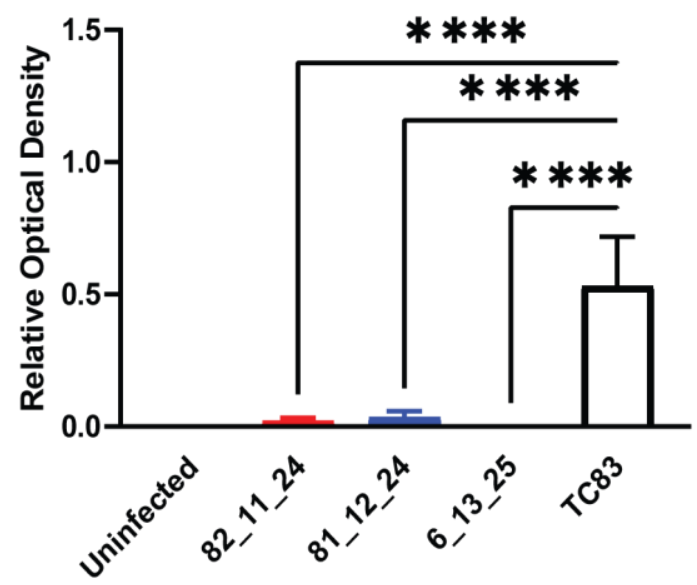

8 HPI

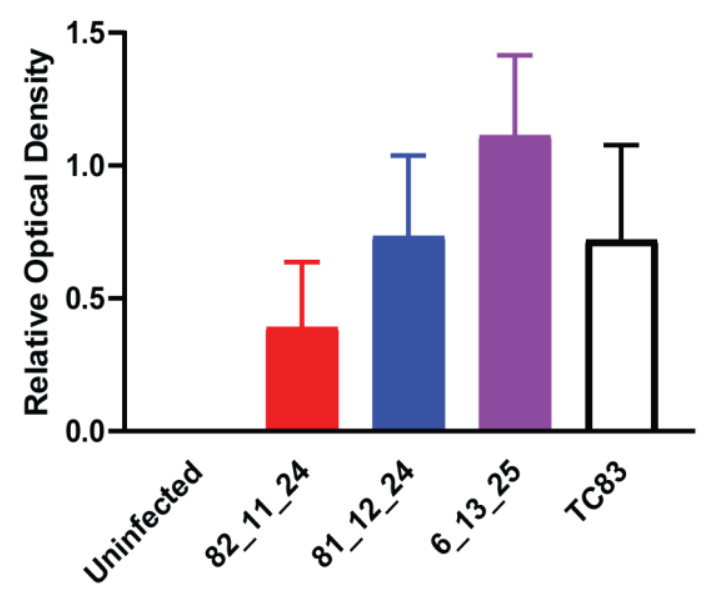

B.

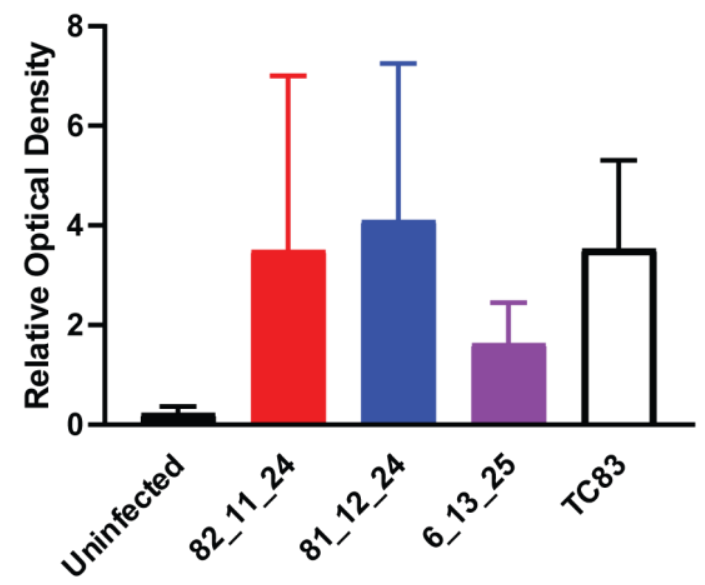

5 HPI

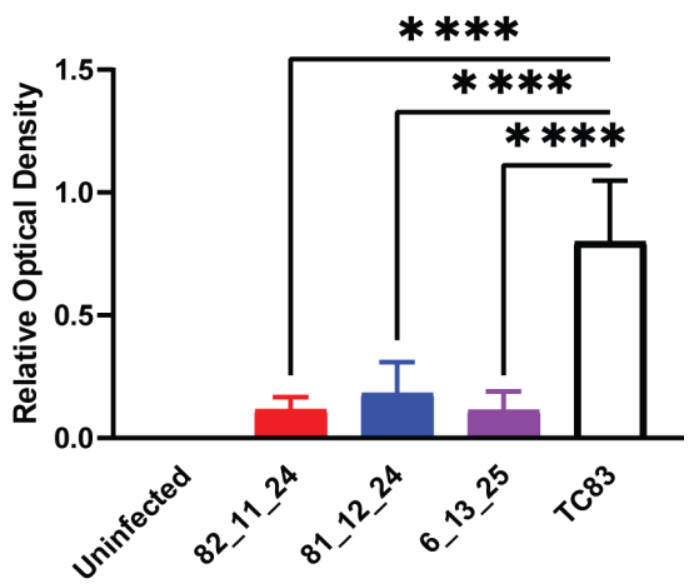

$10 \mathrm{HPI}$

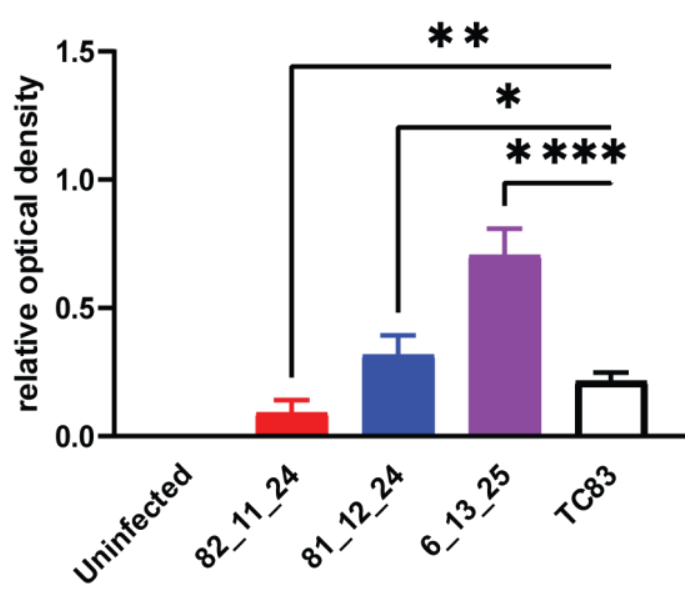

$10 \mathrm{HPI}$

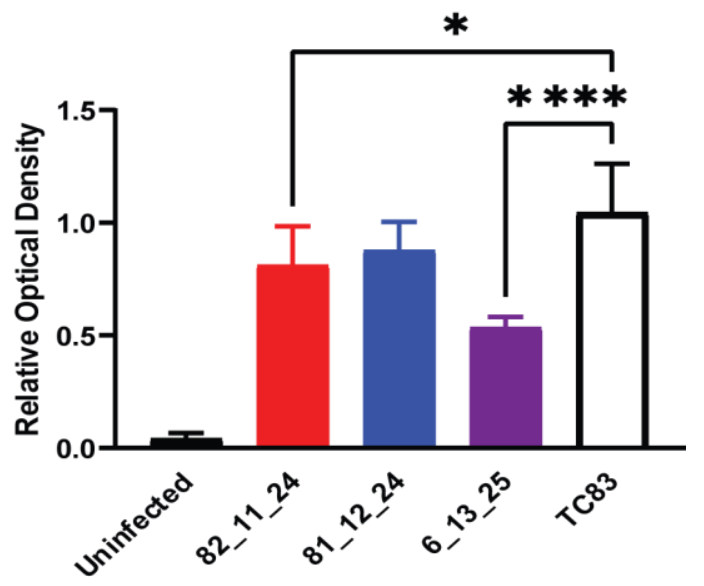


Figure 25. Quantification of viral protein expression. Using densitometry, the relative expression of nsP2 and the E protein was quantified. A) Quantification of nsP2 expression at the indicated times post infection. All mutant isolates show delayed expression, with less nsP2 present than in TC-83 at three and five hours post infection. By eight hours expression has caught up, and this is maintained at ten hours. B) Quantification of E protein expression. There is no significant difference between the viruses at eight hours post infection, however at ten hours post infection both isolate 6_13_25 and isolate 82_11_24 mutant show significant reductions in E protein expression compared to TC-83. Combined data from three experiments. Three biological replicates per group ${ }^{*} \mathrm{p}<0.05,{ }^{* *} \mathrm{p}<0.01,{ }^{* * *} \mathrm{p}<0.001,{ }^{* * * *} \mathrm{p}<0.0001$ as calculated using one way ANOVA. 


\section{Growth of VEEV TC83 and mutant isolates in a neuronal cell model}

While fibroblasts have historically been the cell of choice for much alphavirus work, these cells are only a model of the initial stages of infection. The severe pathology of VEEV, encephalitis and other neurological effects, primarily occurs in neurons and related cells and not in fibroblasts. Thus, it was decided to examine the mutant viral isolates for attenuation in a model of neuronal cells.

The SH-SY5Y cell line was selected for several reasons. When maintained in cell culture they have a neural progenitor cell like phenotype and can be maintained for several passages which is a significant advantage over other types of neural cell culture. These cells can also be differentiated into a neuron like phenotype if given the correct additives and media ${ }^{203}$. These neuron like cells have processes and are positive for markers of mature neurons ${ }^{205}$.

When growth kinetics were examined in undifferentiated SH-SY5Y cells, the delay in viral growth was reduced, Figure 26 A. While isolate 6_13_25 displayed a delay in growth, while still eventually reaching parity with TC-83, isolates 81_12_24 and

82_11_24 did not display significant differences from TC-83. This indicates that in these undifferentiated neuronal cells, the reduction in the structural genes or delay in subgenomic RNA synthesis is playing a significant role in the delay in viral growth, while the $\mathrm{N}$ terminal region of nsP2 appears to be less important. However, in the differentiated cells all of the mutant viral isolates had delayed growth and isolate 6_13_25 never reached parity with TC-83, ending the time course with a half log reduction in maximum titer, Figure $26 \mathrm{~B}$. This shows that in these differentiated cells, both the $\mathrm{N}$ terminal region of nsP2 and the effects of the subgenomic promoter region are important to normal virus growth. The normal synthesis of the subgenome and the structural proteins is also important to reaching normal titers in these cells. This indicates 
that the differences in physiology of the differentiated cells leads to different restrictions on viral growth. SH-SY5Y cells have been used as models for many different types of neural pathologies ${ }^{199,200,202,205,207}$, and these cells display a phenotype that is similar to mature neurons after differentiation. This results in many changes to the metabolism and biology of these cells that could restrict viral growth. This includes the production of various neuron specific biological markers as well as neurotransmitters, as well as a slowing and eventual stop of the cell cycle where these cells no longer divide. Any one of or combination of these changes may result in restricted and slow viral growth. For example, due to this slowing of the cells cycle there will be less resources present in the cell for the virus to use to fuel its own replication leading a slow in its replication. It is also possible that any of the many upregulated neural genes may also have off target effects that restrict viral replication. 
A.

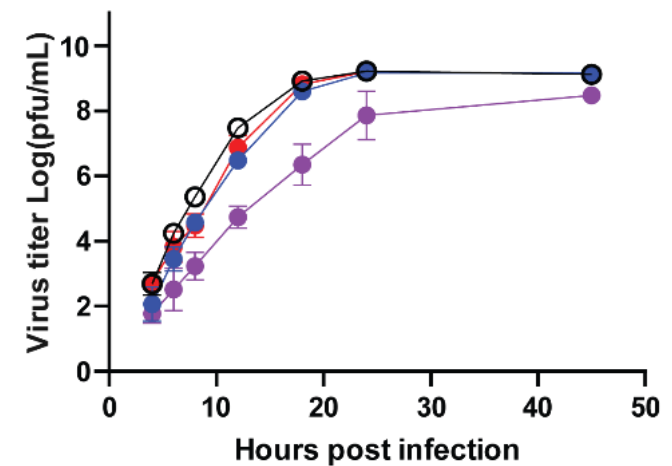

$0.05 \mathrm{MOI}$

B.

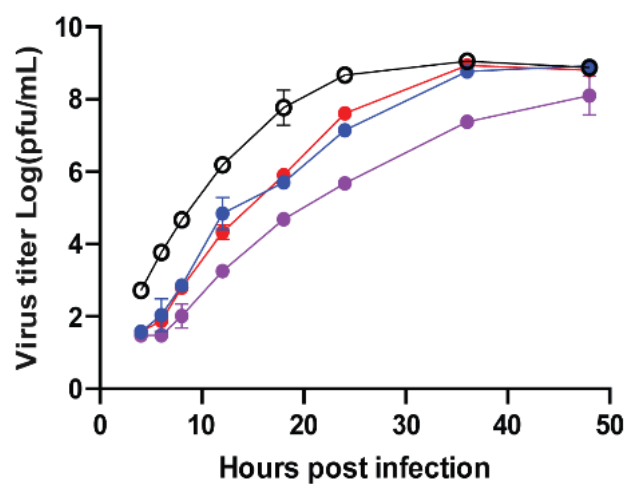

$5 \mathrm{MOI}$

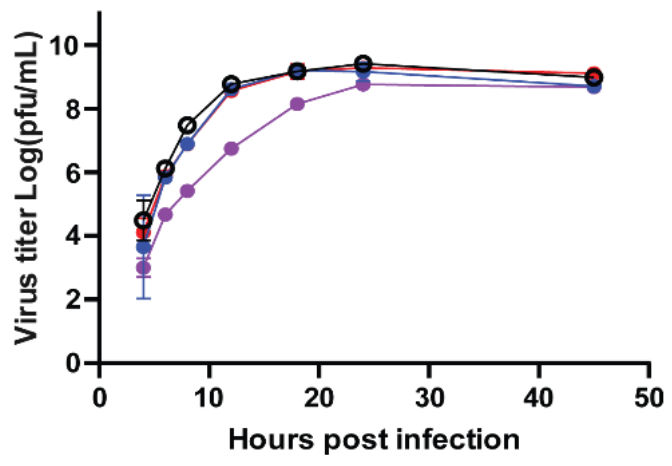

$5 \mathrm{MOI}$

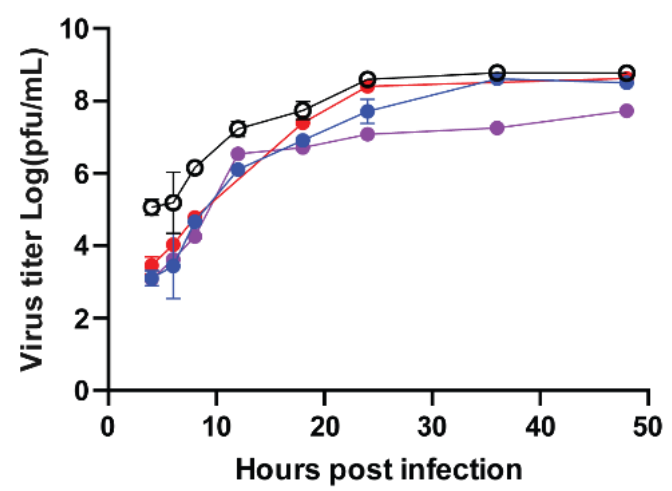

$$
\begin{aligned}
& \text { - TC-83 } \\
& --81 \_12 \_24 \\
& -6 \text { 8211124 } \\
& -6 \text { 6_13_25 }
\end{aligned}
$$

Figure 26. Compound resistant mutant viruses have delayed growth in a neural cell model. A) Undifferentiated SH-SY5Y cells were infected with the indicated viral strains at either 0.05 or $5 \mathrm{MOI}$. Supernatant was collected at the indicated time points and 
replication measured by plaque assay. In undifferentiated neural cells, there was little delay seen in the single mutants. However, growth of isolate 6_13_25 still showed a delay in growth. B) Differentiated SH-SY5Y cells were used in the same experiment as A. In this case all of the mutant isolates showed a delay in growth, and isolate 6_13_25 was generally attenuated as well, with a final titer fivefold less than the parental strain. Graphs are of individual experiments that were performed once. Three biological replicates per time point collected. 


\section{Discussion}

While alphaviruses are well studied, there remain portions of their genomes and proteins that lack described function and have remained difficult to characterize using typical methods of investigation. In this chapter viral isolates that resist treatment with antiviral compounds identified and derived in our lab were used as a method of viral biology characterization. Through the sequencing of these isolates, mutations were confirmed to be in an undescribed region of VEEV nsP2. This allowed for characterization of these mutants as a proxy for determining the function of the region.

Each of the isolated mutants had and additional mutation outside of the nsP encoding regions. Isolates 81_12_24 and 82_11_24 both had a single point mutation in their $\mathrm{E}$ proteins and isolate $6 \_13 \_25$ had a mutation in the subgenomic promoter region. While the mutations in the $\mathrm{E}$ proteins had no obvious effect in our characterization of these isolates, the subgenomic promoter mutations had significant effects. This mutation resulted in decreased subgenomic RNA synthesis structural gene expression. This mutation is likely involved in small plaque phenotype only seen in insolate 6_13_25, as well its increased attenuation in growth kinetics compared to the other isolates.

The region of nsP2 that was mutated appears to be important in normal RNA synthesis. As two of the isolates have delayed synthesis of positive-sense RNA. It is hypothesized that due to the location of these mutations in an external face of the protein, and far from the active helicase sites, that this region is likely involved in protein interactions that stabilize the nsP complex during replication. This delay in RNA synthesis also resulted in a delay in the expression of the viral nsPs, as indicated by reduced and delayed expression of nsP2. This delay would have cascading effects during infection. Due to its importance as a major driver of viral RNA synthesis, a slowing of nsP2 expression would delay RNA replication, which would result in a vicious 
cycle of reduced RNA leading to longer delays in protein synthesis. And this would, in turn, explain why these viruses have delayed replication. The fact that the viruses overcome this attenuation later in infection to reach similar maximal titers as TC-83 is explained by the exponential reduction of these molecules. Eventually so much nsP2 and RNA are created that the viral replication and production machinery, as well as the ribosomes, become wholly saturated, and further acceleration is not possible.

In addition to the work characterizing RNA replication, protein expression, and growth kinetics in fibroblast cells, a more restrictive neural cell line was used. SH-SY5Y cells are advantageous as a model because unlike other neuronal cell lines, they can be maintained for several passages in cell culture when kept in their immature state. This immature state is similar to neural progenitor cells. They can also be differentiated, commonly using retinoic acid, to mimic a mature neuron cell type.

There was no increase in attenuation when the mutant viral isolates were grown in the undifferentiated SH-SY5Y cells and in fact, they were more similar to TC-83 than in the fibroblasts. This is an interesting phenotype that deserves further study. It is possible that his has to with their state along the epithelial-mesenchymal axis, as these cells were derived from metastatic neuroblastoma that was extracted from bone marrow.

When these viral isolates were examined in differentiated SH-SY5Y cells, the attenuation was increased compared to TC-83. The growth delay was more significant and isolate 6_13_25 had a reduced maximum titer as well. This may be due to an increased basal activation level of antiviral signaling in the mature cells. However, as this is quite different from the undifferentiated cells, it is likely that this restriction is due to a change relating to the maturation of the neurons themselves. These cells have been found to behave in many ways like mature neurons, and it is possible that one of the many biochemical pathways that they activate results in the restriction of viral growth. 
This indicates that the $\mathrm{N}$ terminal region of $\mathrm{nsP2}$ is important for normal replication in these cells and should be investigated further for potential interactions with molecules that are expressed in these differentiated cells but not in undifferentiated SH-SY5Y cells.

\section{Conclusions}

From the work that has been presented here it is clear that mutations in the $\mathrm{N}$ terminal region of VEEV nsP2 are detrimental to production of new infectious virus, and that this results from a delay in RNA synthesis, leading to a delay in the production of the viral nonstructural proteins. While a detailed mechanism remains unclear, this region appears to be important to the RNA synthesis of the virus. The current hypothesis is that the mutations are disrupting the protein/protein interactions that help to form and stabilize the mature replicase complex, particularly between nsP2 and nsP4. This is due to these mutations being far from any sites known to be involved in the helicase or RNA binding activities of nsP2, and their localization to sites that are largely on external faces of the protein structure.

This $\mathrm{N}$ terminal region of $\mathrm{nsP2}$ is also important for replication in differentiated SH-SY5Y cells, but not in undifferentiated cells. This indicates that this region is likely to interaction with pathways or components of the mature cells that are nor expressed in the neuroprogenitor like cells. This should be further investigated, looking for potential interaction partners in the cell, as well for pathways that are altered in these cells upon infection. 
CHAPTER 5

DISUCSSION AND FUTURE DIRECTIONS 


\section{DISCUSSION}

This work has investigated the mechanism of action and interactions of a novel antiviral amidine scaffold with Venezuelan equine encephalitis virus (VEEV). These compounds were also used to generate mutant virus populations that were used to investigate heretofore unknown aspects of VEEV biology. This work has shown how these compounds have promise both as potential therapeutics for VEEV induced disease, as well as for probing biological activities of uncharacterized portions of the viral genome. In this manner, these compounds have future usefulness both as potential treatments, as well as in the development of further compounds targeting novel biological activities that they can be used to detect and characterize.

The compound scaffold was characterized through the methods described in chapter two. It was found that these compounds specifically and efficiently inhibit the synthesis of VEEV RNA during infection, and that this activity is maintained in a cell free system. The specificity of these compounds is highly desirable, as it means there are less likely to be off target effects when they are moved to more complex systems such as animal models. The RNA synthesis inhibitory effect of these compounds is also novel in the development of anti-VEEV molecules, as there are no other direct RNA synthesis inhibitors under investigation outside of our research group and collaborators. This is contrasted to nucleoside analogs, which are popular drug targets, but only inhibit viral RNA synthesis indirectly.

This work characterizing the antiviral activity of these compounds led to our hypothesis that there is a direct interaction between the compounds and the viral proteins. This is due to the fact that compound ML336 maintains its effectiveness in a cell-free RNA synthesis assay. While this assay is not able to fully confirm an interaction between the viral proteins and compounds due to the inclusion of some cellular 
components, its minimal nature makes it more likely that the compounds are directly interfering with the viral proteins. This led to investigation described in chapter 3 , attempting to identify and characterize this proposed interaction between the amidine compounds and the VEEV nsPs.

Unfortunately, it was not possible to confirm or deny the existence of this interaction, as the data obtained from the experiments looking to detect this binding were largely inconclusive. However, there were several advances that were made through this work. First, a reliable method of expressing the $\mathrm{N}$ terminal domain of VEEV nsP2 was developed. While this protein was not able to be used for the full range of characterization studies desired, this system has been well optimized for expression, and large parts of its purification. Adding a useful tool for future binding kinetics assays, as well as biochemical experiments examining the effects of these compounds on the enzymatic activities of the $\mathrm{N}$ terminal region of nsP2.

Additionally, a technique using fluorescent microscopy with a double stranded RNA antibody as a readout for active viral replication was performed for the first time in our lab. This tool allows for the detection of both localization of the replication activity of this virus in infected cells, and quantification of how many cells are undergoing active viral production at any given time during infection, as opposed to cells that may no longer contain actively replicating virus, but still express viral proteins. For this same assay, click chemistry compatible compounds were developed by our medicinal chemist collaborator, opening up many new avenues for compound manipulation. The modified functional groups of these compounds allow for a variety of detection methods, as described here, as well as purification of the compounds from complex mixtures, as has been previously performed with labeled proteins ${ }^{215}$, depending on the desired use. 
In addition to being useful for therapeutic development, antiviral compounds can also be used as chemical probes of viral biology. These compounds can be used to generate resistant mutant viral isolates, and these mutations can then be mapped to the viral genome, indicating the region(s) that are important in the activity of the compounds. This is useful from both a drug mechanism standpoint as well as a viral biology standpoint. For example, in this work it has been shown that the amidine antiviral scaffold functions to inhibit viral RNA synthesis. This means that any mutations that occur in the viral genome which confer resistance to drug treatment are in areas that are likely to be highly important for the synthesis of viral RNA. When these experiments were performed using the amidine scaffold compounds, it was found was that the resistance mutations consistently mapped to the $\mathrm{N}$ terminal domain of VEEV nsP2, a region of currently undescribed function.

The localization of mutations to this region is not altogether surprising, as nsP2 is highly important in viral RNA synthesis, acting as the viral RNA helicase. NsP2 is also responsible for the normal cleavage of the polyprotein, which regulates RNA synthesis during viral infection ${ }^{216-219}$. As the implicated region lacked a specific function it was hypothesized that it was involved the normal synthesis of viral RNA. Due the location of the mutations on an external face of the protein, as well as the mutations being found primarily in less ordered regions, this activity was predicted to be mediated by interfering with the protein/protein interactions of the replicase complex and destabilizing these interactions.

A group of mutant isolates was selected and submitted for further characterization studies. First, it is important to point out that these mutations were not found in detectable levels in unselected viral populations, indicating that these mutations are likely to be detrimental to the virus in the absence of selection by the compound. 
Initially these viral isolates were examined in a growth curve using highly permissive fibroblast cells, this experiment showed that the compound resistant isolates had delayed growth compared to the parental VEEV strain used, but they did reach the same peak titers late in infection. This result was then mirrored in their RNA synthesis profiles with the mutants having delayed synthesis of both positive and negative sense genomic RNA.

Three mutant viruses were selected for further characterization. Two isolates contained only single point mutations in $\mathrm{nsP} 2$ as well as a single point mutation in their $\mathrm{E}$ proteins. These isolates were predicted to be attenuated and display alterations in their RNA synthesis. A third isolate had a double mutation in nsP2 as well as a point mutation in its subgenomic promoter region. This isolated was predicted to be highly attenuated due to expected changes in the synthesis of its subgenomic RNA, and it was unclear how the mutations in nsP2 would combine with this to effect RNA synthesis and viral replication.

Each of these viruses was attenuated in fibroblast cells, having delayed growth compared to the parental strain. As predicted the isolate containing the mutation in the subgenomic promoter was strongly attenuated, with the other two isolates having intermediate phenotype. However, it was surprising that each these isolates eventually reached parity with the parental strain and had no reduction in maximum titer. Similar phenotypes were seen when RNA synthesis was examined, and as predicted, the isolate with the subgenomic promoter mutation had a decrease in subgenomic RNA synthesis that the other two isolates did not display. The effects of these mutations on protein expression were slightly different, each of the isolates had a significant delay in the production of the viral nsPs and did not reach parental expression levels until eight 
hours post infection. As expected, only the subgenomic promoter mutation had an effect on the synthesis of the structural proteins.

When combined this data indicates that mutations in the $\mathrm{N}$ terminal region of nsP2 lead to delays in RNA synthesis leading to delays in nsP expression. As the nsPs are responsible for the replication of the viral RNA, these effects compound during infection, slowing viral replication, and leading to the delayed growth that was seen. However, as there was no loss in titer, and expression levels of both RNA and protein reach parity with TC-83 later in infection, these effects are overcome. This is most likely due to the nature of the exponential production of viral materials during infection. Even though the log phase growth of the virus is delayed, the viruses are still able to produce enough of their components over time to saturate their replicative systems as well as those of the cell, at which point no more acceleration of replication is possible. This results in the maximum titer being achieved, even though it takes longer to reach. A model outlining this hypothesis can be found in Figure 27.

These phenotypes had all been examined in a highly permissive fibroblast cell line. These cells lack the ability to activate their interferon system and so are unable to enter an antiviral state. It was hypothesized that the mutant viral isolates would be increasingly attenuated in a cell system that could activate the antiviral response, so a second cell line was selected to examine this. SH-SY5Y cells were selected. These cells have a neural progenitor cell like phenotype and can also be differentiated into a mature neuron like state, making them an excellent model of VEEV infection in neurons, a significant site of pathology in the host $220,221$.

The growth kinetics of the mutant viral isolates were examined in both the differentiated and undifferentiated cells. In the immature cells, the growth delay was less significant than that seen in the fibroblast cells. Only the isolate containing the 
subgenomic promoter mutation was found to have a significant delay compared to TC83 and as before it still reached parity alter in infection. However, in the differentiated cells each of the isolates was significantly attenuated, and the isolates with the subgenomic promoter mutation had a final titer reduction of fivefold. This difference 
A.

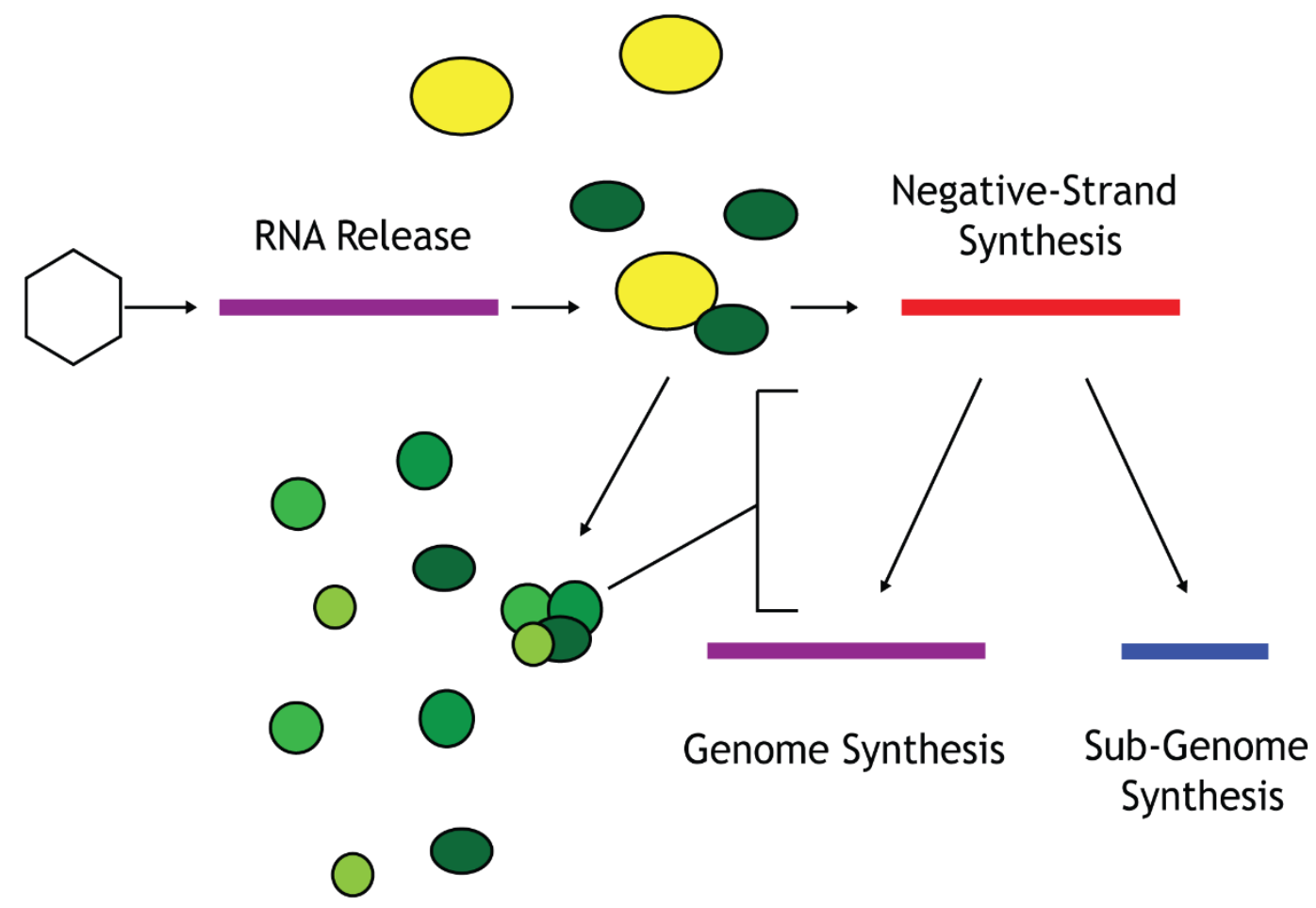

B.

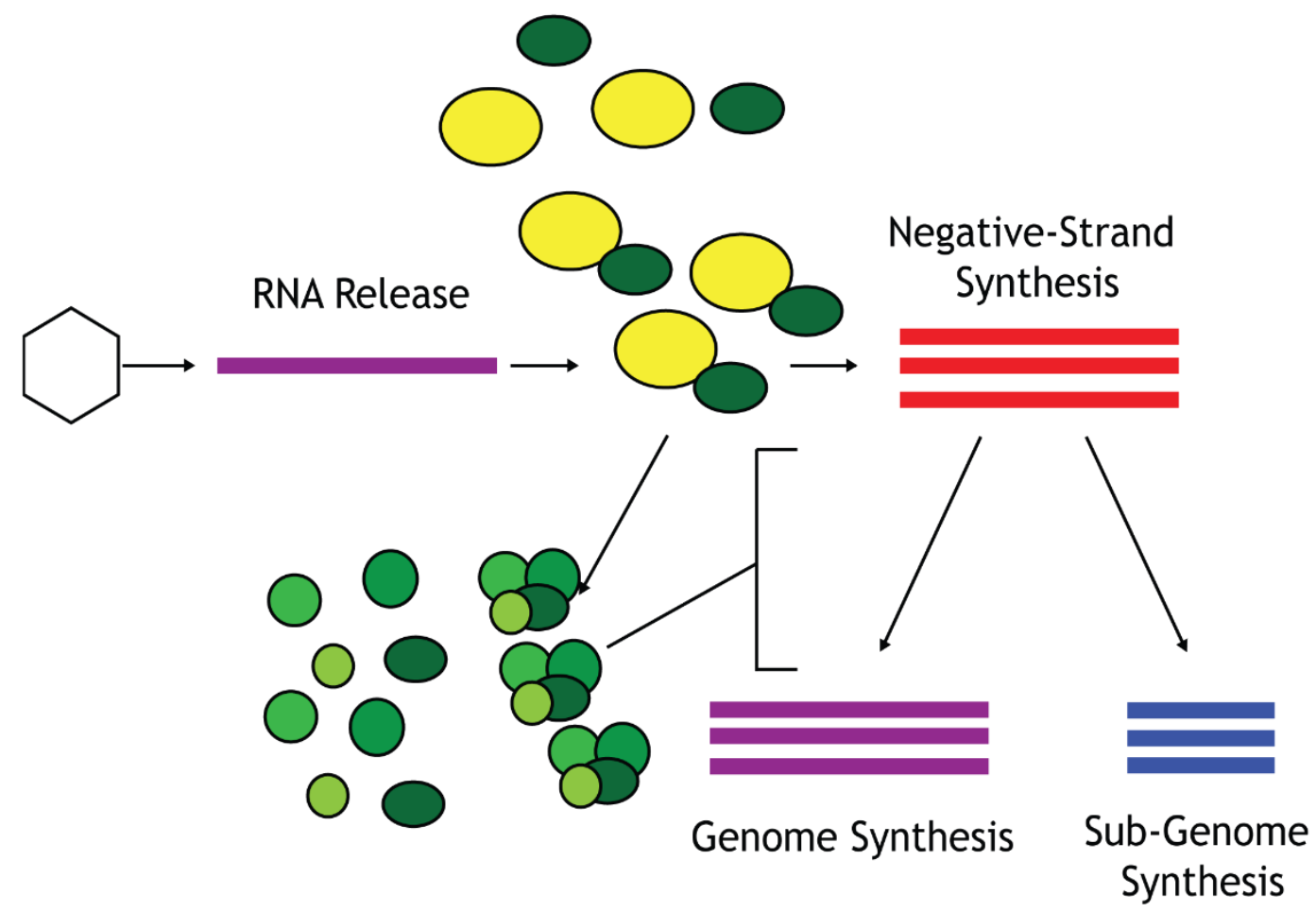


Figure 27. Working model of the effects of mutations in the $\mathrm{N}$-terminal region of VEEV nsP2. A) Early in infection the nonstructural polyprotein is synthesized from the genomic RNA. However, due the presence of mutations, the protein interactions have reduced stability, resulting in a significant reduction in the number of active complexes. This reduces the rate of viral RNA synthesis. B) As infection proceeds to later time points, the amount of nsPs synthesized increases. This increases the number of complexes that form despite the reduction in interaction stability. This leads to an increase in the rate of viral RNA synthesis, eventually reaching the maximum rate seen in infection with the parental TC-83 strain. 
between the differentiated and undifferentiated cells implies that the $\mathrm{N}$ terminal region of nsP2 is likely important to the normal replication of VEEV in mature neurons but not in other neural derived cells, such a neural progenitor cells. The differentiated cells go through significant physiological changes during the maturation process leading to a host of phenotypes that make them useful models for neurological disease $200,202,207,221$. This includes the production of neurotransmitter, the formation of neural processes, and loss of cell cycling behavior. Any one or multiple of these changes could lead to more restricted replication of VEEV. Of particular interest is the way that the senescence of these cells may restrict the amount of resources available for viral replication, which is likely to perturb the ability of the virus to replicate as quickly as it would in the dividing undifferentiated cells.

To summarize the work presented in this dissertation, the amidine scaffold compounds developed by our lab for the treatment of VEEV are highly effective and specific at inhibiting VEEV RNA synthesis. This activity is maintained in a cell-free system and has no effect on cellular RNA. These compounds are currently being used as the foundation for pretherapeutic drug discovery and development with collaborators. These compounds are predicted to interact with the nsPs of VEEV. While this interaction remains unconfirmed, several useful biochemical assays were developed, and can be used for further characterization of both antiviral compounds and viruses. A system was also developed for the recombinant expression of the $\mathrm{N}$ terminal region of $\mathrm{nsP} 2$, and this system is ready for use in biochemical and pharmacological assays. The amidine compounds were also used to derive resistant viral isolates that were used to characterize the $\mathrm{N}$ terminal region of VEEV vsP2. It was found that this region is important to the synthesis of viral RNA, and that its perturbation leads to a delay in viral 
growth. This region is also important in the infection of differentiated neurons, but not undifferentiated neuronal cells. The mechanism of this phenotype remains unknown.

\section{FUTURE DIRECTIONS}

The work presented here has left several unanswered questions that need to be further examined and opened new questions.

First, it remains unclear how and if the amidine scaffold compounds are interacting with the proteins of VEEV. While the sequencing results from chapter 4 clearly indicate that mutations occur in a predictable manner to escape compound treatment, it has yet to be shown that the compounds and viral proteins directly interact. In chapter 3 several methods that were used to attempt to describe this interaction were summarized. However, no conclusive results were obtained. The primary way that this work should be continued is continuing to pursue the use of ectopically expressed protein for use in biochemical and pharmacological assays.

The production of the $\mathrm{N}$ terminal region of $\mathrm{nsP2}$ in a soluble form has been well optimized as has its initial purification. Further work remains in optimizing the cleavage of the SUMO tag from the construct, which will be necessary for any functional assays to be performed, such as helicase activity assays. The currently expressible protein is already usable in many types of binding assays such a microscale thermophoresis or surface plasmon resonance. While each of these assays requires highly pure sample, tag removal is not necessary so the current construct can be used. Pilot studies using SPR have been performed, however sensitivity was poor and further optimization of this assay is required. 
Secondly, the work with the resistant viral isolates has shown that as expected, the $\mathrm{N}$ terminal region of nsp2 appears to be important to viral RNA synthesis. However, each of the isolates tested had an additional mutation. The $\mathrm{N}$ terminal region needs to be isolated to confirm that these phenotypes are due to the mutations in this region. To do this, a reverse genetic system can used. Plasmid based clones of TC-83 are available, and the desired mutations can be inserted using site directed mutagenesis. With the use of in vitro transcription these plasmids can be transfected into cells and virus generated with the desired genotypes. These viruses can then be submitted to the same experimenters as already performed with the mutant viral isolates. This type of system can also be used to isolate the double nsP2 mutation seen in one isolate, which was confounded by the presence of a mutation in its subgenomic promoter.

Finally, the work with the resistant viral isolates in neuronal cells indicated that the $\mathrm{N}$ terminal region of VEEV nsP2 is important for the normal infection of differentiated neurons. The effect of mutating this region was more significant in these cells than an either fibroblasts or undifferentiated neuronal cells. This lead to the hypothesis that a feature unique to the differentiated neural cells was involved in this enhanced restriction on viral replication. This should first be investigated by examining the differential gene expression of the differentiated and undifferentiated cells to determine their differences in gene expression that may explain this alteration in sensitivity to viral infection. Further transcriptional changes in response to infection can then be determined by performing single cell sequencing and isolating those genes that upregulated in the infected but not uninfected cells, to characterize the response to infection. Ideally, there will be known antiviral signals or vial interactions that can be pursued in further, more detailed experiments. If not, there are a variety of ways that protein interactions can be predicted to lead to potential genes of interest. 


\section{REFERENCES}

1. INternation Committee on Taxonomy of Viruses. Taxonomy. (2020).

2. Powers, A. M. et al. Evolutionary Relationships and Systematics of the Alphaviruses. J. Virol. 75, 10118-10131 (2001).

3. Crosby, L. et al. Severe manifestations of chikungunya virus in critically ill patients during the 2013-2014 Caribbean outbreak. Int. J. Infect. Dis. 48, 78-80 (2016).

4. Rezza, G. et al. Infection with chikungunya virus in Italy: an outbreak in a temperate region. The Lancet 370, 1840-1846 (2007).

5. Grandadam, M. et al. Chikungunya Virus, Southeastern France. Emerg. Infect. Dis. 17, 910913 (2011).

6. Staples, J. E. \& Fischer, M. Chikungunya Virus in the Americas - What a Vectorborne Pathogen Can Do. N. Engl. J. Med. 371, 887-889 (2014).

7. Robert E. Johnston, C. J. P. Fields Virology. vol. 1 (Lippincott-Raven, 2018).

8. Griffin, D. E. \& Johnson, R. T. Role of the Immune Response in Recovery from Sindbis Virus Encephalitis in Mice. 7.

9. Laine, M. et al. Prolonged arthritis associated with Sindbis-related (Pogosta) virus infection. Rheumatology 39, 1272-1274 (2000).

10. Adouchief, S., Smura, T., Sane, J., Vapalahti, O. \& Kurkela, S. Sindbis virus as a human pathogen-epidemiology, clinical picture and pathogenesis. Rev. Med. Virol. 26, 221-241 (2016).

11. Go, Y. Y., Balasuriya, U. B. R. \& Lee, C. Zoonotic encephalitides caused by arboviruses: transmission and epidemiology of alphaviruses and flaviviruses. Clin. Exp. Vaccine Res. 3, 58-77 (2014).

12. Acosta-Ampudia, Y. et al. Mayaro: an emerging viral threat? Emerg. Microbes Infect. 7, (2018).

13. Murillo-Zamora, E. et al. Persistent Arthralgia and Related Risks Factors: A Cohort Study at 12 Months from Laboratory-Confirmed Chikungunya Infection. Arch. Med. Res. 49, 65-73 (2018).

14. Practitioners, T. R. A. C. of G. RACGP - Ross River virus. https://www.racgp.org.au/afp/2009/august/ross-river-virus/.

15. Aguilar, P. V. et al. Endemic Venezuelan equine encephalitis in the Americas: hidden under the dengue umbrella. Future Virol. 6, 721-740 (2011).

16. Calisher, C. H. Medically important arboviruses of the United States and Canada. Clin. Microbiol. Rev. 7, 89-116 (1994).

17. Deresiewicz, R. L., Thaler, S. J., Hsu, L. \& Zamani, A. A. Clinical and Neuroradiographic Manifestations of Eastern Equine Encephalitis. http://dx.doi.org/10.1056/NEJM199706263362604 https://www.nejm.org/doi/10.1056/NEJM199706263362604?url_ver=Z39.882003\&rfr_id=ori\%3Arid\%3Acrossref.org\&rfr_dat=cr_pub\%3Dwww.ncbi.nlm.nih.gov (2009) doi:10.1056/NEJM199706263362604.

18. Statistics \& Maps | Eastern Equine Encephalitis | CDC. https://www.cdc.gov/easternequineencephalitis/tech/epi.html (2019).

19. Simon, L. V., Coffey, R. \& Fischer, M. A. Western Equine Encephalitis. in StatPearls (StatPearls Publishing, 2021). 
20. Bergren, N. A. et al. "Submergence" of Western equine encephalitis virus: Evidence of positive selection argues against genetic drift and fitness reductions. PLOS Pathog. 16, e1008102 (2020).

21. Rusnak, J. M., Dupuy, L. C., Niemuth, N. A., Glenn, A. M. \& Ward, L. A. Comparison of Aerosol- and Percutaneous-acquired Venezuelan Equine Encephalitis in Humans and Nonhuman Primates for Suitability in Predicting Clinical Efficacy under the Animal Rule. Comp. Med. 68, 380-395 (2018).

22. Federal Select Agent Program - Select Agents and Toxins List. https://www.selectagents.gov/SelectAgentsandToxinsList.html.

23. Steele, K. E. \& Twenhafel, N. A. REVIEW PAPER: Pathology of Animal Models of Alphavirus Encephalitis. Vet. Pathol. 47, 790-805 (2010).

24. Carrara, A.-S. et al. Venezuelan Equine Encephalitis Virus Infection of Cotton Rats. Emerg. Infect. Dis. 13, 1158-1165 (2007).

25. S. C. Weaver et al. Genetic determinants of Venezuelan equineencephalitis emergence. Arch. Virol. 43-64 (2004).

26. Zacks, M. A. \& Paessler, S. ENCEPHALITIC ALPHAVIRUSES. Vet. Microbiol. 140, 281 (2010).

27. Howard, J. J., Morris, C. D., Emord, D. E. \& Grayson, M. A. Epizootiology of Eastern Equine Encephalitis Virus in Upstate New York, Usa. Vii. Virus Surveillance 1978-85, Description of 1983 Outbreak, and Series Conclusions. J. Med. Entomol. 25, 501-514 (1988).

28. Armstrong, P. M. \& Andreadis, T. G. Eastern Equine Encephalitis Virus in Mosquitoes and Their Role as Bridge Vectors. Emerg. Infect. Dis. 16, 1869-1874 (2010).

29. Wayne J. Crans. The Status of Aedes Solicitans as an Epidemic Vector of Estern Equine Encephalitis in New Jersey. Mosq. News 37, 85-85 (1977).

30. Smith, D. R., Adams, A. P., Kenney, J. L., Wang, E. \& Weaver, S. C. Venezuelan equine encephalitis virus in the mosquito vector Aedes taeniorhynchus: Infection initiated by a small number of susceptible epithelial cells and a population bottleneck. Virology $\mathbf{3 7 2}$, 176-186 (2008).

31. Forrester, N. L., Coffey, L. L. \& Weaver, S. C. Arboviral Bottlenecks and Challenges to Maintaining Diversity and Fitness during Mosquito Transmission. Viruses 6, 3991-4004 (2014).

32. Arrigo, N. C., Smith, D. R., Weaver, S. C., Muehlberger, L. E. \& Leal, G. Infection and Dissemination of Venezuelan Equine Encephalitis Virus in the Epidemic Mosquito Vector, Aedes taeniorhynchus. Am. J. Trop. Med. Hyg. 77, 176-187 (2007).

33. Leung, J. Y.-S., Ng, M. M.-L. \& Chu, J. J. H. Replication of Alphaviruses: A Review on the Entry Process of Alphaviruses into Cells. Adv. Virol. 2011, 1-9 (2011).

34. MacDonald, G. H. \& Johnston, R. E. Role of dendritic cell targeting in Venezuelan equine encephalitis virus pathogenesis. J. Virol. 74, 914-922 (2000).

35. Smith, A. L. \& Tignor, G. H. Host cell receptors for two strains of Sindbis virus. Arch. Virol. 66, 11-26 (1980).

36. Zhang, R. et al. Mxra8 is a receptor for multiple arthritogenic alphaviruses. Nature 557, 570-574 (2018).

37. Ma, H. et al. LDLRAD3 is a receptor for Venezuelan equine encephalitis virus. Nature 588, 308-314 (2020).

38. Bernard, K. A., Klimstra, W. B. \& Johnston, R. E. Mutations in the E2 glycoprotein of Venezuelan equine encephalitis virus confer heparan sulfate interaction, low morbidity, and rapid clearance from blood of mice. Virology 276, 93-103 (2000). 
39. Heil, M. L., Albee, A., Strauss, J. H. \& Kuhn, R. J. An Amino Acid Substitution in the Coding Region of the E2 Glycoprotein Adapts Ross River Virus To Utilize Heparan Sulfate as an Attachment Moiety. J. Virol. 75, 6303-6309 (2001).

40. Klimstra, W. B., Ryman, K. D. \& Johnston, R. E. Adaptation of Sindbis Virus to BHK Cells Selects for Use of Heparan Sulfate as an Attachment Receptor. J. Virol. 72, 7357-7366 (1998).

41. Helenius, A., Kartenbeck, J., Simons, K. \& Fries, E. On the entry of semliki forest virus into BHK-21 cells. J. Cell Biol. 84, 404-420 (1980).

42. Kolokoltsov, A. A., Fleming, E. H. \& Davey, R. A. Venezuelan equine encephalitis virus entry mechanism requires late endosome formation and resists cell membrane cholesterol depletion. Virology 347, 333-342 (2006).

43. Hernandez, R., Luo, T. \& Brown, D. T. Exposure to low $\mathrm{pH}$ is not required for penetration of mosquito cells by Sindbis virus. J. Virol. 75, 2010-2013 (2001).

44. Paredes, A. M. et al. Conformational changes in Sindbis virions resulting from exposure to low $\mathrm{pH}$ and interactions with cells suggest that cell penetration may occur at the cell surface in the absence of membrane fusion. Virology 324, 373-386 (2004).

45. Bernard, E. et al. Endocytosis of Chikungunya Virus into Mammalian Cells: Role of Clathrin and Early Endosomal Compartments. PLOS ONE 5, e11479 (2010).

46. Omar, A. \& Koblet, H. Semliki Forest virus particles containing only the E1 envelope glycoprotein are infectious and can induce cell-cell fusion. Virology 166, 17-23 (1988).

47. Sanz, M. A., Rejas, M. T. \& Carrasco, L. Individual Expression of Sindbis Virus Glycoproteins. E1 Alone Promotes Cell Fusion. Virology 305, 463-472 (2003).

48. Wahlberg, J. M., Boere, W. A. \& Garoff, H. The heterodimeric association between the membrane proteins of Semliki Forest virus changes its sensitivity to low $\mathrm{pH}$ during virus maturation. J. Virol. 63, 4991-4997 (1989).

49. Wengler, G. \& Wengler, G. In vitro analysis of factors involved in the disassembly of Sindbis virus cores by $60 \mathrm{~S}$ ribosomal subunits identifies a possible role of low pH. J. Gen. Virol. 83, 2417-2426 (2002).

50. Schlegel, A., Omar, A., Jentsch, P., Morell, A. \& Kempf, C. Semliki Forest virus envelope proteins function as proton channels. Biosci. Rep. 11, 243-255 (1991).

51. Spyr, C. A., Käsermann, F. \& Kempf, C. Identification of the pore forming element of Semliki Forest virus spikes. FEBS Lett. 375, 134-136 (1995).

52. Wengler, G., Koschinski, A., Wengler, G. \& Dreyer, F. Entry of alphaviruses at the plasma membrane converts the viral surface proteins into an ion-permeable pore that can be detected by electrophysiological analyses of whole-cell membrane currents. J. Gen. Virol. 84, 173-181 (2003).

53. Singh, I. \& Helenius, A. Role of ribosomes in Semliki Forest virus nucleocapsid uncoating. J. Virol. 66, 7049-7058 (1992).

54. Wengler, G., Würkner, D. \& Wengler, G. Identification of a sequence element in the alphavirus core protein which mediates interaction of cores with ribosomes and the disassembly of cores. Virology 191, 880-888 (1992).

55. Strauss, J. H. \& Strauss, E. G. The Alphaviruses: Gene Expression, Replication, and Evolution. MICROBIOL REV 72.

56. Strauss, E. G., Rice, C. M. \& Strauss, J. H. Sequence coding for the alphavirus nonstructural proteins is interrupted by an opal termination codon. Proc. Natl. Acad. Sci. 80, 5271-5275 (1983). 
57. Myles, K. M., Kelly, C. L. H., Ledermann, J. P. \& Powers, A. M. Effects of an Opal Termination Codon Preceding the nsP4 Gene Sequence in the O'Nyong-Nyong Virus Genome on Anopheles gambiae Infectivity. J. Virol. 80, 4992-4997 (2006).

58. Vasiljeva, L., Valmu, L., Kääriäinen, L. \& Merits, A. Site-specific protease activity of the carboxyl-terminal domain of Semliki Forest virus replicase protein nsP2. J. Biol. Chem. 276, 30786-30793 (2001).

59. Vasiljeva, L. et al. Regulation of the sequential processing of Semliki Forest virus replicase polyprotein. J. Biol. Chem. 278, 41636-41645 (2003).

60. Lemm, J. A. \& Rice, C. M. Roles of nonstructural polyproteins and cleavage products in regulating Sindbis virus RNA replication and transcription. J. Virol. 67, 1916-1926 (1993).

61. Shirako, Y. \& Strauss, J. H. Regulation of Sindbis virus RNA replication: uncleaved P123 and nsP4 function in minus-strand RNA synthesis, whereas cleaved products from P123 are required for efficient plus-strand RNA synthesis. J. Virol. 68, 1874-1885 (1994).

62. Lulla, A., Lulla, V. \& Merits, A. Macromolecular assembly-driven processing of the $2 / 3$ cleavage site in the alphavirus replicase polyprotein. J. Virol. 86, 553-565 (2012).

63. Lulla, V. et al. Timeliness of Proteolytic Events Is Prerequisite for Efficient Functioning of the Alphaviral Replicase. J. Virol. 92, (2018).

64. Liu, X. et al. Decreased Virulence of Ross River Virus Harboring a Mutation in the First Cleavage Site of Nonstructural Polyprotein Is Caused by a Novel Mechanism Leading to Increased Production of Interferon-Inducing RNAs. mBio 9, (2018).

65. Shin, G. et al. Structural and functional insights into alphavirus polyprotein processing and pathogenesis. Proc. Natl. Acad. Sci. 109, 16534-16539 (2012).

66. Skidmore, A. M., Adcock, R. S., Jonsson, C. B., Golden, J. E. \& Chung, D.-H. Benzamidine ML336 inhibits plus and minus strand RNA synthesis of Venezuelan equine encephalitis virus without affecting host RNA production. Antiviral Res. 174, 104674 (2020).

67. Pietilä, M. K., Hellström, K. \& Ahola, T. Alphavirus polymerase and RNA replication. Virus Res. 234, 44-57 (2017).

68. LaPointe, A. T., Moreno-Contreras, J. \& Sokoloski, K. J. Increasing the Capping Efficiency of the Sindbis Virus nsP1 Protein Negatively Affects Viral Infection. mBio 9, (2018).

69. Paul, D. \& Bartenschlager, R. Architecture and biogenesis of plus-strand RNA virus replication factories. World J. Virol. 2, 32-48 (2013).

70. Salonen, A., Ahola, T. \& Kääriäinen, L. Viral RNA Replication in Association with Cellular Membranes. Membr. Traffick. Viral Replication 285, 139-173 (2005).

71. Johan A. den Boon \& Ahlquist, P. Organelle-Like Membrane Compartmentalization of Positive-Strand RNA Virus Replication Factories. Annu. Rev. Microbiol. 64, 241-256 (2010).

72. Kopek, B. G., Perkins, G., Miller, D. J., Ellisman, M. H. \& Ahlquist, P. Three-Dimensional Analysis of a Viral RNA Replication Complex Reveals a Virus-Induced Mini-Organelle. PLoS Biol. 5, (2007).

73. Ertel, K. J. et al. Cryo-electron tomography reveals novel features of a viral RNA replication compartment. elife 6,.

74. Pietilä, M. K., van Hemert, M. J. \& Ahola, T. Purification of Highly Active Alphavirus Replication Complexes Demonstrates Altered Fractionation of Multiple Cellular Membranes. J. Virol. 92, (2018).

75. Hellström, K. et al. Partially Uncleaved Alphavirus Replicase Forms Spherule Structures in the Presence and Absence of RNA Template. J. Virol. 91, (2017).

76. Kallio, K. et al. Template RNA Length Determines the Size of Replication Complex Spherules for Semliki Forest Virus. J. Virol. 87, 9125-9134 (2013). 
77. Frolova, E. I., Gorchakov, R., Pereboeva, L., Atasheva, S. \& Frolov, I. Functional Sindbis Virus Replicative Complexes Are Formed at the Plasma Membrane. J. Virol. 84, 1167911695 (2010).

78. Thaa, B. et al. Differential Phosphatidylinositol-3-Kinase-Akt-mTOR Activation by Semliki Forest and Chikungunya Viruses Is Dependent on nsP3 and Connected to Replication Complex Internalization. J. Virol. 89, 11420-11437 (2015).

79. Cheng, F. et al. The Packaging of Different Cargo into Enveloped Viral Nanoparticles. Mol. Pharm. 10, 51-58 (2013).

80. Garmashova, N. et al. Analysis of Venezuelan Equine Encephalitis Virus Capsid Protein Function in the Inhibition of Cellular Transcription. J. Virol. 81, 13552-13565 (2007).

81. Atasheva, S., Fish, A., Fornerod, M. \& Frolova, E. I. Venezuelan Equine Encephalitis Virus Capsid Protein Forms a Tetrameric Complex with CRM1 and Importin $\alpha / \beta$ That Obstructs Nuclear Pore Complex Function. J. Virol. 84, 4158-4171 (2010).

82. Robert E. Johnston, C. J. P. Fields Virology. vol. 1 (2018).

83. Garoff, H., Sjöberg, M. \& Cheng, R. H. Budding of alphaviruses. Virus Res. 106, 103-116 (2004).

84. Brown, R. S., Wan, J. J. \& Kielian, M. The Alphavirus Exit Pathway: What We Know and What We Wish We Knew. Viruses 10, 89 (2018).

85. Rupp, J. C., Sokoloski, K. J., Gebhart, N. N. \& Hardy, R. W. Alphavirus RNA synthesis and non-structural protein functions. J. Gen. Virol. 96, 2483-2500 (2015).

86. Li, C. et al. mRNA Capping by Venezuelan Equine Encephalitis Virus nsP1: Functional Characterization and Implications for Antiviral Research. J. Virol. 89, 8292-8303 (2015).

87. Decroly, E., Ferron, F., Lescar, J. \& Canard, B. Conventional and unconventional mechanisms for capping viral mRNA. Nat. Rev. Microbiol. 10, 51-65 (2012).

88. Vasiljeva, L., Merits, A., Auvinen, P. \& Kääriäinen, L. Identification of a novel function of the alphavirus capping apparatus. RNA 5'-triphosphatase activity of Nsp2. J. Biol. Chem. 275, 17281-17287 (2000).

89. Ahola, T., Lampio, A., Auvinen, P. \& Kääriäinen, L. Semliki Forest virus mRNA capping enzyme requires association with anionic membrane phospholipids for activity. EMBO J. 18, 3164-3172 (1999).

90. Spuul, P. et al. Role of the Amphipathic Peptide of Semliki Forest Virus Replicase Protein nsP1 in Membrane Association and Virus Replication. J. Virol. 81, 872-883 (2007).

91. Kujala, P. et al. Biogenesis of the Semliki Forest Virus RNA Replication Complex. J. Virol. 75, 3873-3884 (2001).

92. Jones, R., Bragagnolo, G., Arranz, R. \& Reguera, J. Capping pores of alphavirus nsP1 gate membranous viral replication factories. Nature 1-5 (2020) doi:10.1038/s41586-020-30368.

93. Akhrymuk, I., Lukash, T., Frolov, I. \& Frolova, E. I. Novel Mutations in nsP2 Abolish Chikungunya Virus-Induced Transcriptional Shutoff and Make the Virus Less Cytopathic without Affecting Its Replication Rates. J. Virol. 93, e02062-18, /jvi/93/4/JVI.0206218.atom (2018).

94. Bhalla, N. et al. Host translation shutoff mediated by non-structural protein 2 is a critical factor in the antiviral state resistance of Venezuelan equine encephalitis virus. Virology 496, 147-165 (2016).

95. Kim, D. Y., Atasheva, S., Frolova, E. I. \& Frolov, I. Venezuelan Equine Encephalitis Virus nsP2 Protein Regulates Packaging of the Viral Genome into Infectious Virions. J. Virol. 87, 42024213 (2013). 
96. Gomez de Cedrón, M., Ehsani, N., Mikkola, M. L., García, J. A. \& Kääriäinen, L. RNA helicase activity of Semliki Forest virus replicase protein NSP2. FEBS Lett. 448, 19-22 (1999).

97. Das, P. K., Merits, A. \& Lulla, A. Functional cross-talk between distant domains of chikungunya virus non-structural protein 2 is decisive for its RNA-modulating activity. $J$. Biol. Chem. 289, 5635-5653 (2014).

98. Law, Y.-S. et al. Structural insights into RNA recognition by the Chikungunya virus nsP2 helicase. Proc. Natl. Acad. Sci. 116, 9558-9567 (2019).

99. Atasheva, S., Gorchakov, R., English, R., Frolov, I. \& Frolova, E. Development of Sindbis Viruses Encoding nsP2/GFP Chimeric Proteins and Their Application for Studying nsP2 Functioning. J. Virol. 81, 5046-5057 (2007).

100. Hahn, Y. S., Strauss, E. G. \& Strauss, J. H. Mapping of RNA-temperature-sensitive mutants of Sindbis virus: assignment of complementation groups $A, B$, and $G$ to nonstructural proteins. J. Virol. 63, 3142-3150 (1989).

101. Hardy, W. R. \& Strauss, J. H. Processing the nonstructural polyproteins of sindbis virus: nonstructural proteinase is in the C-terminal half of $\mathrm{nsP} 2$ and functions both in cis and in trans. J. Virol. 63, 4653-4664 (1989).

102. Russo, A. T., White, M. A. \& Watowich, S. J. The Crystal Structure of the Venezuelan Equine Encephalitis Alphavirus nsP2 Protease. Structure 14, 1449-1458 (2006).

103. Kim, K. H., Rümenapf, T., Strauss, E. G. \& Strauss, J. H. Regulation of Semliki Forest virus RNA replication: a model for the control of alphavirus pathogenesis in invertebrate hosts. Virology 323, 153-163 (2004).

104. Morazzani, E. M. et al. Proteolytic cleavage of host proteins by the Group IV viral proteases of Venezuelan equine encephalitis virus and Zika virus. Antiviral Res. 164, 106-122 (2019).

105. Göertz, G. P. et al. The Methyltransferase-Like Domain of Chikungunya Virus nsP2 Inhibits the Interferon Response by Promoting the Nuclear Export of STAT1. J. Virol. 92, e01008-18, /jvi/92/17/e01008-18.atom (2018).

106. LaStarza, M. W., Lemm, J. A. \& Rice, C. M. Genetic analysis of the nsP3 region of Sindbis virus: evidence for roles in minus-strand and subgenomic RNA synthesis. J. Virol. 68, 57815791 (1994).

107. Malet, H. et al. The Crystal Structures of Chikungunya and Venezuelan Equine Encephalitis Virus nsP3 Macro Domains Define a Conserved Adenosine Binding Pocket. J. Virol. 83, 6534-6545 (2009).

108. Eckei, L. et al. The conserved macrodomains of the non-structural proteins of Chikungunya virus and other pathogenic positive strand RNA viruses function as mono-ADPribosylhydrolases. Sci. Rep. 7, 1-18 (2017).

109. Abraham, R. et al. ADP-ribosyl-binding and hydrolase activities of the alphavirus nsP3 macrodomain are critical for initiation of virus replication. Proc. Natl. Acad. Sci. 115, E10457-E10466 (2018).

110. Abraham, R. et al. Both ADP-Ribosyl-Binding and Hydrolase Activities of the Alphavirus nsP3 Macrodomain Affect Neurovirulence in Mice. mBio 11, e03253-19, /mbio/11/1/mBio.03253-19.atom (2020).

111. Gao, Y., Goonawardane, N., Ward, J., Tuplin, A. \& Harris, M. Multiple roles of the nonstructural protein 3 (nsP3) alphavirus unique domain (AUD) during Chikungunya virus genome replication and transcription. PLOS Pathog. 15, e1007239 (2019).

112. Oberste, M. S., Parker, M. D. \& Smith, J. F. Complete Sequence of Venezuelan Equine Encephalitis Virus Subtype IE Reveals Conserved and Hypervariable Domains within the C Terminus of nsP3. Virology 219, 314-320 (1996). 
113. Foy, N. J., Akhrymuk, M., Shustov, A. V., Frolova, E. I. \& Frolov, I. Hypervariable domain of nonstructural protein nsP3 of Venezuelan equine encephalitis virus determines cellspecific mode of virus replication. J. Virol. 87, 7569-7584 (2013).

114. Aaskov, J., Jones, A., Choi, W., Lowry, K. \& Stewart, E. Lineage replacement accompanying duplication and rapid fixation of an RNA element in the nsP3 gene in a species of alphavirus. Virology 410, 353-359 (2011).

115. Foy, N. J. et al. Hypervariable domains of nsP3 proteins of New World and Old World alphaviruses mediate formation of distinct, virus-specific protein complexes. J. Virol. 87, 1997-2010 (2013).

116. Kim, D. Y. et al. New World and Old World Alphaviruses Have Evolved to Exploit Different Components of Stress Granules, FXR and G3BP Proteins, for Assembly of Viral Replication Complexes. PLoS Pathog. 12, (2016).

117. Montero, H. \& Trujillo-Alonso, V. Stress Granules in the Viral Replication Cycle. Viruses 3, 2328-2338 (2011).

118. Viral modulation of stress granules | Elsevier Enhanced Reader. https://reader.elsevier.com/reader/sd/pii/S0168170212002018?token=8AE08260178582E 32BDE2C8B2F2C868D9B7837DF0059A5F298BCF5FABC982B814224762A83353501A86570 9B68623749 doi:10.1016/j.virusres.2012.06.004.

119. Frolov, I., Kim, D. Y., Akhrymuk, M., Mobley, J. A. \& Frolova, E. I. Hypervariable Domain of Eastern Equine Encephalitis Virus nsP3 Redundantly Utilizes Multiple Cellular Proteins for Replication Complex Assembly. J. Virol. 91, (2017).

120. Götte, B., Utt, A., Fragkoudis, R., Merits, A. \& Mclnerney, G. M. Sensitivity of Alphaviruses to G3BP Deletion Correlates with Efficiency of Replicase Polyprotein Processing. J. Virol. 94, (2020).

121. Meshram, C. D. et al. Mutations in Hypervariable Domain of Venezuelan Equine Encephalitis Virus nsP3 Protein Differentially Affect Viral Replication. J. Virol. 94, (2020).

122. Li, G. P. \& Rice, C. M. Mutagenesis of the in-frame opal termination codon preceding nsP4 of Sindbis virus: studies of translational readthrough and its effect on virus replication. J. Virol. 63, 1326-1337 (1989).

123. de Groot, R. J., Rümenapf, T., Kuhn, R. J., Strauss, E. G. \& Strauss, J. H. Sindbis virus RNA polymerase is degraded by the N-end rule pathway. Proc. Natl. Acad. Sci. U. S. A. 88, 89678971 (1991).

124. Rubach, J. K. et al. Characterization of purified Sindbis Virus nsP4 RNA-dependent RNA Polymerase activity in vitro. Virology 384, 201-208 (2009).

125. Tomar, S., Hardy, R. W., Smith, J. L. \& Kuhn, R. J. Catalytic Core of Alphavirus Nonstructural Protein nsP4 Possesses Terminal Adenylyltransferase Activity. J. Virol. 80, 9962-9969 (2006).

126. Sreejith, R. et al. Mapping interactions of Chikungunya virus nonstructural proteins. Virus Res. 169, 231-236 (2012).

127. Chung, D.-H. et al. Discovery of a Novel Compound with Anti-Venezuelan Equine Encephalitis Virus Activity That Targets the Nonstructural Protein 2. PLOS Pathog. 10, e1004213 (2014).

128. Rupp, J. C., Jundt, N. \& Hardy, R. W. Requirement for the amino-terminal domain of sindbis virus nsP4 during virus infection. J Virol 85, 3449-60 (2011).

129. Valiente-Echeverría, F., Melnychuk, L. \& Mouland, A. J. Viral modulation of stress granules. Virus Res. 169, 430-437 (2012).

130. Strauss, J. H. \& Strauss, E. G. The alphaviruses: gene expression, replication, and evolution. Microbiol Rev 58, 491-562 (1994). 
131. Markland, W., McQuaid, T. J., Jain, J. \& Kwong, A. D. Broad-Spectrum Antiviral Activity of the IMP Dehydrogenase Inhibitor VX-497: a Comparison with Ribavirin and Demonstration of Antiviral Additivity with Alpha Interferon. Antimicrob. Agents Chemother. 44, 859-866 (2000).

132. Lukaszewski, R. A. \& Brooks, T. J. G. Pegylated Alpha Interferon Is an Effective Treatment for Virulent Venezuelan Equine Encephalitis Virus and Has Profound Effects on the Host Immune Response to Infection. J. Virol. 74, 5006-5015 (2000).

133. Raison, C. L., Demetrashvili, M., Capuron, L. \& Miller, A. H. Neuropsychiatric Adverse Effects of Interferon- $\alpha$. CNS Drugs 19, 105-123 (2005).

134. Furuta, Y. et al. Favipiravir (T-705), a novel viral RNA polymerase inhibitor. Antiviral Res. 100, (2013).

135. Julander, J. G., Smee, D. F., Morrey, J. D. \& Furuta, Y. Effect of T-705 treatment on western equine encephalitis in a mouse model. Antiviral Res. 82, 169-171 (2009).

136. Abdelnabi, R., Jochmans, D., Verbeken, E., Neyts, J. \& Delang, L. Antiviral treatment efficiently inhibits chikungunya virus infection in the joints of mice during the acute but not during the chronic phase of the infection. Antiviral Res. 149, 113-117 (2018).

137. Delang, L., Abdelnabi, R. \& Neyts, J. Favipiravir as a potential countermeasure against neglected and emerging RNA viruses. Antivir. Res 153, 85-94 (2018).

138. Gardner, C. L. et al. Antibody Preparations from Human Transchromosomic Cows Exhibit Prophylactic and Therapeutic Efficacy against Venezuelan Equine Encephalitis Virus. J. Virol. 91, (2017).

139. DeBono, A. et al. Novel RU486 (mifepristone) analogues with increased activity against Venezuelan Equine Encephalitis Virus but reduced progesterone receptor antagonistic activity. Sci. Rep. 9, 1-19 (2019).

140. Ahmed, A. et al. Human cathelicidin peptide LL-37 as a therapeutic antiviral targeting Venezuelan equine encephalitis virus infections. Antiviral Res. 164, 61-69 (2019).

141. Meister, G. et al. Human Argonaute2 Mediates RNA Cleavage Targeted by miRNAs and siRNAs. Mol. Cell 15, 185-197 (2004).

142. Collart, F. R. \& Huberman, E. Cloning and sequence analysis of the human and Chinese hamster inosine-5'-monophosphate dehydrogenase cDNAs. J. Biol. Chem. 263, 1576915772 (1988).

143. Madsen, C. et al. Small molecule inhibitors of Ago2 decrease Venezuelan equine encephalitis virus replication. Antiviral Res. 112, 26-37 (2014).

144. Urakova, N. et al. $\beta$-d-N4-Hydroxycytidine Is a Potent Anti-alphavirus Compound That Induces a High Level of Mutations in the Viral Genome. J. Virol. 92, (2018).

145. Chung, D. H. et al. HTS-Driven Discovery of New Chemotypes with West Nile Virus Inhibitory Activity. Molecules 15, 1690-1704 (2010).

146. Chung, D.-H. et al. Discovery of a Broad-Spectrum Antiviral Compound That Inhibits Pyrimidine Biosynthesis and Establishes a Type 1 Interferon-Independent Antiviral State. Antimicrob. Agents Chemother. 60, 4552-4562 (2016).

147. Adcock, R. S., Chu, Y.-K., Golden, J. E. \& Chung, D.-H. Evaluation of anti-Zika virus activities of broad-spectrum antivirals and NIH clinical collection compounds using a cell-based, high-throughput screen assay. Antiviral Res. 138, 47-56 (2017).

148. Chung, D.-H. et al. Discovery of a Novel Compound with Anti-Venezuelan Equine Encephalitis Virus Activity That Targets the Nonstructural Protein 2. PLOS Pathog. 10, e1004213 (2014).

149. Růžek, L. E., Radim Nencka, Erik de Clercq, Katherine Seley-Radtke, Daniel. Nucleoside analogs as a rich source of antiviral agents active against arthropod-borne flaviviruses - 
Luděk Eyer, Radim Nencka, Erik de Clercq, Katherine Seley-Radtke, Daniel Růžek, 2018. Antivir. Chem. Chemother. (2018).

150. Afdhal, N. et al. Ledipasvir and sofosbuvir for untreated HCV genotype 1 infection. $N$ Engl J Med 370, 1889-98 (2014).

151. Chen, F. et al. Efficacy and safety of nucleoside antiviral drugs for treatment of recurrent herpes labialis: a systematic review and meta-analysis. J. Oral Pathol. Med. 46, 561-568 (2017).

152. Mehta, S., Moore, R. D. \& Graham, N. M. H. Potential factors affecting adherence with HIV therapy. AIDS 11, 1665-1670 (1997).

153. Feng, J. Y. Addressing the selectivity and toxicity of antiviral nucleosides - Joy Y Feng, 2018. Antivir. Chem. Chemother. (2018).

154. Forrester, N. L. et al. Genome-scale phylogeny of the alphavirus genus suggests a marine origin. J Virol 86, 2729-38 (2012).

155. Urakova, N. et al. beta-D-N(4)-hydroxycytidine is a potent anti-alphavirus compound that induces high level of mutations in viral genome. J Virol (2017) doi:10.1128/JVI.01965-17.

156. Julander, J. G. et al. Treatment of Venezuelan equine encephalitis virus infection with (-)carbodine. Antivir. Res 80, 309-15 (2008).

157. Abdelnabi, R., Jochmans, D., Verbeken, E., Neyts, J. \& Delang, L. Antiviral treatment efficiently inhibits chikungunya virus infection in the joints of mice during the acute but not during the chronic phase of the infection. Antiviral Res. 149, 113-117 (2018).

158. Dong Hoon Chung. A Cell-Based Confirmatory Screen for Compounds that Inhibit VEEV, TC83. (2011).

159. Schroeder, C. E. et al. Development of (E)-2-((1,4-Dimethylpiperazin-2-ylidene)amino)-5nitro-N-phenylbenzamide, ML336: Novel 2-Amidinophenylbenzamides as Potent Inhibitors of Venezuelan Equine Encephalitis Virus. J. Med. Chem. 57, 8608-8621 (2014).

160. Lemm, J. A. \& Rice, C. M. Assembly of functional Sindbis virus RNA replication complexes: requirement for coexpression of P123 and P34. J. Virol. 67, 1905-1915 (1993).

161. Jing-Hsuing Ou, C. M. R. Sequence Studies of Several Alphavirus Genomic RNAs in the Region Containing the Start of the Subgenomic RNA. Proc. Natl. Acad. Sci. 79, 5235-5239 (2018).

162. Rikkonen, M., Peranen, J. \& Kaariainen, L. ATPase and GTPase activities associated with Semliki Forest virus nonstructural protein nsP2. J Virol 68, 5804-10 (1994).

163. Rupp, J. C., Jundt, N. \& Hardy, R. W. Requirement for the amino-terminal domain of sindbis virus nsP4 during virus infection. J. Virol. 85, 3449-3460 (2011).

164. John Aris. Fluorography of Gels Using PPO.

165. Plaskon, N. E., Adelman, Z. N. \& Myles, K. M. Accurate strand-specific quantification of viral RNA. PLoS One 4, e7468 (2009).

166. Barton, D. J., Sawicki, S. G. \& Sawicki, D. L. Solubilization and immunoprecipitation of alphavirus replication complexes. J. Virol. 65, 1496-1506 (1991).

167. Schindelin, J. et al. Fiji: an open-source platform for biological-image analysis. Nat Methods 9, 676-82 (2012).

168. Sawicki, D. L. \& Sawicki, S. G. Short-lived minus-strand polymerase for Semliki Forest virus. J. Virol. 34, 108-118 (1980).

169. Schneider-Poetsch, T. et al. Inhibition of eukaryotic translation elongation by cycloheximide and lactimidomycin. Nat Chem Biol 6, 209-217 (2010).

170. Albulescu, I. C., Tas, A., Scholte, F. E. M., Snijder, E. J. \& van Hemert, M. J. An in vitro assay to study chikungunya virus RNA synthesis and the mode of action of inhibitors. J. Gen. Virol. 95, 2683-2692 (2014). 
171. Roy, A., Kucukural, A. \& Zhang, Y. I-TASSER: a unified platform for automated protein structure and function prediction. Nat Protoc 5, 725-38 (2010).

172. Yang, J. et al. The I-TASSER Suite: protein structure and function prediction. Nat Methods 12, 7-8 (2015).

173. Zhang, Y. I-TASSER server for protein 3D structure prediction. BMC Bioinformatics $\mathbf{9}, 40$ (2008).

174. Francis, D. M. \& Page, R. Strategies to Optimize Protein Expression in E. coli. Curr. Protoc. Protein Sci. 61, 5.24.1-5.24.29 (2010).

175. Stewart, E. J., Åslund, F. \& Beckwith, J. Disulfide bond formation in the Escherichia coli cytoplasm: an in vivo role reversal for the thioredoxins. EMBO J. 17, 5543-5550 (1998).

176. Williams, D., Van Frank, R., Muth, W. \& Burnett, J. Cytoplasmic inclusion bodies in Escherichia coli producing biosynthetic human insulin proteins. Science $\mathbf{2 1 5}$, 687-689 (1982).

177. Oberg, K., Chrunyk, B. A., Wetzel, R. \& Fink, A. L. Native-like Secondary Structure in Interleukin-1.beta. Inclusion Bodies by Attenuated Total Reflectance FTIR. Biochemistry 33, 2628-2634 (1994).

178. Makrynitsa, G. I. et al. NMR study of non-structural proteins-part II: 1H, 13C, 15N backbone and side-chain resonance assignment of macro domain from Venezuelan equine encephalitis virus (VEEV). Biomol. NMR Assign. 9, 247-251 (2015).

179. Fros, J. J., Maten, E. van der, Vlak, J. M. \& Pijlman, G. P. The C-Terminal Domain of Chikungunya Virus nsP2 Independently Governs Viral RNA Replication, Cytopathicity, and Inhibition of Interferon Signaling. J. Virol. 87, 10394-10400 (2013).

180. Best, M. D. Click Chemistry and Bioorthogonal Reactions: Unprecedented Selectivity in the Labeling of Biological Molecules. Biochemistry 48, 6571-6584 (2009).

181. Mateos-Gil, P., Letschert, S., Doose, S. \& Sauer, M. Super-Resolution Imaging of Plasma Membrane Proteins with Click Chemistry. Front. Cell Dev. Biol. 4, (2016).

182. Ma, Y., McClatchy, D. B., Barkallah, S., Wood, W. W. \& Yates, J. R. Quantitative analysis of newly synthesized proteins. Nat. Protoc. 13, 1744-1762 (2018).

183. Eichelbaum, K. \& Krijgsveld, J. Rapid Temporal Dynamics of Transcription, Protein Synthesis, and Secretion during Macrophage Activation*,. Mol. Cell. Proteomics 13, 792810 (2014).

184. Marks, I. S. et al. Strain-Promoted "Click" Chemistry for Terminal Labeling of DNA. Bioconjug. Chem. 22, 1259-1263 (2011).

185. Stuhtmann, G. et al. lodixanol density gradient centrifugation for selecting stallion sperm for cold storage and cryopreservation. Anim. Reprod. Sci. 133, 184-190 (2012).

186. Law, Y.-S. et al. Structural insights into RNA recognition by the Chikungunya virus nsP2 helicase. Proc. Natl. Acad. Sci. 116, 9558-9567 (2019).

187. Pietilä, M. K., van Hemert, M. J. \& Ahola, T. Purification of Highly Active Alphavirus Replication Complexes Demonstrates Altered Fractionation of Multiple Cellular Membranes. J. Virol. 92, (2018).

188. Beck, C. E. \& Wyckoff, R. W. G. Venezuelan Equine Encephalomyelitis. Science 88, 530-530 (1938).

189. A Serological Difference Between Eastern and Western Equine Encephalomyelitis Virus Gael Ten Broeck, Malcolm H. Merrill, 1933. https://journals.sagepub.com/doi/abs/10.3181/00379727-31-7066C?journalCode=ebma.

190. Meyer, K. F., Haring, C. M. \& Howitt, B. The Etiology of Epizootic Encephalomyelitis of Horses in the San Joaquin Valley, 1930. Science 74, 227-228 (1931). 
191. Poirier, E. Z. et al. Low-Fidelity Polymerases of Alphaviruses Recombine at Higher Rates To Overproduce Defective Interfering Particles. J. Virol. 90, 2446-2454 (2016).

192. Bingham, R. J., Dykeman, E. C. \& Twarock, R. RNA Virus Evolution via a Quasispecies-Based Model Reveals a Drug Target with a High Barrier to Resistance. Viruses 9, 347 (2017).

193. Compton, J. R., Mickey, M. J., Hu, X., Marugan, J. J. \& Legler, P. M. Mutation of Asn-475 in the Venezuelan Equine Encephalitis Virus nsP2 Cysteine Protease Leads to a Self-Inhibited State. Biochemistry 56, 6221-6230 (2017).

194. Hu, X. et al. Kinetic, Mutational, and Structural Studies of the Venezuelan Equine Encephalitis Virus Nonstructural Protein 2 Cysteine Protease. Biochemistry 55, 3007-3019 (2016).

195. Karabatsos, N. \& Buckley, S. M. Susceptibility of the baby-hamster kidney-cell line (BHK-21) to infection with arboviruses. Am. J. Trop. Med. Hyg. 16, 99-105 (1967).

196. Isolation of a Sindbis virus variant by passage on mouse plasmacytoma cells. | Journal of Virology. https://jvi.asm.org/content/15/4/1037.

197. Sellers, R. F., Bergold, G. H., Suárez, O. M. \& Morales, A. Investigations During Venezuelan Equine Encephalitis Outbreaks in Venezuela-1962-1964. Am. J. Trop. Med. Hyg. 14, 460469 (1965).

198. Oie, H. K., Buckler, C. E., Uhlendorf, C. P., Hill, D. A. \& Baron, S. Improved assays for a variety of interferons. 1. Proc. Soc. Exp. Biol. Med. Soc. Exp. Biol. Med. N. Y. N 140, 11781181 (1972).

199. SH-SY5Y ATCC ${ }^{\circledR}$ CRL-2266 ${ }^{\text {TM }}$ Homo sapiens bone marrow neuroblast. https://www.atcc.org/products/all/CRL-2266.aspx\#documentation.

200. Shipley, M. M., Mangold, C. A., Kuny, C. V. \& Szpara, M. L. Differentiated Human SH-SY5Y Cells Provide a Reductionist Model of Herpes Simplex Virus 1 Neurotropism. J. Virol. 91, e00958-17, e00958-17 (2017).

201. Castorena, K. M., Peltier, D. C., Peng, W. \& Miller, D. J. Maturation-dependent responses of human neuronal cells to western equine encephalitis virus infection and type I interferons. Virology 372, 208-220 (2008).

202. Luplertlop, N. et al. The impact of Zika virus infection on human neuroblastoma (SH-SY5Y) cell line. J. Vector Borne Dis. 54, 207 (2017).

203. Encinas, M. et al. Sequential Treatment of SH-SY5Y Cells with Retinoic Acid and BrainDerived Neurotrophic Factor Gives Rise to Fully Differentiated, Neurotrophic FactorDependent, Human Neuron-Like Cells. J. Neurochem. 75, 991-1003 (2002).

204. Jämsä, A., Hasslund, K., Cowburn, R. F., Bäckström, A. \& Vasänge, M. The retinoic acid and brain-derived neurotrophic factor differentiated SH-SY5Y cell line as a model for Alzheimer's disease-like tau phosphorylation. Biochem. Biophys. Res. Commun. 319, 9931000 (2004).

205. Kovalevich, J. \& Langford, D. Considerations for the Use of SH-SY5Y Neuroblastoma Cells in Neurobiology. Methods Mol. Biol. Clifton NJ 1078, 9-21 (2013).

206. Dwane, S., Durack, E. \& Kiely, P. A. Optimising parameters for the differentiation of SHSY5Y cells to study cell adhesion and cell migration. BMC Res. Notes 6, 366 (2013).

207. Xicoy, H., Wieringa, B. \& Martens, G. J. M. The SH-SY5Y cell line in Parkinson's disease research: a systematic review. Mol. Neurodegener. 12, 10 (2017).

208. Shipley, M. M., Mangold, C. A. \& Szpara, M. L. Differentiation of the SH-SY5Y Human Neuroblastoma Cell Line. J. Vis. Exp. JoVE 53193 (2016) doi:10.3791/53193.

209. Robinson, J. T. et al. Integrative genomics viewer. Nat. Biotechnol. 29, 24-26 (2011).

210. Robinson, J. T., Thorvaldsdóttir, H., Wenger, A. M., Zehir, A. \& Mesirov, J. P. Variant Review with the Integrative Genomics Viewer. Cancer Res. 77, e31-e34 (2017). 
211. Thorvaldsdóttir, H., Robinson, J. T. \& Mesirov, J. P. Integrative Genomics Viewer (IGV): high-performance genomics data visualization and exploration. Brief. Bioinform. 14, 178192 (2013).

212. Cacciabue, M., Currá, A. \& Gismondi, M. I. ViralPlaque: a Fiji macro for automated assessment of viral plaque statistics. PeerJ 7, (2019).

213. Laemmli sample buffer (2X). Cold Spring Harb. Protoc. 2006, pdb.rec10424 (2006).

214. Kim, K. H., Rümenapf, T., Strauss, E. G. \& Strauss, J. H. Regulation of Semliki Forest virus RNA replication: a model for the control of alphavirus pathogenesis in ivertebrate hosts. Virology 323, 153-163 (2004).

215. Hahne, H. et al. Proteome Wide Purification and Identification of O-GIcNAc-Modified Proteins Using Click Chemistry and Mass Spectrometry. J. Proteome Res. 12, 927-936 (2013).

216. Das, P. K., Merits, A. \& Lulla, A. Functional cross-talk between distant domains of chikungunya virus non-structural protein 2 is decisive for its RNA-modulating activity. $J$. Biol. Chem. 289, 5635-5653 (2014).

217. Gomez de Cedrón, M., Ehsani, N., Mikkola, M. L., García, J. A. \& Kääriäinen, L. RNA helicase activity of Semliki Forest virus replicase protein NSP2. FEBS Lett. 448, 19-22 (1999).

218. Hahn, Y. S., Strauss, E. G. \& Strauss, J. H. Mapping of RNA- temperature-sensitive mutants of Sindbis virus: assignment of complementation groups $A, B$, and $G$ to nonstructural proteins. J. Virol. 63, 3142-3150 (1989).

219. Kim, K. H., Rümenapf, T., Strauss, E. G. \& Strauss, J. H. Regulation of Semliki Forest virus RNA replication: a model for the control of alphavirus pathogenesis in invertebrate hosts. Virology 323, 153-163 (2004).

220. SH-SY5Y ATCC ${ }^{\circledR}$ CRL-2266 ${ }^{\mathrm{TM}}$ Homo sapiens bone marrow neuroblast.

221. Kovalevich, J. \& Langford, D. Considerations for the Use of SH-SY5Y Neuroblastoma Cells in Neurobiology. Methods Mol. Biol. Clifton NJ 1078, 9-21 (2013). 
CURICULUM VITAE

Andrew Skidmore M.S

505 South Hancock Street 642 C

Louisville, Kentucky, 40202

(502)693-2328

amskid01@louisville.edu

\section{EDUCATION}

8/2011-5/2015 B.S. in Biology (suma cum laude), Bellarmine University, Louisville, Kentucky

8/2015-4/2017 M.S. in Microbiology and Immunology, University of Louisville, Louisville, Kentucky

8/2015-present Ph.D. in Microbiology and Immunology, University of Louisville, Louisville Kentucky

\section{ACADEMIC APPOINTMENTS}

8/2015-present Graduate research assistant, University of Louisville, Microbiology and Immunology, Lab of Dr. Dong-Hoon Chung

\section{PROFESSIONAL MEMBERSHIPS AND ACTIVITIES}

2016- Present Center of Predictive Medicine for Emerging Infectious Diseases (CPM) 2017- Present Student Member, American Society of Virology

2019-2020 Postgraduate Student Member, the Microbiology Society

\section{HONORS AND AWARDS}

2015-2017 Interdisciplinary Program in Biomedical Sciences Fellow, University of Louisville, Ky

7/2017 American Society of Virology graduate student travel award 7/2018 American Society of Virology graduate student travel award 5/2019 Graduate Student Council Travel Award University of Louisville 8/2019-Present Trainee T32 inflammation and pathogenesis training grant

\section{COMMITTEE ASSIGNMENTS AND ADMINISTRATIVE SERVICES}

2016-2019 Graduate Student Council

2016- 2019 Microbiology and immunology Student Organization, graduate student council representative 2017-2018 Senator at large, Graduate Student Council 2018-2019 University of Louisville Graduate Student Council Director of Travel 2017-2018 Organizer of University of Louisville virology discussion group

\section{ABSTRACTS AND PRESENTATIONS}




\section{ORAL PRESENTATIONS}

Local/Regional Meetings

1. Torsional Strength of Animal Long Bones, Honors thesis presentations, Bellarmine University, 4/2015, Louisville Ky

2. ML336 Inhibits Viral RNA Production of VEEV, Center for Predictive Medicine retreat, 10/2016, Starlight Indiana

3. nsP2 and RNA Production in VEEV, Virology Discussion Group, University of Louisville, 12/2016, Louisville Ky

4. A Novel Compound, ML336, Inhibits VEEV Replication by Interfering with Viral RNA Synthesis, department of Microbiology and Immunology seminar series, University of Louisville, 11/7/2017, Louisville Ky

5. Determination of the Biological Functions of Undefined Domains of VEEV nsP2 and nsP4, department of Microbiology and Immunology seminar series, University of Louisville, 4/9/19, Louisville Ky

National Meetings

1. A Novel Antiviral Compound, ML336, Inhibits VEEV Replication by Interfering with Viral RNA Synthesis, American Society of Virology, University of Maryland, College Park Ma. 7/17/2018

International Meetings

1. The molecular interaction between ML336 and the VEEV non-structural proteins. The annual meeting of the Microbiology Society, Edinburgh Scotland, 4/2020. Accepted for presentation, meeting cancelled.

\section{POSTERS}

Local/Regional Meetings

1. Torsional Strength of Animal Long Bones, Andrew M. Skidmore, David Porta, Butler undergraduate research conference, Butler University, Indianapolis Indiana, 4/2015

2. A Novel Compound, ML336, Inhibits RNA synthesis of VEEV, Andrew M. Skidmore, Robert S. Adcock, Colleen B. Jonsson, Jennifer E. Golden, and Dong-Hoon Chung, Research Louisville, University of Louisville, Louisville Ky, 10/2017

3. A Novel Compound, ML336, Inhibits RNA synthesis of VEEV, Andrew M. Skidmore, Robert S. Adcock, Colleen B. Jonsson, Jennifer E. Golden, and Dong-Hoon Chung, Research Louisville, University of Louisville, Louisville Ky, $10 / 2018$

4. The molecular activities of ML336 on the VEEV non-structural proteins. Andrew Skidmore, Dong-Hoon Chung, Research Louisville, University of Louisville, Louisville Ky, 10/2019

National Meetings

1. ML336, a Compound Inhibits RNA Synthesis of VEEV, Andrew Skidmore, Robert S. Adcock, Colleen B. Jonsson, Jennifer E. Golden, and Dong-Hoon Chung, American Society of Virology, University of Wisconsin Madison, Madison Wi, 6/2017 
2. The molecular interaction between ML336 and the VEEV non-structural proteins, Andrew M. Skidmore, Robert S. Adcock, Jennifer E. Golden, and Dong-Hoon Chung, American Society of Virology, University of Minnesota, Minneapolis Mn, $7 / 2019$

International Meetings

1. A Novel Compound, ML336, Inhibits VEEV Replication by Interfering with Viral RNA Synthesis, Andrew M. Skidmore, Robert S. Adcock, Jasper Lee, Colleen B. Jonsson, Jennifer E. Golden, Dong-Hoon Chung, International Congress of Antiviral Research, Porto Portugal, 6/2018. Presented by Dong-Hoon Chung.

\section{PUBLICATIONS}

\section{PEER-REVIEWED ORIGINAL RESEARCH MANUSCRIPTS}

1. Rapid Sequencing of Multiple RNA Viruses in Their Native Form, Wongsurawat T, Jenjaroenpun P, Taylor MK, Lee J, Tolardo AL, Parvathareddy J, Kandel S, Wadley TD, Kaewnapan B, Athipanyasilp N, Skidmore A, Chung D, Chaimayo C, Whitt M, Kantakamalakul W, Sutthent R, Horthongkham N, Ussery DW, Jonsson CB, Nookaew I., Frontiers in Microbiology, 2/25/2019

2. Benzamidine ML336 inhibits plus and minus strand RNA synthesis of Venezuelan equine encephalitis virus without affecting host RNA production. Andrew Skidmore, Robert Adcock, Colleen Jonsson, Jennifer Golden, Donghoon Chung, Antiviral Research, 12/6/2019

\section{TEACHING AND RELATED ACTIVITIES}

\section{LECTURES}

1. RNA isolation and analysis, section for Methods in Biomedical Science for the Department of Microbiology and Immunology, University of Louisville, given 2017-2020.

2. Viral Transmission, section for Introduction to Virology, University of Louisville, given 2020 and 2021.

\section{Training}

1. Summer 2018 STEM Graduate Teaching Assistant Academy, 7/23/2018-

$7 / 27 / 2018$. A condensed training for graduate students in the STEM disciplines interested in teaching. University of Louisville, hosted by the Graduate School. 